\title{
Separating positivity and regularity for fourth order Dirichlet problems in 2d-domains
}

\section{Anna Dall'Acqua, Christian Meister* and Guido Sweers}

Summary The main result in this paper is that the solution operator for the bi-laplace problem with zero Dirichlet boundary conditions on a bounded smooth $2 \mathrm{~d}$-domain can be split in a positive part and a possibly negative part which both satisfy the zero boundary condition. Moreover, the positive part contains the singularity and the negative part inherits the full regularity of the boundary. Such a splitting allows one to find a priori estimates for fourth order problems similar to the one proved via the maximum principle in second order elliptic boundary value problems. The proof depends on a careful approximative fill-up of the domain by a finite collection of limaçons. The limaçons involved are such that the Green function for the Dirichlet bi-laplacian on each of these domains is strictly positive.

2000 Mathematics Subject Classification: Primary 35J30; Secondary 31A30; 35B50.

Key words: Biharmonic Operator, Dirichlet Boundary Conditions, Green function estimates, Positivity, Maximum Principle.

* Supported by N.W.O., the Netherlands Organisation for Scientific Research 


\section{Contents}

1 Introduction and main results $\mathbf{3}$

1.1 Main results . . . . . . . . . . . . . . . . . . . . 4

1.2 Some notations . . . . . . . . . . . . . . 6

2 Domains with a positive biharmonic Green function 9

2.1 Limaçon de Pascal . . . . . . . . . . . . . . . . . . . 9

2.2 Perturbations from the bilaplacian on a limaçon . . . . . . . . . . . 12

2.2.1 Conformal transformation ............... 14

2.2.2 Proof of the perturbation theorem ........... 18

3 An approximate filling of the domain by perturbed limaçons 20

3.1 Local approximation . . . . . . . . . . . . . . . 21

3.2 Approximation by a limaçon in one point . . . . . . . . . . . 24

3.3 Construction of the mapping $f_{a, R} \ldots \ldots \ldots 24$

3.4 The mapping is close to the identity in $C^{2, \gamma}$-sense . . . . . . . . . . 27

3.5 Bounded third and fourth derivative of the mapping . . . . . . . . . 28

3.6 The covering ......................... 29

4 Proving the estimates 30

4.1 A maximum principle type estimate . . . . . . . . . . . . . . 30

4.2 Green function estimates . . . . . . . . . . . . . . 39

A Improved $\varepsilon$-closeness to the disk 43

B Previously known estimates for $G_{\Omega_{a}} \quad 44$

C Technical lemmas

C.1 The mapping is close to identity . . . . . . . . . . . . . . 45

C.1.1 Proof of Proposition $3.10 \ldots \ldots$. . . . . . . . 45

C.1.2 Proof of Proposition $3.11 \ldots \ldots$. . . . . . . . . . 46

C.2 Bounded third and fourth derivative of the mapping . . . . . . . . 52

C.2.1 Proof of Proposition $3.12 \ldots \ldots \ldots 52$

C.2.2 Proof of Proposition $3.13 \ldots \ldots 53$

C.3 Partition of unity . . . . . . . . . . . . 60

D Elliptic regularity and interpolation $\quad 60$

D.1 Classical results . . . . . . . . . . . . . . . . . . 61

D.2 Regularity for strong solutions . . . . . . . . . . . . . 62

D.3 Regularity for weak solutions . . . . . . . . . . . . . . . . . 64

D.4 Regularity between weak and strong . . . . . . . . . . . . 65 


\section{Introduction and main results}

A major tool for second order elliptic equations is the maximum principle. The maximum principle not only implies that a positive source will give a positive solution but it helps to obtain a priori estimates and hence to find regularity results. Especially in nonlinear equations such a priori estimates play a crucial role. Several results are referred to by the name maximum principle but the result that we want to refer to is the local result that reads for the laplacian as $\Delta u \geq 0$ in a neighborhood of $a$ implies that $u$ cannot have a strict maximum in $a$. A serious obstruction for higher order elliptic equations is that one cannot expect a similar result as functions like $\pm x^{2}$ clearly show.

The situation becomes more complicated when considering a positivity preserving property which is often also named "maximum principle". For the laplacian that is: $-\Delta u \geq 0$ in $\Omega$ and $u \geq 0$ on $\partial \Omega$ implies $u \geq 0$ in $\Omega$ (with $\Omega$ a bounded domain in $\mathbb{R}^{n}$ ). This "global maximum principle" also holds for some special higher dimensional problems. Indeed, $\Delta^{2} u \geq 0$ in $B$ and $-\frac{\partial}{\partial|x|} u \geq 0, u \geq 0$ on $\partial B$ implies $u \geq 0$ in $B$. Here $B$ is a ball in $\mathbb{R}^{n}$ with $n \leq 4$. For this special result see [20, page 34] or [16]. With $\frac{\partial}{\partial|x|} u=u=0$ on $\partial B$ the result holds for $B$ in any $\mathbb{R}^{n}$ and goes back 100 years to Boggio ([3]). The restriction to the ball is rather crucial. Since Duffin's first counterexample ([10]) many others followed and it is conceivable that for most domains a positivity preserving property fails (see [15]).

In [19] Nehari looks for subdomains of $\Omega$, characterized by the position of the points $x$ and $y$ and by simple geometric properties of $\Omega$, in which the Green function for the biharmonic problem with Dirichlet boundary condition on $\Omega$ may be shown to be positive.

In order to find a priori estimates it is however not necessary to have such a sign preserving result; it is sufficient that one can show a uniform behavior of the singularity of the solution operator. A separation of the solution operator in a smooth but sign changing part and a uniform singular part of fixed sign is the main result of the present paper. Since we are using conformal mappings our present result is restricted to two dimensional domains. Note that in two dimensions the singularity of the solution operator for the bilaplacian appears in the second derivative. Indeed a fundamental solution is $\frac{1}{8 \pi}|x|^{2} \ln |x|$.

Let us be more precise. For $\Omega$ an open bounded $C^{4, \alpha}$ domain in $\mathbb{R}^{2}$ we 
will show that the solution operator for

$$
\left\{\begin{aligned}
\Delta^{2} u & =f \text { in } \Omega, \\
u & =0 \text { on } \partial \Omega, \\
\frac{\partial}{\partial \nu} u & =0 \text { on } \partial \Omega,
\end{aligned}\right.
$$

can be split in the way we just mentioned. Crucial is that we find a uniform behavior of such a splitting even near the boundary. Such a result away from the boundary, that is in compact subsets of $\Omega$, was proven in [14].

We proceed as follows. We recall from [7] that for $\Omega$ taken from some family of limaçons the Green function for (1.1) is positive. Secondly, one may show that small perturbations of those limaçons do not destroy the positivity of the corresponding Green function. Thirdly, one may construct a finite number of such slightly perturbed limaçons $\left\{E_{j} \subset \mathbb{R}^{2}\right\}$ that are such that the boundary of $\Omega$ is covered by the boundaries of those perturbed limaçons while these limaçons cover a neighborhood of the boundary of $\Omega$. Together with a covering of the interior one is able to construct the desired splitting of the solution through a partition of unity related to that covering. Roughly explained, for each $x \in \Omega$ there is an element $E_{j}$ in this finite covering such that the Green function for (1.1) can be decomposed as the sum of $G_{E_{j}}(x, y)$ and a remainder term $G_{j}^{\text {rest }}(x, y)$ where $G_{E_{j}}(x, y)$ is positive and $G_{j}^{\text {rest }}(x, y)$ is without singularity. Note that the choice of $E_{j}$ depends on $x$. Since the extension of $G_{E_{j}}(x, y)$ from $E_{j}^{2}$ to $\Omega^{2}$ by 0 is not smooth one may guess that the just mentioned decomposition is more involved than just this simple sum.

\subsection{Main results}

In this section we state the two main results of the paper. First we fix some notation.

The Green function $G_{\Omega}$ is such that the solution of problem (1.1) for appropriate $f$ can be written as

$$
u(x)=\int_{\Omega} G_{\Omega}(x, y) f(y) d y .
$$

In the following $d_{\Omega}($.$) denotes the distance to the boundary in the domain$ $\Omega$ :

$$
d_{\Omega}(z):=\inf _{x \in \partial \Omega}|z-x|
$$


Two closely related versions of the main result are the following. The first one is a pointwise description which focusses on the splitting of the solution operator.

Theorem 1.1 Assume that $\Omega \subset \mathbb{R}^{2}$ is a bounded simply connected domain with $\partial \Omega \in C^{16}$. Then there exist $G_{\Omega}^{\text {reg }}, G_{\Omega}^{\text {sing }}: \bar{\Omega}^{2} \rightarrow \mathbb{R}$ such that the Green function for (1.1) can be written as

$$
G_{\Omega}(x, y)=G_{\Omega}^{r e g}(x, y)+G_{\Omega}^{\text {sing }}(x, y)
$$

and the following is satisfied:

1. (a) $G_{\Omega}^{\text {sing }}(x, y) \geq 0$ on $\bar{\Omega}^{2}$;

(b) $G_{\Omega}^{\text {sing }} \in C^{1, \gamma}\left(\bar{\Omega}^{2}\right) \cap C_{0}^{1}\left(\bar{\Omega}^{2}\right)$ for all $\gamma \in(0,1)$;

(c) $G_{\Omega}^{\text {sing }} \in C^{15, \gamma}\left(\left\{(x, y) \in \bar{\Omega}^{2} ; x \neq y\right\}\right)$ for all $\gamma \in(0,1)$;

2. (a) $G_{\Omega}^{\text {reg }} \in C^{15, \gamma}\left(\bar{\Omega}^{2}\right) \cap C_{0}^{1}\left(\bar{\Omega}^{2}\right)$ for all $\gamma \in(0,1)$.

Remark 1.2 The space $C_{0}^{1}(K)$ consists of all functions $g \in C^{1}(K)$ such that $g=|D g|=0$ on $\partial K$.

Remark 1.3 For the condition $\partial \Omega \in C^{16}$ see Definition 1.11. We expect that $C^{16}$ can be relaxed. However, since we are using results from [18] we depend on the assumptions in that paper.

Remark 1.4 Since $G_{\Omega}$ is symmetric one may assume that both $G_{\Omega}^{\text {reg }}$ and $G_{\Omega}^{\text {sing }}$ are symmetric. Indeed, one may symmetrize by setting

$$
G_{\Omega, \text { new }}^{\cdots}(x, y):=\frac{1}{2} G_{\Omega}^{\cdots}(x, y)+\frac{1}{2} G_{\Omega}^{\cdots}(y, x) .
$$

In [8] it is shown that the Green function satisfies the following estimate for a two-dimensional domain $\Omega$ :

$$
\left|G_{\Omega}(x, y)\right| \leq c_{\Omega} d(x) d(y) \min \left\{1, \frac{d(x) d(y)}{|x-y|^{2}}\right\} \text { for every } x, y \in \Omega .
$$

By the estimates for the disk (see e.g. [12]) one finds that (1.2) is optimal for the positive part. Here we are able to get a better estimate from below: 
Theorem 1.5 Let $\Omega$ be a bounded domain in $\mathbb{R}^{2}$ with $\partial \Omega \in C^{16}$. Then there exists $c_{\Omega}>0$ such that $G_{\Omega}$ satisfies:

$$
G_{\Omega}(x, y) \geq-c_{\Omega} d(x)^{2} d(y)^{2} \text { for every } x, y \in \Omega .
$$

The next result is a kind of maximum principle, that is, it gives a pointwise bound from above for the solution in terms of the positive part of the right hand side and a weaker norm of the solution itself. Before we state the result let us recall that the space $W^{-m, p}(\Omega)$ is the dual space of $W_{0}^{m, p^{\prime}}(\Omega)$, with $\frac{1}{p}+\frac{1}{p^{\prime}}=1$, and its norm can be defined as follows

$$
\|u\|_{W^{-m, p}(\Omega)}:=\sup \left\{u(\varphi) ; \varphi \in W_{0}^{m, p^{\prime}}(\Omega),\|\varphi\|_{W^{m, p^{\prime}}(\Omega)} \leq 1\right\} .
$$

Theorem 1.6 Let $0<\alpha<1$ and $p \in(1, \infty)$. Suppose that $\Omega$ is a bounded simply connected domain in $\mathbb{R}^{2}$ with $\partial \Omega \in C^{4, \alpha}$. Then for any $q>2$ and $\varepsilon>0$ there exists a constant $c_{q, \Omega, \varepsilon}>0$ such that for $f \in L^{p}(\Omega)$ the solution $u \in W^{4, p}(\Omega) \cap W_{0}^{2, p}(\Omega)$ of (1.1) satisfies

$$
u(x) \leq c_{q, \Omega, \varepsilon}\left(\left\|f^{+}\right\|_{L^{1}(B(x, \varepsilon) \cap \Omega)}+\|u\|_{W^{-1, q}(\Omega)}\right) \text { for every } x \in \Omega .
$$

Here $f^{+}$denotes the positive part of $f$.

Remark 1.7 More precise information on how $c_{q, \Omega, \varepsilon}$ depends on $q, \Omega$ and $\varepsilon$ can be found in Theorem 4.1. For those who want to avoid norms for negative Sobolev spaces we recall that $\|u\|_{W^{-1, q}(\Omega)} \leq c(s, q, \Omega)\|u\|_{L^{s}(\Omega)}$ for $s \geq 2 q(q+2)^{-1}$.

\subsection{Some notations}

Let us fix the following (for later use we consider $\mathbb{R}^{n}$ with general $n$ ).

Notation 1.8 Let $\Omega$ be a bounded domain in $\mathbb{R}^{n}$ and let $f$ and $g$ be functions on $\Omega \times \Omega$.

- For $\alpha, \beta \in \mathbb{N}^{n}$ we set $|\alpha|=\sum_{k=1}^{n} \alpha_{k}$ and

$$
D_{x}^{\alpha} D_{y}^{\beta} f(x, y)=\frac{\partial^{|\alpha|}}{\partial x_{1}^{\alpha_{1}} x_{2}^{\alpha_{2}} . x_{n}^{\alpha_{n}}} \frac{\partial^{|\beta|}}{\partial y_{1}^{\beta_{1}} y_{2}^{\beta_{2}} . y_{n}^{\beta_{n}}} f(x, y) .
$$


- An equivalence relation for $f$ and $g$ which are nonnegative (See [13]):

$$
f \sim g \text { on } \Omega \times \Omega
$$

if and only if there are $c_{1}, c_{2}>0$ such that

$$
c_{1} f(x, y) \leq g(x, y) \leq c_{2} f(x, y) \text { for all } x, y \in \Omega
$$

- A dominance relation with respect to a nonnegative $f$ :

$$
f \preceq g \text { on } \Omega \times \Omega
$$

if and only if there is $c>0$ such that

$$
f(x, y) \leq c g(x, y) \text { for all } x, y \in \Omega
$$

The Hölder spaces $C^{r}(\bar{\Omega})$ and $C^{r, \gamma}(\bar{\Omega})$ with $r \in \mathbb{N}$ and $\gamma \in(0,1]$ are supplied with the norm:

$$
\begin{aligned}
\|f\|_{C^{r}(\bar{\Omega})} & :=\sum_{|\alpha| \leq r}\left\|D^{\alpha} f\right\|_{\infty}, \\
\|f\|_{C^{r, \gamma}(\bar{\Omega})} & :=\|f\|_{C^{r}(\bar{\Omega})}+\sum_{|\alpha|=r}\left[D^{\alpha} f\right]_{\gamma},
\end{aligned}
$$

where $[f]_{\gamma}:=\sup \left\{\frac{|f(x)-f(y)|}{|x-y|^{\gamma}} ; x, y \in \bar{\Omega}, x \neq y\right\}$. For convenience we set $C^{r, 0}(\bar{\Omega}):=C^{r}(\bar{\Omega})$. In the following $C_{c}^{r}(\Omega)$ denotes the set of all functions in $C^{r}(\Omega)$ whose supports are compact subsets of $\Omega$.

For $m \in \mathbb{N}$ and $p \geq 1, p \in \mathbb{R}, W^{m, p}(\Omega)$ denotes the Sobolev space with the norm

$$
\|f\|_{W^{m, p}(\Omega)}=\sum_{|\alpha| \leq m}\left\|D^{\alpha} f\right\|_{L^{p}(\Omega)} .
$$

We fix the following notation to point out on which quantities the constants depend.

Notation 1.9 For $\alpha, \beta, \gamma \in \mathbb{R}, C=C(\alpha, \beta, \gamma)$ means that $C$ depends only on $\alpha, \beta$ and $\gamma$, and that $C$ is bounded for bounded values of these parameters. 
Next we will need some notation concerning the domain and its boundary.

Notation 1.10 (relatively open subset of the boundary) For $K$ a subset of $\partial \Omega \subset \mathbb{R}^{n}$, set

$$
K^{\circ, \partial \Omega}:=\left(K \cup(\partial \Omega)^{c}\right)^{\circ} \cap \partial \Omega .
$$

In the literature several definitions of $C^{\ell, \alpha}$-domains appear. To avoid any ambiguity we explicitly give the version that we will use.

Definition 1.11 (uniform $C^{\ell, \alpha}$ regularity condition for $\Omega$ ) Let $\ell \in \mathbb{N}^{+}$, $\alpha \in[0,1]$ and $\Omega$ be a bounded domain in $\mathbb{R}^{n}$. The domain $\Omega$ satisfies the uniform $C^{\ell, \alpha}$ regularity condition (we write $\partial \Omega \in C^{\ell, \alpha}$ ) if there exist a positive constant $M$, a finite open covering $\left\{U_{j}\right\}_{j \in J}$ of $\partial \Omega$, a corresponding collection $\left\{\varphi_{j}\right\}_{j \in J}$ of $C^{\ell, \alpha}$ mappings such that for every $j \in J$ :

1. $\varphi_{j}: U_{j} \rightarrow B=\left\{y \in \mathbb{R}^{n}:|y|<1\right\}$ is a diffeomorphism; set $\psi_{j}=$ $\varphi_{j}^{i n v}$;

2. with $\left(\varphi_{j, 1}, \ldots, \varphi_{j, n}\right)$ and $\left(\psi_{j, 1}, \ldots, \psi_{j, n}\right)$ the components of $\varphi_{j}$ and $\psi_{j}$ :

$$
\left\|\varphi_{j, i}\right\|_{C^{\ell, \alpha}\left(\bar{U}_{j}\right)} \leq M \text { and }\left\|\psi_{j, i}\right\|_{C^{\ell, \alpha}(\bar{B})} \leq M \text { for all } i
$$

3. $\varphi_{j}\left(U_{j} \cap \Omega\right)=\left\{y \in B: y_{n}>0\right\}$;

and there exists $\delta>0$ such that

$$
\{x \in \Omega: d(x, \partial \Omega)<\delta\} \subset \bigcup_{j \in J} \psi_{j}\left(\left\{y \in \mathbb{R}^{n}:|y|<\frac{1}{2}\right\}\right) .
$$

Definition 1.11 is similar to the uniform $C^{\ell}$ regularity condition in [1, Def.4.10 page 84].

It will also be convenient to fix the following numbers.

Notation 1.12 Let $\Omega$ be a bounded domain with $\partial \Omega \in C^{2}$.

1. We write $\rho_{\Omega}$ for the largest number $r$ such that both $\Omega$ and $\mathbb{R}^{n} \backslash \Omega$ can be filled with balls of radius $r$. To be precise: for $r>0$ set $\Omega_{r}:=$ 
$\{z \in \Omega: d(z, \partial \Omega) \geq r\}, \tilde{\Omega}_{r}:=\left\{z \in \mathbb{R}^{n} \backslash \bar{\Omega}: d(z, \partial \Omega) \geq r\right\}$. Let
$\rho_{\Omega}>0$ be the largest $r$ such that the following holds:

$$
\Omega=\bigcup_{z \in \Omega_{r}} B_{r}(z) \text { and } \mathbb{R}^{n} \backslash \bar{\Omega}=\bigcup_{z \in \tilde{\Omega}_{r}} B_{r}(z) .
$$

2. We will also use $R_{\Omega}$ defined as the smallest $R$ such that $\Omega \subset B_{R}(z)$ for some $z \in \mathbb{R}^{2}$.

Remark 1.13 For most domains we may take $\rho_{\Omega}=\kappa^{-1}$ where $\kappa$ denotes the maximal curvature. But notice that $\rho_{\Omega}$ can be strictly smaller than $\kappa^{-1}$. For example this happens in the case of a dumb-bell shaped domain with a very narrow passage.

\section{Domains with a positive biharmonic Green function}

In this section we concentrate on the positivity preserving property of problem (1.1) in two-dimensional domain. Let us first settle what we mean by this.

Definition 2.1 We say that problem (1.1) on a domain $\Omega$ satisfies the positivity preserving property iffor any $f \geq 0$ the solution $u$ of (1.1) satisfies $u \geq 0$.

Obviously (1.1) on a domain $\Omega$ satisfies the positivity preserving property if and only if the biharmonic Green function associated to problem (1.1) on $\Omega$ is positive.

It is well known that problem (1.1) is positivity preserving on the disk (see [3]). In the following we first recall a recent result in [7] where a family of domains (limaçons) is given on which the biharmonic Green function associated to problem (1.1) is positive. Next we will show that small $C^{2, \gamma}$ perturbations of these domains do not destroy this property.

\subsection{Limaçon de Pascal}

In [7] one finds that on some limaçons the Green function for (1.1) is strictly positive. Since these limaçons are our starting point we will shortly recall some properties of these domains. 
The Limaçon de Pascal $\Omega_{a}$ with $a \in\left[0, \frac{1}{2}\right]$ is defined as the image of the unit disk through the conformal map

$$
h_{a}\left(x_{1}, x_{2}\right)=\left(x_{1}+2 a x_{1} x_{2}, x_{2}+a x_{2}^{2}-a x_{1}^{2}+1-a\right) .
$$

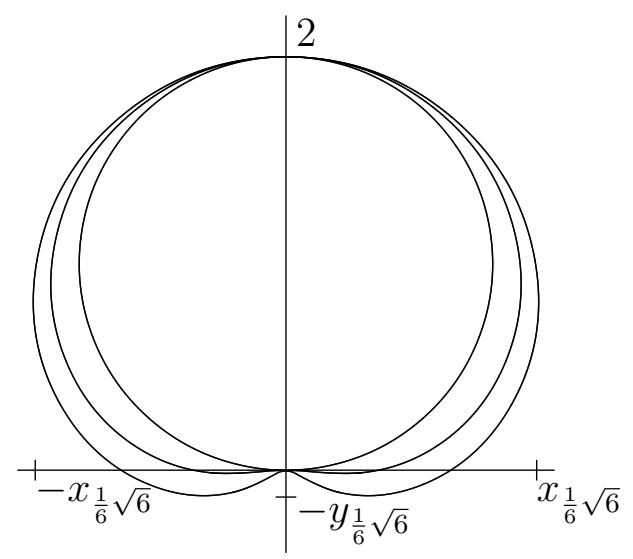

Figure 2.1 Limaçons $\Omega_{a}$ for respectively $a=0, a=\frac{3}{10}$ and $a=\frac{1}{6} \sqrt{6}$.

The result that is proved in [7] is the following:

Proposition 2.2 The Green function $G_{\Omega_{a}}$ for (1.1) with $\Omega=\Omega_{a}$ and $a \in$ $\left[0, \frac{1}{2}\right]$ is positive if and only if $a \in\left[0, \frac{1}{6} \sqrt{6}\right]$. Moreover, there exist $c_{1}, c_{2}>$ 0 such that for $a \in\left[0, \frac{1}{6} \sqrt{6}\right]$ the following estimates hold. Writing for short $d_{a}()=.d_{\Omega_{a}}($.$) :$

$$
\begin{aligned}
& G_{\Omega_{a}}(x, y) \leq c_{1} d_{a}(x) d_{a}(y) \min \left\{1, \frac{d_{a}(x) d_{a}(y)}{|x-y|^{2}}\right\}, \\
& G_{\Omega_{a}}(x, y) \geq c_{2}\left(\frac{1}{6} \sqrt{6}-a\right) d_{a}(x) d_{a}(y) \min \left\{1, \frac{d_{a}(x) d_{a}(y)}{|x-y|^{2}}\right\} .
\end{aligned}
$$

Remark 2.3 In [17] Hadamard was able to compute an explicit formula for the biharmonic Green function on a limaçon. The fact that this Green function is positive for $a \in\left[0, \frac{1}{6} \sqrt{6}\right]$ has been proven in [7].

We will also need scaled limaçons and we will define these for $R>0$ by

$$
\Omega_{a, R}:=\left\{(R x, R y):(x, y) \in h_{a}\left(B_{1}(0)\right)\right\},
$$


with $B_{1}(0)=\left\{(\eta, \xi) \in \mathbb{R}^{2}: \eta^{2}+\xi^{2}<1\right\}$. In the following $\Omega_{a}$ denotes $\Omega_{a, 1}$.

In the present paper we will consider limaçons $\Omega_{a, R}$ for $a \in[0, \bar{a}]$ where $\bar{a}$ is strictly between $\frac{1}{4}$ and $\frac{1}{6} \sqrt{6}$. By taking $\bar{a}$ strictly smaller than $\frac{1}{6} \sqrt{6}$ we will obtain estimates of the Green function $G_{\Omega_{a}}(.,$.$) which are$ uniform with respect to $a$.

\section{Some geometrical facts for the limaçon:}

1. For all $a \in\left[0, \frac{1}{2}\right]$ the limaçon $\Omega_{a, R}$ is symmetric with respect to the second axis and both $(0,0)$ and $(0,2 R)$ lie on $\partial \Omega_{a, R}$. Special values of the parameter $a$ are the following:

- $a=0: \Omega_{0, R}$ is the disk with radius $R$ and center $(0, R)$;

- $a=\frac{1}{4}: \Omega_{a, R}$ is convex if and only if $a \in\left[0, \frac{1}{4}\right]$;

- $a=\frac{1}{6} \sqrt{6} \approx .40825$ : the Green function associated to (1.1) for $\Omega=\Omega_{a, R}$ is positive if and only if $a \in\left[0, \frac{1}{6} \sqrt{6}\right]$, see [7];

- $a=\frac{1}{2}: \Omega_{\frac{1}{2}, R}$ is a cardioid.

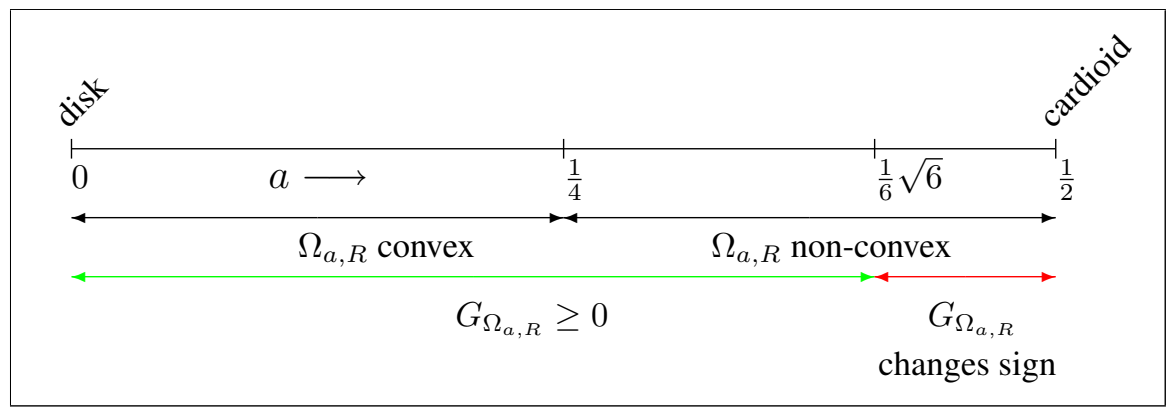

Figure 2.2 In the graph the critical values of the parameter a for convexity of the limaçons and positivity of the Green function.

2. Let $\left[-x_{a}, x_{a}\right] \times\left[-y_{a}, 2\right]$ denote the smallest rectangle that contains $\Omega_{a, 1}$. Then

$$
a \mapsto x_{a} \text { and } a \mapsto y_{a}
$$

are nondecreasing functions for $a \in\left[0, \frac{1}{2}\right]$ with $1 \leq x_{a} \leq 1.3$ and $0 \leq y_{a} \leq 0.25$. 
3. For $a \in\left[\frac{3}{16}, \frac{5}{16}\right]$, we will use $k_{a, R}:\left[-R x_{a}, R x_{a}\right] \rightarrow \mathbb{R}$ to describe the lower part of the boundary $\partial \Omega_{a, R}$ :

$$
k_{a, R}(x)=\inf \left\{y:(x, y) \in \Omega_{a, R}\right\} .
$$

In particular in the approximation we will use that the following relations hold:

$$
\begin{aligned}
& k_{a, R}^{\prime \prime}(0)= \frac{1}{R} \frac{1-4 a}{(1-2 a)^{2}} \text { and } \\
&\left\|\frac{\partial^{i}}{\partial x^{i}} k_{a, R}\right\|_{C^{0}\left[-R x_{a}^{*}, R x_{a}^{*}\right]} \leq \frac{b_{i}}{R^{i-1}} \text { for } i=1, \ldots, 5,
\end{aligned}
$$

with $x_{a}^{*}=\frac{1}{2}(1-\sqrt{3} a)$. Notice that $x_{a}^{*} \in\left(\frac{1}{5} x_{a}, \frac{1}{2} x_{a}\right)$ where $x_{a}$ is defined near (2.2). The constants $b_{i}$ can be taken independently of $a \in\left[\frac{3}{16}, \frac{5}{16}\right]$.

\subsection{Perturbations from the bilaplacian on a limaçon}

In this section we study the positivity preserving property of problem (1.1) on a domain $\Omega \subset \mathbb{R}^{2}$ that is $\varepsilon$-close in a $C^{2, \gamma}$-sense to a limaçon.

The concept of $\varepsilon$-closeness of domains that we use is the one introduced in [12, Def.1.1]. For sake of completeness we recall the definition.

Definition 2.4 Let $\varepsilon>0$. We call $\Omega \varepsilon$-close in $C^{k, \gamma}$-sense to $\Omega^{*}$ if there exists a $C^{k, \gamma}$-mapping $g: \bar{\Omega}^{*} \rightarrow \bar{\Omega}$ such that $g\left(\bar{\Omega}^{*}\right)=\bar{\Omega}$ and

$$
\|g-I d\|_{C^{k, \gamma}\left(\bar{\Omega}^{*}\right)} \leq \varepsilon .
$$

The main result of the section is the following.

Theorem 2.5 (Perturbation of the domain) Let $\bar{a} \in\left(\frac{1}{4}, \frac{1}{6} \sqrt{6}\right)$ and $\gamma \in$ $(0,1)$. Then there exist $\varepsilon_{0}>0$ and $c_{1}, c_{2}>0$ such that for every $\varepsilon \in\left[0, \varepsilon_{0}\right]$ and $a \in[0, \bar{a}]$ the following holds.

If $\Omega$ is $\varepsilon$-close in $C^{2, \gamma}$-sense to $\Omega_{a}$, then the Green function $G_{\Omega}$ of (1.1) satisfies

$$
0<c_{1} D_{\Omega}(x, y) \leq G_{\Omega}(x, y) \leq c_{2} D_{\Omega}(x, y) \text { for every } x, y \in \Omega,
$$

where

$$
D_{\Omega}(x, y)=d_{\Omega}(x) d_{\Omega}(y) \min \left\{1, \frac{d_{\Omega}(x) d_{\Omega}(y)}{|x-y|^{2}}\right\} .
$$


Remark 2.6 In [7] the same estimates from above of $G_{\Omega}$ are given but with more regularity required at the boundary. Thanks to the $\varepsilon$-closeness we get a better estimate from below and the same from above with less assumptions on the boundary.

The proof consists of several steps and uses similar arguments as in [12] for a disk. For convenience we summarize the main parts here.

We first show that $\varepsilon$-closeness in $C^{2, \gamma}$-sense of $\Omega$ to $\Omega_{a}$ implies the existence of a biholomorphic map $\varphi_{a}: \Omega_{a} \rightarrow \Omega$ such that

$$
\left\|\varphi_{a}-I d\right\|_{C^{2, \gamma^{\prime}}\left(\bar{\Omega}_{a}\right)} \leq \delta(\varepsilon) \text { for } 0<\gamma^{\prime}<\gamma .
$$

Next, through this conformal mapping $\varphi_{a}$ problem (1.1) on $\Omega$ is transformed into the following problem on $\Omega_{a}$ :

$$
\left\{\begin{aligned}
\left(\Delta^{2}+A\right) u & =\tilde{f} \text { in } \Omega_{a}, \\
u & =0 \text { on } \partial \Omega_{a}, \\
\frac{\partial}{\partial \nu} u & =0 \text { on } \partial \Omega_{a}
\end{aligned}\right.
$$

where $A$ is a lower order perturbation of the biharmonic operator. See [13, Remark after Theorem 5.1]. From (2.7) one also has that there exists a $\delta_{1}=\delta_{1}(\varepsilon)>0$ such that the coefficients of $A$ in (2.8) satisfy

$$
\sup _{|\alpha| \leq 3}\left\|A_{\alpha}\right\|_{\infty} \leq \delta_{1}
$$

We then see that the positivity of the Green function associated to problem (2.8) implies the positivity of the Green function associated to problem (1.1) thanks to the properties of conformal maps ([21]). Hence, instead of proving directly Theorem 2.5 we prove the following result.

\section{Theorem 2.7 (Perturbation of $\Delta^{2}$ by lower order terms) Suppose}

that $a \in[0, \bar{a}]$ with $\bar{a}$ as in Theorem 2.5 and consider problem (2.8) with $A=\sum_{|\alpha| \leq 3} A_{\alpha} D^{\alpha}, A_{\alpha} \in C\left(\bar{\Omega}_{a}\right)$ and let $G_{\Omega_{a}, A}$ the Green function associated to (2.8).

Then there exists $\eta_{0}>0$ such that, whenever $\left\|A_{\alpha}\right\|_{\infty} \leq \eta_{0}$ for all $\alpha$ with $|\alpha| \leq 3$, the Green function associated to (2.8) is positive. Moreover, there exist $d_{1}, d_{2}>0$ such that, with $D_{\Omega_{a}}(x, y)$ as in (2.6), the following holds:

$$
d_{1} D_{\Omega_{a}}(x, y) \leq G_{\Omega_{a}, A}(x, y) \leq d_{2} D_{\Omega_{a}}(x, y)
$$


Theorem 2.7 says that if the lower order perturbation of the biharmonic operator is small then the positivity preserving property of system (2.8) in $\Omega_{a}$ follows from the positivity preserving property of problem (1.1) on the same domain.

A result similar to Theorem 2.7 was proven in [13] for the polyharmonic Dirichlet boundary value problem on the unit disk $B$. The main ingredient of the proof are appropriate estimates of

$$
H_{B}^{k}(x, y, z):=\frac{G_{B}(x, z)\left|D_{z}^{k} G_{B}(z, y)\right|}{G_{B}(x, y)},
$$

which were proved in [12]. Notice that in [12] one considers $\Omega$ being a ball. The only place however where that fact is used is in the explicit estimates of $H_{B}^{k}$. Indeed all the other arguments can be applied to any planar smooth domain $\Omega$ whose Green function is positive in the strict sense as in the left hand side of (2.9). Hence to prove Theorem 2.7 we first show that $H_{\Omega_{a}}^{k}$ (that is the quotient in (2.10) calculated for $G_{\Omega_{a}}$ ) satisfies the same estimates as $H_{B}^{k}$ and then refer to the work in [13].

In the next section we construct the conformal mapping from " $\Omega \varepsilon$ close to $\Omega_{a}$ " to the limaçon $\Omega_{a}$ and we state the equivalence of Theorem 2.5 and Theorem 2.7. Then we prove the perturbation result of Theorem 2.7 .

\subsubsection{Conformal transformation}

In this subsection we prove that problem (1.1) on $\Omega$ that is $\varepsilon$-close to $\Omega_{a}$, corresponds to a problem of the type (2.8) on $\Omega_{a}$ with the coefficients of $A$, the lower order perturbation of $\Delta^{2}$, being small. Or, to be more precise, there is a function $\varepsilon \mapsto \delta(\varepsilon)$ with $\delta(\varepsilon) \rightarrow 0$ when $\varepsilon \downarrow 0$, such that

$$
\Omega \varepsilon \text {-close in } C^{2, \gamma} \text {-sense to } \Omega_{a} \Longrightarrow \sup _{|\alpha| \leq 3}\left\|A_{\alpha}\right\|_{\infty} \leq \delta(\varepsilon) \text {. }
$$

Or in other words, that Theorem 2.7 implies Theorem 2.5.

The first step consist of proving existence of a biholomorphic map from the limaçon to a domain $\varepsilon$-close to the limaçon which is near the identity in $C^{2, \gamma}$-sense. 
Proposition 2.8 For all $\delta>0, \gamma \in(0,1)$ and $\bar{a} \in\left[0, \frac{1}{6} \sqrt{6}\right)$ there exists $\bar{\varepsilon}:=\bar{\varepsilon}(\delta, \bar{a}, \gamma)>0$ such that for all $\varepsilon \in[0, \bar{\varepsilon}]$ and $a \in[0, \bar{a}]$ we have the following.

If $\Omega$ is $\varepsilon$-close in $C^{2, \gamma}$-sense to $\Omega_{a}$ then there is a biholomorphic map $\varphi_{a}: \bar{\Omega}_{a} \rightarrow \bar{\Omega}$, with $\varphi_{a} \in C^{2, \gamma}\left(\bar{\Omega}_{a}\right)$ and $\varphi_{a}^{-1} \in C^{2, \gamma}(\bar{\Omega})$, such that

$$
\left\|\varphi_{a}-I d\right\|_{C^{2, \gamma}\left(\bar{\Omega}_{a}\right)} \leq \delta \text {. }
$$

The proof of Proposition 2.8 consist of the following three lemmas.

Since $a \leq \bar{a}<\frac{1}{6} \sqrt{6}<\frac{1}{2}$ one may check that the map $h_{a}$, defined in (2.1), is conformal and one-to-one on the domain

$$
B_{\sqrt{1.5}}:=\left\{x \in \mathbb{R}^{2}:\|x\|_{2}<\sqrt{1.5}\right\} .
$$

We choose the value $\varepsilon_{1} \in(0,1)$ such that, if $\Omega$ is $\varepsilon$-close in $C^{2, \gamma}$-sense to $\Omega_{a}$ for $\varepsilon \in\left(0, \varepsilon_{1}\right)$, then $h_{a}^{-1}(\Omega) \subset B_{\sqrt{1.5}}$. It follows that $h_{a}^{-1}$ is a conformal map on any domain $\Omega$ which is $\varepsilon$-close in $C^{2, \gamma}$-sense to the limaçon for $\varepsilon<\varepsilon_{1}$.

Lemma 2.9 There exists $\varepsilon_{1}>0$ and $c_{1}>0$ such that for $\varepsilon \in\left[0, \varepsilon_{1}\right]$ the following holds. Let $a \in[0, \bar{a}]$ and $\gamma \in(0,1)$. If $\Omega$ is $\varepsilon$-close in $C^{2, \gamma}$-sense to $\Omega_{a}$, then $\Omega^{h_{a}}:=h_{a}^{-1}(\Omega)$ is $c_{1} \varepsilon$-close in $C^{2, \gamma}$-sense to the disk $B$.

Proof: Let $h_{a}$ be the conformal map defined in (2.1). One directly checks that for $a(1+\nu) \in\left(0, \frac{1}{2}\right)$ the mapping $h_{a}: B_{1+\nu}(0) \rightarrow h_{a}\left(B_{1+\nu}(0)\right)$ is a $C^{\infty}$-diffeomorphism. So for $a \in[0, \bar{a}]$ the norms $\left\|h_{a}\right\|_{C^{4}(\bar{B})}$ and $\left\|h_{a}^{-1}\right\|_{C^{4}\left(\Omega_{a}\right)}$ are uniformly bounded. Let $g$ be a $C^{2, \gamma}$-mapping, $g: \bar{\Omega}_{a} \rightarrow$ $\bar{\Omega}$, such that $\|g-I d\|_{C^{2, \gamma}\left(\bar{\Omega}_{a}\right)} \leq \varepsilon$. We define the map $f: \bar{B} \rightarrow \bar{\Omega}^{h_{a}}$ by $f(x):=\left(h_{a}^{-1} \circ g \circ h_{a}\right)(x)$ (see Figure 2.3). Then there exists a positive constant $c_{1}$ such that $\|f-I d\|_{C^{2, \gamma}(\bar{B})} \leq c_{1} \varepsilon$.

In the following $\Omega^{h_{a}}$ denotes $h_{a}^{-1}(\Omega)$.

Lemma 2.10 Let $\gamma \in(0,1)$. Then for every $\delta>0$ there exist $\varepsilon_{2}:=$ $\varepsilon_{2}(\delta, \gamma, \bar{a})>0$ such that for every $\varepsilon \in\left(0, \varepsilon_{2}\right)$ and $a \in[0, \bar{a}]$ the following holds. If $\Omega$ is $\varepsilon$-close in $C^{2, \gamma}$-sense to $\Omega_{a}$, then there exists a biholomorphic mapping $\varphi: \bar{B} \rightarrow \bar{\Omega}^{h_{a}}$ with $\varphi \in C^{2, \gamma}(\bar{B}), \varphi^{-1} \in C^{2, \gamma}\left(\bar{\Omega}^{h_{a}}\right)$ and such that

$$
\|\varphi-I d\|_{C^{2, \gamma}(\bar{B})} \leq \delta \text {. }
$$




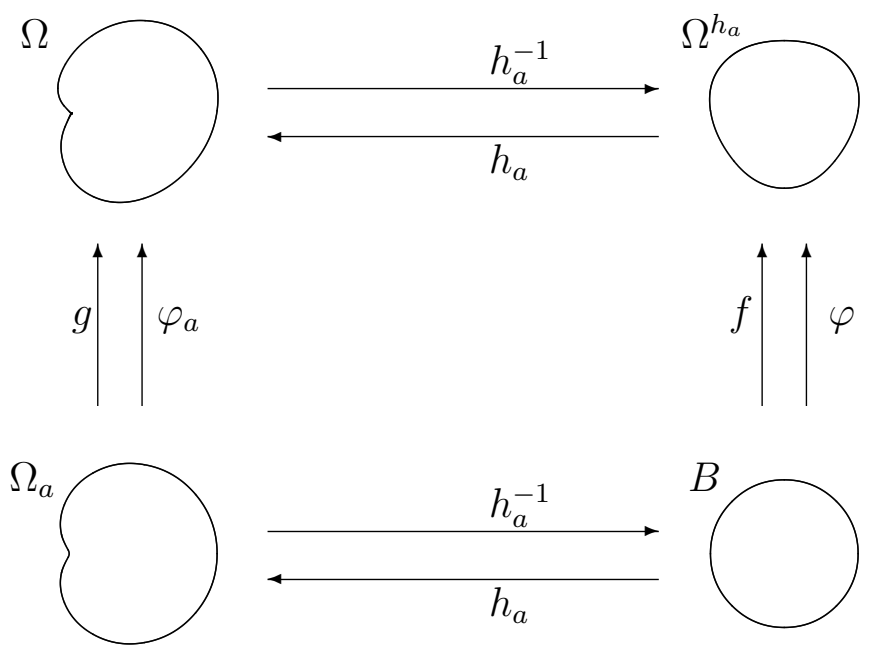

Figure 2.3 The mappings between $\Omega, \Omega_{a}, B$ and $\Omega^{h_{a}}$.

Proof: From Lemma 2.9 it follows that $\Omega^{h_{a}}$ is $c_{1} \varepsilon$-close to $B$. Applying Proposition A.1 we have that there exists $\varepsilon_{0}>0$ such that " $\Omega^{h_{a}} c_{1} \varepsilon$-close to $B$ " for $c_{1} \varepsilon \in\left(0, \varepsilon_{0}\right)$ implies the existence of a biholomorphic mapping $\varphi: \bar{B} \rightarrow \bar{\Omega}^{h_{a}}$ with $\varphi \in C^{2, \gamma}(\bar{B}), \varphi^{-1} \in C^{2, \gamma}\left(\bar{\Omega}^{h_{a}}\right)$ and such that

$$
\|\varphi-I d\|_{C^{2, \gamma}(\bar{B})} \leq \delta \text {. }
$$

The claim follows by taking $\varepsilon_{2}=\min \left\{\varepsilon_{1}, c_{1}^{-1} \varepsilon_{0}\right\}$.

Lemma 2.11 Let $\gamma \in(0,1)$. Then for every $\delta>0$ there exist $\varepsilon_{3}:=$ $\varepsilon_{3}(\delta, \gamma, \bar{a})>0$ such that for every $\varepsilon \in\left(0, \varepsilon_{3}\right)$ and $a \in[0, \bar{a}]$ the following holds.

If $\Omega$ is $\varepsilon$-close in $C^{2, \gamma}$-sense to $\Omega_{a}$, then there exists a biholomorphic mapping $\varphi_{a}: \bar{\Omega}_{a} \rightarrow \bar{\Omega}$ with $\varphi_{a} \in C^{2, \gamma}\left(\bar{\Omega}_{a}\right), \varphi_{a}^{-1} \in C^{2, \gamma}(\bar{\Omega})$ such that

$$
\left\|\varphi_{a}-I d\right\|_{C^{2, \gamma}\left(\bar{\Omega}_{a}\right)} \leq \delta .
$$

Proof: We denote $\varphi_{a}$ the map from $\bar{\Omega}_{a}$ to $\bar{\Omega}$ given by

$$
\varphi_{a}(x):=\left(h_{a} \circ \varphi \circ h_{a}^{-1}\right)(x) .
$$


Here $\varphi$ is the conformal map of Lemma 2.10. The map $\varphi_{a}$ is biholomorphic as a composition of biholomorphic maps. Furthermore we have $\varphi_{a} \in$ $C^{2, \gamma}\left(\bar{\Omega}_{a}\right)$ and $\varphi_{a}^{-1} \in C^{2, \gamma}(\bar{\Omega})$ since $\varphi \in C^{2, \gamma}(\bar{B})$ and $\varphi^{-1} \in C^{2, \gamma}\left(\bar{\Omega}^{h_{a}}\right)$.

By the way the holomorphic map $\varphi_{a}$ is defined one finds that there exists a positive constant $K$ such that

$$
\left\|\varphi_{a}-I d\right\|_{C^{2, \gamma}\left(\bar{\Omega}_{a}\right)} \leq K\|\varphi-I d\|_{C^{2, \gamma}(\bar{B})}
$$

The claim follows by choosing $\varepsilon_{3}=\varepsilon_{2}(\delta / K, \gamma, \bar{a})$ with $\varepsilon_{2}$ as defined in Lemma 2.10.

Remark 2.12 Notice that Proposition 2.8 follows from Lemma 2.11.

We are now ready to prove that the positivity preserving property of problem (2.8) with a small perturbation of $\Delta^{2}$ on $\Omega_{a}$ implies the positivity preserving property of problem (1.1) on $\Omega \varepsilon$-close in $C^{2, \gamma}$-sense to $\Omega_{a}$.

Corollary 2.13 Let $\gamma \in(0,1)$. For every $\delta>0$ small enough and $a \in$ $[0, \bar{a}]$ there exists $\varepsilon_{0}>0$ such that for $\varepsilon \in\left[0, \varepsilon_{0}\right)$ the following holds.

If $\Omega$ is $\varepsilon$-close in $C^{2, \gamma}$-sense to the limaçon $\Omega_{a}$ and the coefficients of the operator A satisfy

$$
\sup _{|\alpha| \leq 3}\left\|A_{\alpha}\right\|_{\infty} \leq \delta
$$

then the positivity of the Green function associated to problem (2.8) on $\Omega_{a}$ implies the positivity of the Green function associated to problem (1.1) on $\Omega$.

Proof: To prove the claim we show that problem (1.1) on $\Omega \varepsilon$-close in $C^{2, \gamma}$-sense to $\Omega_{a}$ can be "transformed" into problem (2.8) on $\Omega_{a}$ with the coefficients of the lower order operator $A$ satisfying (2.11).

Let $u$ be solution of problem (1.1) on $\Omega$. Consider $\delta_{0}<\min \left\{\frac{1}{2}, 2^{-7} \delta\right\}$. By Proposition 2.8 we know that there exists a $\varepsilon_{0}=\varepsilon_{0}\left(\delta_{0}\right)>0$ such that for $\varepsilon \in\left[0, \varepsilon_{0}\right)$ we have the following. If $\Omega$ is $\varepsilon$-close to $\Omega_{a}$ in $C^{2, \gamma}$-sense then there exists a conformal map $\varphi_{a}: \bar{\Omega}_{a} \rightarrow \bar{\Omega}$ such that

$$
\left\|\varphi_{a}-I d\right\|_{C^{2, \gamma}\left(\bar{\Omega}_{a}\right)} \leq \delta_{0}
$$


We define the function $v_{a}(x):=u \circ \varphi_{a}(x)$ on $\Omega_{a}$. Clearly $u>0$ if and only if $v_{a}>0$. Since $\varphi_{a}$ is a conformal map, the function $v_{a}$ satisfies

$$
\left\{\begin{aligned}
\Delta^{2} v_{a}-2 \nabla\left|\varphi_{a}^{\prime}\right|^{2} \cdot \nabla \frac{\Delta v_{a}}{\left|\varphi_{a}^{\prime}\right|^{2}}-4\left|\varphi_{a}^{\prime \prime}\right|^{2} \frac{\Delta v_{a}}{\left|\varphi_{a}^{\prime}\right|^{2}} & =\left|\varphi_{a}^{\prime}\right|^{4} f \circ \varphi_{a} & & \text { in } \Omega_{a}, \\
v_{a} & =0 & & \text { on } \partial \Omega_{a}, \\
\frac{\partial}{\partial \nu} v_{a} & =0 & & \text { on } \partial \Omega_{a},
\end{aligned}\right.
$$

where $\varphi_{a}^{\prime}$ denotes the complex derivative of $\varphi_{a}$. Hence $v_{a}$ is solution of a problem as in (2.8). The coefficients of the lower order perturbation of $\Delta^{2}$ in (2.12) satisfy (2.11) by the choice of $\delta_{0}$.

Remark 2.14 Notice that since we are working with conformal mappings it is sufficient to have $C^{2, \gamma}$-closeness in order to transform problem (1.1) into problem (2.8). Working with general transformations fourth order derivatives would appear and $C^{4, \gamma}$-closeness would be necessary.

As a consequence of Corollary 2.13, Theorem 2.5 will follow from Theorem 2.7.

\subsubsection{Proof of the perturbation theorem}

In [13] Theorem 2.7 has been proven in the unit disk (that is $\Omega_{0}$ ). We now give a sketch of the proof for $\Omega_{a}, a \in[0, \bar{a}]$ by following similar steps.

First we state some estimates for (2.10) with $G_{B}$ replaced by $G_{\Omega_{a}}$.

Theorem 2.15 Let $k=\left(k_{1}, k_{2}\right)$ with $k_{1}, k_{2} \in \mathbb{N}$ and $|k| \leq 3$. The following estimates hold for any $a \in[0, \bar{a}]$ and $x, y, z \in \Omega_{a}$.

$$
\begin{array}{ll}
\text { 1. If }|k|=3 \text {, then } & \frac{G_{\Omega_{a}}(x, z)\left|D_{z}^{k} G_{\Omega_{a}}(z, y)\right|}{G_{\Omega_{a}}(x, y)} \preceq \frac{1}{|x-z|}+\frac{1}{|y-z|} \text {. } \\
\text { 2. If }|k|=2 \text {, then } \quad \frac{G_{\Omega_{a}}(x, z)\left|D_{z}^{k} G_{\Omega_{a}}(z, y)\right|}{G_{\Omega_{a}}(x, y)} \preceq \log \left(\frac{3}{|z-y|}\right) . \\
\text { 3. If }|k| \leq 1 \text {, then } \quad \frac{G_{\Omega_{a}}(x, z)\left|D_{z}^{k} G_{\Omega_{a}}(z, y)\right|}{G_{\Omega_{a}}(x, y)} \preceq 1 .
\end{array}
$$

Proof: With the same method as has been used in [13] the result follows from the optimal estimate from below for $G_{\Omega_{a}}$, which has been proved in 
[7] (see Proposition B.1), and from the estimates of the derivatives of the Green function, which have been proved in [8] (see Proposition B.2).

Let $\mathcal{G}_{\Omega}$ denote the Green operator associated to problem (1.1) in $\Omega$, that is

$$
\mathcal{G}_{\Omega} f(x):=\int_{\Omega} G_{\Omega}(x, y) f(y) d y .
$$

By the estimate in Theorem 2.15 one may observe that the derivatives of the Green function have an integrable singularity. Hence one finds the following two corollaries of Theorem 2.15.

Corollary 2.16 There exists $M \in \mathbb{R}^{+}$such that for any $0 \leq f \in L^{p}\left(\Omega_{a}\right)$ with $p \geq 1$ and $k=\left(k_{1}, k_{2}\right) \in \mathbb{N}^{2}$ with $0 \leq|k| \leq 3$, the following estimate holds for all $a \in[0, \bar{a}]$

$$
\left|\left(\mathcal{G}_{\Omega_{a}} D^{k} \mathcal{G}_{\Omega_{a}} f\right)(x)\right| \leq M\left(\mathcal{G}_{\Omega_{a}} f\right)(x) \text { for all } x \in \Omega_{a} .
$$

Corollary 2.17 Let $a \in[0, \bar{a}]$ and $\eta>0$ be such that the coefficients of $A$ in (2.8) satisfy $\left\|A_{\alpha}\right\|_{\infty} \leq \eta$ for all $|\alpha| \leq 3$. Then for any $0 \leq f \in L^{p}\left(\Omega_{a}\right)$ with $p \geq 1$

$$
\left|\left(\mathcal{G}_{\Omega_{a}} A \mathcal{G}_{\Omega_{a}} f\right)(x)\right| \leq 10 M \eta\left(\mathcal{G}_{\Omega_{a}} f\right)(x) \text { for all } x \in \Omega_{a},
$$

and furthermore

$$
\left|\left(\left(\mathcal{G}_{\Omega_{a}} A\right)^{i} \mathcal{G}_{\Omega_{a}} f\right)(x)\right| \leq(10 M \eta)^{i}\left(\mathcal{G}_{\Omega_{a}} f\right)(x) \text { for all } x \in \Omega_{a},
$$

where $M$ is the constant of Corollary 2.16.

For the proofs we refer to [13, Cor.4.2, Lem.5.4-5.5].

Proof of Proof of Theorem 2.7: Let $u$ be a solution of (2.8). Proceeding as in [13, Lemma 5.3] one finds that there exists a $\eta_{1}>0$ such that $\left(\mathcal{I}+\mathcal{G}_{\Omega_{a}} A\right)^{-1}$ is well defined when the coefficients of $A$ satisfy $\left\|A_{\alpha}\right\|_{\infty} \leq$ $\eta_{1}$ for $|\alpha| \leq 3$. We have $u=-\mathcal{G}_{\Omega_{a}} A u+\mathcal{G}_{\Omega_{a}} f$ or $u=\left(\mathcal{I}+\mathcal{G}_{\Omega_{a}} A\right)^{-1} \mathcal{G}_{\Omega_{a}} f$ and may formally write

$$
\begin{aligned}
& \mathcal{G}_{\Omega_{a}, A}=\left(\mathcal{I}+\mathcal{G}_{\Omega_{a}} A\right)^{-1} \mathcal{G}_{\Omega_{a}} \\
= & \mathcal{G}_{\Omega_{a}}-\mathcal{G}_{\Omega_{a}} A \mathcal{G}_{\Omega_{a}}+\left(\mathcal{G}_{\Omega_{a}} A\right)^{2} \mathcal{G}_{\Omega_{a}}-\left(\mathcal{G}_{\Omega_{a}} A\right)^{3} \mathcal{G}_{\Omega_{a}}+\ldots
\end{aligned}
$$


Using Corollary 2.17 from (2.13) taking $\eta_{0}=\min \left\{\frac{1}{30 M}, \eta_{1}\right\}$ and $\eta \leq \eta_{0}$ the series converges and we get

$$
\frac{1}{2} \mathcal{G}_{\Omega_{a}} \leq \mathcal{G}_{\Omega_{a}, A} \leq \frac{3}{2} \mathcal{G}_{\Omega_{a}}
$$

The estimate in (2.9) follows directly from (2.14) and Proposition B.1.

Remark 2.18 For the problem

$$
\left\{\begin{aligned}
\left(\Delta^{2}+A\right) u & =f \text { in } \Omega \\
u & =0 \text { on } \partial \Omega \\
\frac{\partial}{\partial \nu} u & =0 \text { on } \partial \Omega
\end{aligned}\right.
$$

with $\Omega \varepsilon$-close in $C^{2, \gamma}$-sense to $\Omega_{a}$ for $a \in[0, \bar{a}]$ and with $A$ the lower order perturbation of the bilaplacian such that $\left\|A_{\alpha}\right\|_{\infty} \leq \eta$ for $|\alpha| \leq 3$, the result stated in Theorem 2.7 is still valid for $\varepsilon$ and $\eta$ sufficiently small.

\section{An approximate filling of the domain by per- turbed limaçons}

In this section we prove that a sufficiently smooth bounded two-dimensional domain can be approximated by limaçon-like domains in the sense we want. That is, we will construct a finite number of domains $E_{j}$ such that:

1. the union of $E_{j}$ covers $\Omega$ near $\partial \Omega$;

2. the union of $\partial E_{j}$ covers the boundary $\partial \Omega$;

3. each $E_{j}$ is close in $C^{2, \gamma}$-sense to a limaçon $\Omega_{a, R}$ with $a \in[0, \bar{a}]$ in a uniform way;

4. the $E_{j}$ uniformly satisfy the uniform $C^{4, \gamma}$ regularity condition in $a \in[0, \bar{a}]$.

Although this covering looks like it exactly fills up the domain this will not be guaranteed. Indeed, some parts of the covering may lie outside of $\Omega$. The precise statement is given in Theorem 3.14. 


\subsection{Local approximation}

We first show that for each $z_{0}$ on $\partial \Omega$ there exists a domain $\varepsilon$-close to a limaçon which boundary intersects $\partial \Omega$ in a neighborhood of $z_{0}$. In order to do that it will be convenient to use local systems of Cartesian coordinates. The following lemma lists some technical results.

Lemma 3.1 Let $\ell \geq 2$ and $\Omega$ be a domain in $\mathbb{R}^{2}$ satisfying the uniform $C^{\ell, \alpha}$ regularity condition, Definition 1.11 , with constant $M$ and mappings $\varphi_{j} \in C^{\ell, \alpha}, j \in J$. Let $\rho_{\Omega}$ be as in Notation 1.12 and set $x_{\rho_{\Omega}}:=\frac{\sqrt{3}}{2} \rho_{\Omega}$.

Then for every $z_{0} \in \partial \Omega$ there exists a local Cartesian coordinates system and a function $g_{z_{0}} \in C^{\ell, \alpha}, g_{z_{0}}:\left[-x_{\rho_{\Omega}}, x_{\rho_{\Omega}}\right] \rightarrow \mathbb{R}$, such that:

1. $z_{0}=(0,0)$;

2. the $x$-axis is tangential to $\partial \Omega$ in $z_{0}$;

3. the $y$-axis has the direction of the internal normal to $\partial \Omega$ in $z_{0}$;

4. $B_{\frac{1}{2} \rho_{\Omega}}\left(z_{0}\right) \cap \partial \Omega \subset\left\{(x, y): x \in\left[-x_{\rho_{\Omega}}, x_{\rho_{\Omega}}\right]\right.$ and $\left.y=g_{z_{0}}(x)\right\}$;

5. $\left\|g_{z_{0}}\right\|_{C^{\ell, \alpha}\left[-x_{\rho_{\Omega}, x_{\rho_{\Omega}}}\right]} \leq 2(\ell+1) M$.

Remark 3.2 Observe that the function $g_{z_{0}}$ satisfies $\left|g_{z_{0}}^{\prime}(x)\right| \leq \frac{1}{\sqrt{3}}$.

We skip the rather technical proof of Lemma 3.1.

In the following theorem we will state that for every point of the boundary of a domain satisfying the uniform $C^{4, \alpha}$ regularity condition there exists a limaçon $\Omega_{a, R}$ that approximates $\partial \Omega$ in the point in $C^{2}$-sense. Furthermore we will construct a domain $\tilde{\Omega}$ that is $\varepsilon$-close to the limaçon $\Omega_{a, R}$ and which boundary coincides with $\partial \Omega$ in a neighborhood of that point. By construction $\tilde{\Omega}$ is a domain satisfying the uniform $C^{4, \alpha}$ regularity condition with constant $M_{1}$ where $M_{1}$ depends only on $M$ and $\rho_{\Omega}$.

For the purpose of a uniform statement we will have to rescale to limaçons of 'unit' size. In order to do so we define for a given $f$ the scaled function:

$$
f^{R}(x, y):=R^{-1} f(R x, R y) \text { for } R \in \mathbb{R}^{+} .
$$

Theorem 3.3 Assume that the following holds for some $\alpha, \gamma \in(0,1)$ : 
i. $\Omega \subset \mathbb{R}^{2}$ is a simply connected domain satisfying the uniform $C^{4, \alpha}$ regularity condition with constant $M$;

ii. $g_{z_{0}} \in C^{4, \alpha}$ for $z_{0} \in \partial \Omega$ are functions that describe the boundary of $\Omega$ as in Lemma 3.1 and set $R:=\min \left\{\frac{1}{2}\left(\max _{z_{0} \in \partial \Omega}\left\|g_{z_{0}}^{\prime \prime}\right\|_{\infty}\right)^{-1}, 1\right\}$;

iii. $\varepsilon>0$ is such that for all $\tilde{\Omega}$ which are $\varepsilon$-close to $\Omega_{a, 1}$ in $C^{2, \gamma}$ sense with $a \in\left[\frac{3}{16}, \frac{5}{16}\right]$, the Green function associated to problem (1.1) on $\tilde{\Omega}$ is positive.

Then there is $\delta=\delta\left(M, \rho_{\Omega}^{-1}, \varepsilon, \gamma\right) \in\left(0, \frac{1}{16} R\right)$ such that the following holds. For every $z_{0} \in \partial \Omega$ there exist $a \in\left[\frac{3}{16}, \frac{5}{16}\right]$, a limaçon $\Omega_{a, R}$ and $a$ $C^{4, \alpha}$ map $f_{a, R}: \bar{\Omega}_{a, R} \rightarrow f_{a, R}\left(\bar{\Omega}_{a, R}\right)$ such that:

1. $\partial \Omega \cap B_{\delta}\left(z_{0}\right)=\partial\left(f_{a, R}\left(\Omega_{a, R}\right)\right) \cap B_{\delta}\left(z_{0}\right)$;

2. the map $f_{a, R}^{R}:=\left(f_{a, R}\right)^{R}$ is $\varepsilon$-close in $C^{2, \gamma}$-sense to the identity on $\bar{\Omega}_{a, 1}:\left\|f_{a, R}^{R}-I d\right\|_{C^{2, \gamma}\left(\bar{\Omega}_{a, 1}\right)} \leq \varepsilon$

3. the map $f_{a, R}^{R}$ is $C^{4, \alpha}$ bounded by some $A=A\left(M, \rho_{\Omega}^{-1}, \varepsilon, \gamma\right)$ : that is, $\left\|f_{a, R}^{R}\right\|_{C^{4, \alpha}\left(\bar{\Omega}_{a, 1}\right)} \leq A$.

Remark 3.4 We construct a $C^{4, \alpha}$ mapping $f_{a, R}: \bar{\Omega}_{a, R} \rightarrow f_{a, R}\left(\bar{\Omega}_{a, R}\right)$ in order that $f_{a, R}\left(\bar{\Omega}_{a, R}\right)$ is a domain satisfying the uniform $C^{4, \alpha}$ regularity condition with constant $M_{1}$ where $M_{1}=M_{1}\left(M, \rho_{\Omega}^{-1}, \varepsilon, \gamma\right)$. Using the result in [9] it should be possible to relax the regularity of the boundary to $C^{4}$.

Remark 3.5 In order to approximate $\partial \Omega$ with limaçons in $C^{2, \gamma}$-sense it is sufficient that $\Omega$ satisfies the uniform $C^{2, \alpha}$ regularity condition for $\alpha>\gamma$.

Remark 3.6 The $R$ defined in Theorem 3.3 depends on $\Omega$ via the constant $M$ of the uniform $C^{4, \alpha}$ regularity condition.

Corollary 3.7 Assume that $\Omega, \alpha, \gamma, \varepsilon$ are such that the hypotheses of Theorem 3.3 hold true and let $R$ as defined in that theorem. Then there is $\delta>0$ such that for every $z_{0} \in \partial \Omega$ there exists a domain $E_{z_{0}}$ that satisfies the following: 
1. $E_{z_{0}}$ satisfies the uniform $C^{4, \alpha}$ regularity condition with constant $M_{1}=M_{1}\left(M, \rho_{\Omega}^{-1}, \varepsilon, \gamma\right)>0$;

2. $E_{z_{0}}$ is $\varepsilon$-close in $C^{2, \gamma}$-sense to a limaçon $\Omega_{a, R}$ with $a \in\left[\frac{3}{16}, \frac{5}{16}\right]$;

3. $z_{0} \in\left(\bar{E}_{z_{0}} \cap \partial \Omega\right)^{\circ, \partial \Omega}$.

Furthermore, letting $K_{z_{0}}$ be the component of $\left(\bar{E}_{z_{0}} \cap \partial \Omega\right)^{\circ, \partial \Omega}$ that contains $z_{0}$ :

4. $B_{\delta}\left(z_{0}\right) \cap \partial \Omega=B_{\delta}\left(z_{0}\right) \cap K_{z_{0}}$;

5. $E_{z_{0}}$ and $\Omega$ have the same outward normal for any $x \in K_{z_{0}}$.

The proof of Theorem 3.3 is divided into several steps. We first present the setting for a fixed $z_{0} \in \partial \Omega$. Let us consider the local system of coordinates near $z_{0}$ and the function $g_{z_{0}} \in C^{4, \alpha}$ given by Lemma 3.1 (in this case $l=4$ ). We will write $g_{z_{0}}=g$.

Let $\delta$ be a positive number such that

$$
\delta<\min \left\{1, \frac{x_{\rho_{\Omega}}}{4}, \frac{R}{16}\left(1-\frac{5}{16} \sqrt{3}\right)\right\} \text { and } \delta^{1-\gamma}<\varepsilon\left(C_{10} R^{1+\gamma}\right)^{-1} .
$$

Here $C_{10}$ is a positive constant that depends on $M$. We remark that $\delta$ depends on $\Omega$ through $\rho_{\Omega}^{-1}$ and $M$.
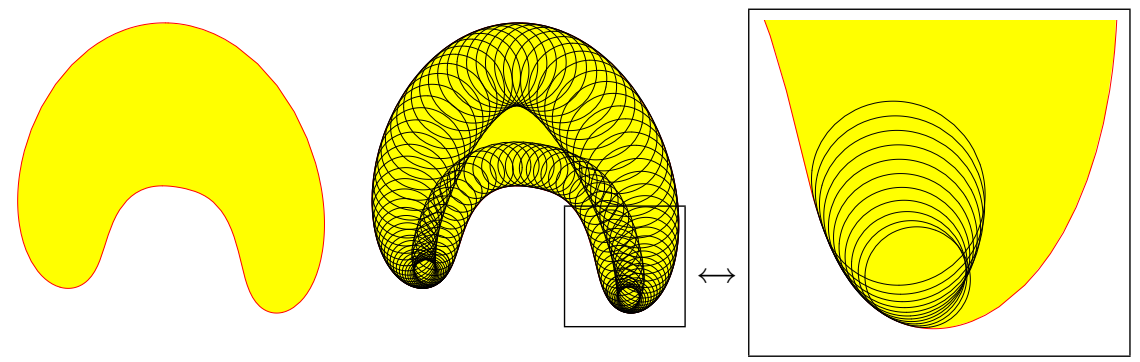

Figure 3.1 A domain, the finite number of approximated limaçons with their boundaries covering the boundary of the domain, and a zoomed view. 


\subsection{Approximation by a limaçon in one point}

There exists $a \in\left[\frac{3}{16}, \frac{5}{16}\right]$ such that $z_{0}=(0,0) \in \bar{\Omega}_{a, R}$ and

$$
k_{a, R}^{\prime \prime}(0)=g^{\prime \prime}(0) \text {, }
$$

where $k_{a, R} \in C^{\infty}$ is the map that describes, as in (2.3), the lower part of the limaçon.

In order to get that $\partial \Omega_{a, R}$ approximates the boundary of $\Omega$ in $(0,0)$ up to the second derivative, we have to impose the condition $g^{\prime \prime}(0)=k_{a, R}^{\prime \prime}(0)$. Using (2.4) this reads as

$$
g^{\prime \prime}(0)=\frac{1}{R} \frac{1-4 a}{(1-2 a)^{2}} .
$$

Since the map $a \mapsto \frac{1-4 a}{(1-2 a)^{2}}$ sends the interval $\left[\frac{3}{16}, \frac{5}{16}\right]$ onto $\left[-\frac{1}{2}, \frac{1}{2}\right]$ and it holds $\left|g^{\prime \prime}(0)\right| R \leq \frac{1}{2}$ by the definition of $R$, one finds that $a \in\left[\frac{3}{16}, \frac{5}{16}\right]$ exists such that (3.3) holds.

Note that $R$ is fixed and that it is sufficient to play with the parameter $a$ to fit the limaçon $\Omega_{a, R}$ to the domain $\Omega$ around $z_{0}$.

\subsection{Construction of the mapping $f_{a, R}$}

Again we fix some preliminaries. Let $x_{a}$ be the number defined in (2.2) and let us set $x_{a}^{*}:=\frac{1}{2}(1-\sqrt{3} a) \in\left(\frac{1}{5} x_{a}, \frac{1}{2} x_{a}\right)$. We introduce two cut-off functions:

1. $\varphi_{a, R} \in C^{\infty}(\mathbb{R})$ such that

$$
\begin{array}{cl}
\varphi_{a, R} \equiv 1 & \text { for }|x| \leq \frac{1}{2} x_{a}^{*} R \\
\varphi_{a, R} \equiv 0 & \text { for }|x| \geq x_{a}^{*} R \\
\left\|\varphi_{a, R}\right\|_{C^{k, \nu}} \leq \frac{D_{k, \nu}}{R^{k+\nu}} & \text { for } k=0, \ldots, 4 \text { and } \nu \in(0,1),
\end{array}
$$

with $D_{k, \nu}$ some positive constants;

2. $\psi_{a, \delta} \in C^{\infty}(\mathbb{R})$ such that

$$
\begin{array}{cl}
\psi_{a, \delta} \equiv 1 & \text { for }|x| \leq \delta \\
\psi_{a, \delta} \equiv 0 & \text { for }|x| \geq 2 \delta \\
\left\|\psi_{a, \delta}\right\|_{C^{k, \nu}} \leq \frac{D_{k, \nu}^{\prime}}{\delta^{k+\nu}} & \text { for } k=0, \ldots, 4 \text { and } \nu \in(0,1),
\end{array}
$$

with $D_{k, \nu}^{\prime}$ some positive constants. 
We define a $C^{4, \alpha}$-mapping $g_{\delta}$ on $\left[-R x_{a}, R x_{a}\right]$ that follows the boundary of $\Omega$ when $|x| \leq \delta$ and the boundary of the limaçon when $R x_{a}^{*} \leq$ $|x| \leq R x_{a}$ as follows:

$$
g_{\delta}(x):= \begin{cases}g(x) & \text { for } 0 \leq x \leq \delta, \\ k_{a, R}(x)+\left.\sum_{i=0}^{2} \frac{1}{i !}\left(g-k_{a, R}\right)^{(i)}\right|_{\delta}(x-\delta)^{i}+ & \\ +\left.\psi_{a, \delta}(x) \sum_{i=3}^{4} \frac{1}{i !}\left(g-k_{a, R}\right)^{(i)}\right|_{\delta}(x-\delta)^{i} & \text { for } \delta<x \leq 2 \delta, \\ k_{a, R}(x)+\left.\sum_{i=0}^{2} \frac{1}{i !}\left(g-k_{a, R}\right)^{(i)}\right|_{\delta}(x-\delta)^{i} & \text { for } 2 \delta<x \leq \frac{1}{2} R x_{a}^{*}, \\ k_{a, R}(x)+\left.\varphi_{a, R}(x) \sum_{i=0}^{2} \frac{1}{i !}\left(g-k_{a, R}\right)^{(i)}\right|_{\delta}(x-\delta)^{i} & \text { for } \frac{1}{2} R x_{a}^{*}<x \leq R x_{a}^{*}, \\ k_{a, R}(x) & \text { for } R x_{a}^{*}<x \leq R x_{a},\end{cases}
$$

and similarly for $x \in\left[-R x_{a}, 0\right]$.

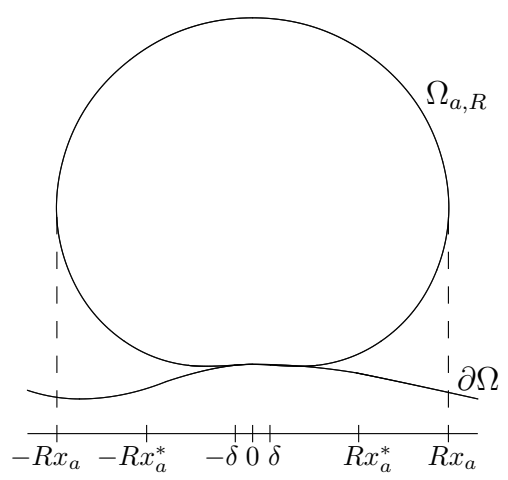

Figure 3.2 The limaçon that approximates in $(0,0)$ the behavior of $\partial \Omega$ up to the second derivative.

Remark 3.8 In the definition of $g_{\delta}$ we use two cut-off functions. The reason for this construction is that we want $g_{\delta}$ to be close to $k_{a, R}$ in $C^{2, \gamma}$ sense and also to be a $C^{4, \alpha}$-mapping. Indeed, one sees when considering 


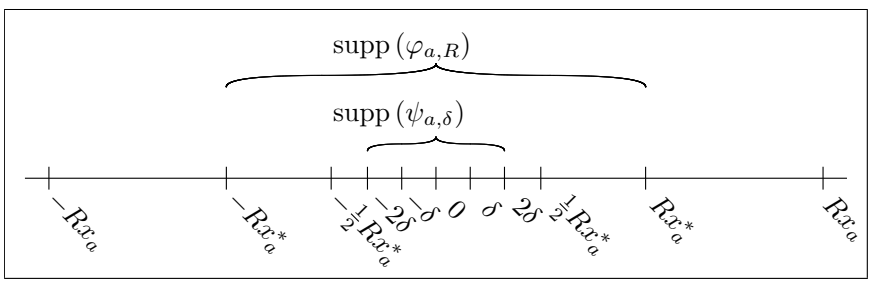

Figure 3.3 Scheme for the support of the cut-off functions $\varphi_{a, R}$ and $\psi_{a, \delta}$.

$\left\|g_{\delta}-k_{a, R}\right\|_{C^{2, \gamma}\left(-R x_{a}, R x_{a}\right)}$ that the terms $\left.\left(g-k_{a, R}\right)^{(i)}\right|_{\delta}$ have a different behavior for $i=0,1,2$ respectively for $i=3,4$. One cut-off function can be chosen independent of $\delta$ since we will show that for $i=0,1,2$ the term $\left.\left(g-k_{a, R}\right)^{(i)}\right|_{\delta}=O(\delta)$. While for $i=3,\left.4\left(g-k_{a, R}\right)^{(i)}\right|_{\delta}$ will be just bounded, and hence one needs a cut-off function depending on $\delta$ in order that the $C^{2, \gamma}$-norm of $g-k_{a, R}$ is an $O(\delta)$. By the way, close in $C^{2, \gamma}$-sense is needed for positivity; $C^{4, \alpha}$ is used in the regularity results.

We define the function $f_{a, R}: \bar{\Omega}_{a, R} \rightarrow f_{a, R}\left(\bar{\Omega}_{a, R}\right)$ by

$$
f_{a, R}(x, y)=\left(x, \frac{3 R-g_{\delta}(x)}{3 R-k_{a, R}(x)}(y-3 R)+3 R\right),
$$

which gives $\left(f_{a, R}-I\right)(x, y)=\left(0, \frac{k_{a, R}(x)-g_{\delta}(x)}{3 R-k_{a, R}(x)}(y-3 R)\right)$. By construction $f_{a, R} \in C^{4, \alpha}\left(\bar{\Omega}_{a, R}\right)$ and the boundary of $f_{a, R}\left(\Omega_{a, R}\right)$ coincides with $\partial \Omega$ in a neighborhood of $z_{0}=(0,0)$ of length at least $2 \delta$.

In the next paragraph we show that $f_{a, R}\left(\Omega_{a, R}\right)$ is $\varepsilon$-close to $\Omega_{a, R}$ in $C^{2, \gamma}$-sense and that $f_{a, R}\left(\Omega_{a, R}\right)$ satisfies the uniform $C^{4, \alpha}$ regularity condition.

Remark 3.9 Notice that $f_{a, R} \equiv I d$ for $(x, y) \in \bar{\Omega}_{a, R}$ with $|x| \geq R x_{a}^{*}$. While for $|x|<R x_{a}^{*}$ it holds that $f_{a, R} \equiv I d$ for $x=0$ only. The map $f_{a, R}$ also changes the boundary of $\Omega_{a, R}$ in a neighborhood of the point $(0,2 R)$. That is not a problem since one may notice from the expression of $f_{a, R}-I d$ that in the approximation only the term $\frac{k_{a, R}(x)-g_{\delta}(x)}{3 R-k_{a, R}(x)}$ plays a role. 


\subsection{The mapping is close to the identity in $C^{2, \gamma}$-sense}

In this section we will prove that $f_{a, R}^{R}$, which is the $f_{a, R}$ from (3.5) rescaled as in (3.1), satisfies

$$
\left\|f_{a, R}^{R}-I d\right\|_{C^{2, \gamma}\left(\bar{\Omega}_{a, 1}\right)} \leq \varepsilon
$$

By the results of the previous section and our choice of $\varepsilon$, it then follows that the Green function associated to problem (1.1) on $f_{a, R}\left(\Omega_{a, R}\right)$ is positive.

We first fix some notation. In the following $N_{1}$ and $N_{2}$ denote respectively

$$
\begin{aligned}
& N_{1}:=\left\|\frac{\partial^{4}}{\partial x^{4}} k_{a, R}-\frac{\partial^{4}}{\partial x^{4}} g\right\|_{C^{0}[-\delta, \delta]}+\left|\frac{\partial^{3}}{\partial x^{3}} k_{a, R}(0)-\frac{\partial^{3}}{\partial x^{3}} g(0)\right| \\
& N_{2}:=\left[\frac{\partial^{4}}{\partial x^{4}} k_{a, R}-\frac{\partial^{4}}{\partial x^{4}} g\right]_{C^{\alpha}[-\delta, \delta]}+\left|\frac{\partial^{4}}{\partial x^{4}} k_{a, R}(0)-\frac{\partial^{4}}{\partial x^{4}} g(0)\right|
\end{aligned}
$$

Notice that $N_{i}=N_{i}(M)$ for $i=1,2$. Indeed $R$ depends on $M$ and the dependence of $k_{a, R}$ on $a$ is continuous in $\left[\frac{3}{16}, \frac{5}{16}\right]$ and hence uniform.

We have

$$
\begin{gathered}
\left\|\frac{\partial^{i}}{\partial x^{i}} k_{a, R}-\frac{\partial^{i}}{\partial x^{i}} g\right\|_{C^{0}[-\delta, \delta]} \leq N_{1} \delta^{3-i} \text { for } i=0, \ldots, 3 \\
\text { and }\left\|\frac{\partial^{4}}{\partial x^{4}} k_{a, R}-\frac{\partial^{4}}{\partial x^{4}} g\right\|_{C^{0}[-\delta, \delta]} \leq N_{2}
\end{gathered}
$$

In order to prove (3.6) one has first to consider the effect of the scaling.

Proposition 3.10 Let $\gamma \in(0,1)$. The function $f_{a, R}^{R}$ satisfies

$$
\begin{aligned}
& \left\|f_{a, R}^{R}-I d\right\|_{C^{2, \gamma}\left(\bar{\Omega}_{a, 1}\right)} \leq 5\left\|\frac{k_{a, R}-g_{\delta}}{3 R-k_{a, R}}\right\|_{C^{0}\left[-R x_{a}^{*}, R x_{a}^{*}\right]}+ \\
& +5 R\left\|\frac{\partial}{\partial x} \frac{k_{a, R}-g_{\delta}}{3 R-k_{a, R}}\right\|_{C^{0}\left[-R x_{a}^{*}, R x_{a}^{*}\right]}+9 R^{2}\left\|\frac{\partial^{2}}{\partial x^{2}} \frac{k_{a, R}-g_{\delta}}{3 R-k_{a, R}}\right\|_{C^{0}\left[-R x_{a}^{*}, R x_{a}^{*}\right]} \\
& +4 R^{2+\gamma}\left[\frac{\partial^{2}}{\partial x^{2}} \frac{k_{a, R}-g_{\delta}}{3 R-k_{a, R}}\right]_{C^{\gamma}\left[-R x_{a}^{*}, R x_{a}^{*}\right]} .
\end{aligned}
$$

We postpone the proof of Proposition 3.10 to Appendix C.1.1. 
Proposition 3.11 Let $\gamma \in(0,1)$. There is $C_{10}=C_{10}(M)>0$ such that

$$
\left\|f_{a, R}^{R}-I d\right\|_{C^{2, \gamma}\left(\bar{\Omega}_{a, 1}\right)} \leq C_{10} R^{1+\gamma} \delta^{1-\gamma} .
$$

The right-hand side in (3.11) is less then $\varepsilon$ due to our choice of $\delta$ in (3.2).

In order to prove Proposition 3.11 we estimate the terms in the right hand side of (3.10) separately. The details of the proof are given in Appendix C.1.2.

\subsection{Bounded third and fourth derivative of the mapping}

In this section we derive the estimate of $\left\|f_{a, R}^{R}\right\|_{C^{4, \alpha}\left(\bar{\Omega}_{a, 1}\right)}$. Again this $f_{a, R}^{R}$ is the function $f_{a, R}$ from (3.5) rescaled as in (3.1). The estimate will imply that $f_{a, R}\left(\Omega_{a, R}\right)$ satisfies the uniform $C^{4, \alpha}$ regularity condition.

The effect of the scaling is as follows:

Proposition 3.12 Let $\alpha \in(0,1)$. There is $C_{11}=C_{11}(M)>0$ such that:

$$
\begin{aligned}
& \left\|f_{a, R}^{R}\right\|_{C^{4, \alpha}\left(\bar{\Omega}_{a, 1}\right)} \leq x_{a}+9+5 C_{11} \delta R+ \\
& +5 R^{3}\left\|\frac{\partial^{3}}{\partial x^{3}} \frac{k_{a, R}-g_{\delta}}{3 R-k_{a, R}}\right\|_{C^{0}\left(-R x_{a}^{*}, R x_{a}^{*}\right)}+ \\
& +6 R^{4}\left\|\frac{\partial^{4}}{\partial x^{4}} \frac{k_{a, R}-g_{\delta}}{3 R-k_{a, R}}\right\|_{C^{0}\left(-R x_{a}^{*}, R x_{a}^{*}\right)}+ \\
& +R^{3+\alpha}\left[(x, y) \mapsto(y-3 R) \frac{\partial^{4}}{\partial x^{4}} \frac{k_{a, R}-g_{\delta}}{3 R-k_{a, R}}\right]_{C^{\alpha}\left(\bar{\Omega}_{a, R}\right)} .
\end{aligned}
$$

We postpone the proof of Proposition 3.12 to Appendix C.2.1.

The estimate we are looking for is then:

Proposition 3.13 Let $\alpha \in(0,1)$. There is $C_{19}=C_{19}(M)>0$ such that:

$$
\left\|f_{a, R}^{R}\right\|_{C^{4, \alpha}\left(\bar{\Omega}_{a, 1}\right)} \leq C_{19} \frac{R^{3+\alpha}}{\delta^{1+\alpha}} .
$$

In order to prove Proposition 3.13 it is sufficient to find appropriate estimates for the terms in the right hand side of (3.12). The details of the proof are in Appendix C.2.2. 


\subsection{The covering}

We are now ready to prove that for any domain $\Omega$ with $\partial \Omega \in C^{4, \alpha}$ one may find an appropriate covering by finitely many open domains that are $\varepsilon$-close in $C^{2, \gamma}$-sense to some limaçon.

Theorem 3.14 Let $\Omega, \alpha, \gamma$ and $\varepsilon$ satisfy the assumptions of Theorem 3.3 and let $R$ defined as in that Theorem. Then there exist finitely many balls $B_{j}, j \in J_{B}$ with $\bar{B}_{j} \subset \Omega$, finitely many open domains $E_{j} \subset \mathbb{R}^{2}, j \in J_{E}$, and constants $\bar{M}=\bar{M}\left(M, \rho_{\Omega}^{-1}, \varepsilon, \gamma\right)>0$ and $\delta>0$ such that:

1. $\Omega \subset \bigcup_{j \in J_{B}} B_{j} \cup \bigcup_{j \in J_{E}} E_{j}$;

2. $\left(E_{j} \cap \partial \Omega\right)^{\circ, \partial \Omega} \neq \emptyset$ for all $j \in J_{E}$;

3. every $E_{j}$ with $j \in J_{E}$ is a domain satisfying the uniform $C^{4, \alpha}$ regularity condition with constant $\bar{M}$;

4. each $E_{j}$ is $\varepsilon$-close in $C^{2, \gamma}$-sense to a limaçon $\Omega_{a, R}$ with $a \in\left[\frac{3}{16}, \frac{5}{16}\right]$.

Furthermore, for $K_{j}=\left(\bar{E}_{j} \cap \partial \Omega\right)^{\circ, \partial \Omega}$ with $j \in J_{E}$ it holds:

5. $E_{j}$ and $K_{j}$ have the same outward normal for any $x \in K_{j}$;

6. $\left\{K_{j}\right\}_{j \in J_{E}}$ is a relatively open covering of $\partial \Omega$;

7. for all $j \in J_{E}$ the diameter of $K_{j}$ is larger than $\delta$.

Proof: According to Corollary 3.7 there is a $\delta>0$ such that for every $z_{0} \in \partial \Omega$ there exists a domain $E_{z_{0}}$ such that the following holds:

- $E_{z_{0}}$ satisfies the uniform $C^{4, \alpha}$ regularity condition with constant $M_{z_{0}}=M_{z_{0}}\left(M, \rho_{\Omega}^{-1}, \varepsilon, \gamma\right)$

- $E_{z_{0}}$ is $\varepsilon$-close in $C^{2, \gamma}$-sense to a limaçon $\Omega_{a, R}$ with $a \in\left[\frac{3}{16}, \frac{5}{16}\right]$;

- letting $K_{z_{0}}$ the connected component of $\left(\bar{E}_{z_{0}} \cap \partial \Omega\right)^{\circ, \partial \Omega}$ that contains $z_{0}$, it holds

$$
B_{\delta}\left(z_{0}\right) \cap \partial \Omega=B_{\delta}\left(z_{0}\right) \cap K_{z_{0}}
$$


By compactness of $\partial \Omega$ there exist $z_{1}, \ldots, z_{N} \in \partial \Omega$ such that $\partial \Omega=$ $\bigcup_{j=1}^{N} K_{z_{j}}$. Setting $E_{j}:=E_{z_{j}}$ and $\bar{M}=\max M_{z_{j}}$ and $K_{j}$ accordingly one finds that this family $\left\{K_{j}\right\}_{j=1, \ldots, N}$ satisfies the properties of the last three items. A straightforward argument implies that $\Omega \backslash \bigcup_{j=1}^{N}\left(E_{j} \cap \Omega\right)$ can be covered by finitely open balls $B_{j}$ with $\bar{B}_{j} \subset \Omega$.

Remark 3.15 In the proof we use that $\Omega$ is simply connected. However with a slightly different argument the method would work also for general connected domains.

\section{Proving the estimates}

In this section we prove the main results of the paper. First we give pointwise estimates for the solution of (1.1), and then we prove the splitting of the solution operator between a positive singular part and a sign changing regular part.

\subsection{A maximum principle type estimate}

The pointwise estimates for the solution of (1.1) will be obtained using negative Sobolev spaces. We refer to [1, pages 62-65].

Theorem 4.1 Suppose that the hypotheses of Theorem 3.3 hold true with $0<\gamma, \alpha<1$. Then for any $q>2$ and $\varepsilon \in(0,4 R]$ there exists a constant $C>0$ with $C=C\left(\frac{1}{2-q}, M, \rho_{\Omega}^{-1}, R_{\Omega}, \varepsilon, \gamma\right)$ such that for any $f \in L^{p}(\Omega)$, with $p \in(1, \infty)$, the solution $u \in W^{4, p}(\Omega) \cap W_{0}^{2, p}(\Omega)$ of (1.1) satisfies

$$
u(x) \leq C\left(\left\|f^{+}\right\|_{L^{1}(B(x, \varepsilon) \cap \Omega)}+\|u\|_{W^{-1, q}(\Omega)}\right) \text { for every } x \in \Omega .
$$

Proof: Let $E_{j}$, with $j \in J$, be the finite covering of $\Omega$ of Theorem 3.14 and set $D_{j}:=E_{j} \cap \Omega$. We first consider the case $\varepsilon=4 R$.

Let $\psi_{i}, i \in I$, be a partition of unity with boundary associated to the covering $\left\{D_{j}\right\}_{j \in J}$ of $\Omega$ (Lemma C.15 in the appendix with $\delta=2 R$ ) such that for every $i \in I$ :

i. $\left|D^{\alpha} \psi_{i}\right| \leq c_{\alpha} R^{-|\alpha|}$ for $\alpha \in \mathbb{N}^{2}$ with $|\alpha| \leq 4$; 
ii. $\psi_{i} \neq 0$ at the boundary only if $\left(\partial E_{j(i)} \cap \partial \Omega\right)^{\circ, \partial \Omega} \neq \varnothing$.

Here $j(i)$ denotes the $j \in J$ such that $\operatorname{supp}\left(\psi_{i}\right) \subset E_{j}$. By the choice of $\psi_{i}$ it also holds that $\psi_{i} \in C_{c}^{\infty}\left(\Omega \cup\left(\partial E_{j(i)} \cap \partial \Omega\right)^{\circ, \partial \Omega}\right), \psi_{i} \in C_{c}^{\infty}\left(E_{j(i)} \cup\right.$ $\left.\left(\partial E_{j(i)} \cap \partial \Omega\right)^{\circ, \partial \Omega}\right)$ and $\psi_{i} \neq 0$ only on $\left(E_{j(i)} \cap \Omega\right) \cup\left(\partial E_{j(i)} \cap \partial \Omega\right)^{\circ, \partial \Omega}$. Notice that $I$ is a finite set.

We choose a new family of cut-off functions

$$
\chi_{i} \in C_{c}^{\infty}\left(\Omega \cup\left(\partial E_{j(i)} \cap \partial \Omega\right)^{\circ, \partial \Omega}\right) \text { for } i \in I
$$

such that for every $i \in I$ :

i. $\operatorname{supp}\left(\psi_{i}\right) \subset\left\{x \in \bar{\Omega}: \chi_{i}(x)=1\right\} \subset \operatorname{supp}\left(\chi_{i}\right) \subset$

$$
\subset\left(\left(E_{j(i)} \cap \Omega\right) \cup\left(E_{j(i)} \cap \partial \Omega\right)^{\circ, \partial \Omega}\right) \text {; }
$$

ii. $0 \leq \chi_{i}(x) \leq 1$;

iii. $\left\|\nabla^{\alpha} \chi_{i}\right\|_{\infty} \leq c_{\alpha} R^{-|\alpha|}$ for every $\alpha \in \mathbb{N}^{2}$ with $|\alpha| \leq 4$.

The functions $\chi_{i} g$ and $\psi_{i} g$ denote (with abuse of notation) respectively

$$
\chi_{i} g(x):=\left\{\begin{array}{ll}
\chi_{i}(x) g(x) & \text { in } \bar{\Omega}, \\
0 & \text { otherwise, }
\end{array} \quad \psi_{i} g(x):= \begin{cases}\psi_{i}(x) g(x) & \text { in } \bar{\Omega} \\
0 & \text { otherwise }\end{cases}\right.
$$

In the following, if not explicitly stated, every function will be extended by 0 outside its domain of definition.

Let $G_{E_{j}}$ be the Green function associated to $\Delta^{2}$ on $E_{j}$ with zero Dirichlet boundary condition. Let $v_{g, j}$ the function that satisfies

$$
\left\{\begin{aligned}
\Delta^{2} v_{g, j} & =g \text { in } E_{j} \\
v_{g, j}=\frac{\partial}{\partial \nu} v_{g, j} & =0 \text { on } \partial E_{j}
\end{aligned}\right.
$$

We define

$$
\tilde{u}_{i}(x):=\chi_{i}(x) v_{\psi_{i} f, j(i)}(x) \text { and } \tilde{u}(x):=\sum_{i \in I} \tilde{u}_{i}(x)
$$

Here $j(i)$ denotes the $j \in J$ such that $\operatorname{supp}\left(\psi_{i}\right) \subset E_{j}$. 

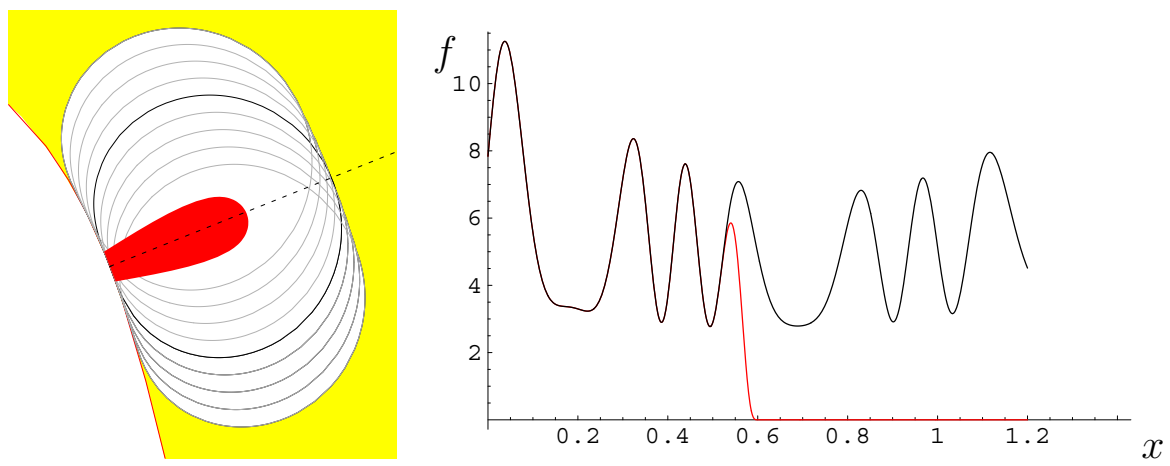

Figure 4.1 In the picture on the left one finds some $E_{j}$ 's that cover $\Omega$ locally. The dark part shows the support of the cut-off function $\psi_{i}$. On the right the effect of the multiplication with the cut-off function considered on the dashed line: in black a function $f$ and in red (lighter) the function $\psi_{i} f$. The scaling is arbitrary but consistent with the one in the following figures.

Since the Green function $G_{E_{j}}(x, y)$ is positive and bounded on $E_{j} \times E_{j}$ (Theorem 2.5) we have for some $c_{1}=c_{1}\left(M, \rho_{\Omega}^{-1}\right)$

$$
\begin{aligned}
\tilde{u}_{i}(x) & =\chi_{i}(x) \int_{E_{j(i)}} G_{E_{j(i)}}(x, y) \psi_{i}(y) f(y) d y \\
& \leq \chi_{i}(x) \int_{\operatorname{supp}\left(\psi_{i}\right) \cap \Omega} G_{E_{j(i)}}(x, y) f^{+}(y) d y \\
& \leq c_{1} \chi_{i}(x)\left\|f^{+}\right\|_{L^{1}\left(\operatorname{supp}\left(\psi_{i}\right) \cap \Omega\right)} .
\end{aligned}
$$

Notice that through the double cutting-off, both by $\psi_{i}$ and $\chi_{i}$, the influence of $f^{+}$on $\tilde{u}$ is localized, that is, with $\varepsilon_{R}:=4 R$ one gets

$$
\begin{aligned}
\tilde{u}(x) & \leq c_{1} \sum_{i \in I} \chi_{i}(x)\left\|f^{+}\right\|_{L^{1}\left(\operatorname{supp}\left(\psi_{i}\right) \cap \Omega\right)} \\
& \leq c_{2}\left\|f^{+}\right\|_{L^{1}\left(\bigcup\left\{\operatorname{supp}\left(\psi_{i}\right) \cap \Omega ; i \in I \text { with } \chi_{i}(x) \neq 0\right\}\right)} \\
& \leq c_{2}\left\|f^{+}\right\|_{L^{1}\left(B\left(x, \varepsilon_{R}\right) \cap \Omega\right)} .
\end{aligned}
$$



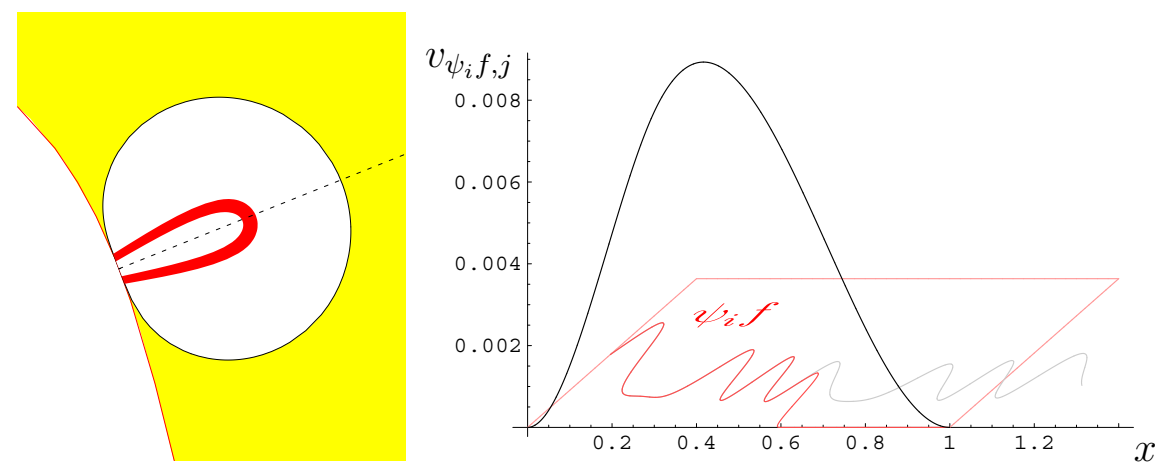

Figure 4.2 On the left one finds in black the boundary of $E_{j}$ and in red the set $\left\{x: \nabla \psi_{i}(x) \neq 0\right\}$. In the right one in black the function $v_{\psi_{i} f, j(i)}$, that is the solution of the clamped plate equation on $E_{j(i)}$ with on the right hand side $\psi_{i} f$, that is, the truncated $f$ (red in the picture).

We will now estimate the difference $u-\tilde{u}$. For every $i \in I$ one has in $E_{j(i)}$ :

$$
\Delta^{2} v_{\psi_{i} f, j(i)}=\psi_{i} \Delta^{2} u=\Delta^{2}\left(\psi_{i} u\right)-\sum_{\substack{|\alpha+\beta|=4,|\beta| \leq 3}} n_{\alpha, \beta} D^{\alpha} \psi_{i} D^{\beta} u
$$

where $n_{\alpha, \beta}$ are positive coefficients. From (4.3) we find in $E_{j}$ that

$$
\Delta^{2}\left(v_{\psi_{i} f, j(i)}-\psi_{i} u\right)=-\sum_{\substack{|\alpha+\beta|=4,|\beta| \leq 3}} n_{\alpha, \beta} D^{\alpha} \psi_{i} D^{\beta} u
$$

Furthermore the function $v_{\psi_{i} f, j(i)}-\psi_{i} u$ satisfies zero Dirichlet boundary condition on $\partial E_{j(i)}$. Indeed by construction: $u=\frac{\partial}{\partial \nu} u=0$ on $\partial E_{j(i)} \cap$ $\operatorname{supp}\left(\psi_{i}\right) \subset \partial E_{j(i)} \cap \partial \Omega$ and $\psi_{i}=\frac{\partial}{\partial \nu} \psi_{i}=0$ for $x \in \partial E_{j(i)} \backslash \operatorname{supp}\left(\psi_{i}\right)$.

Hence we may write for $x \in E_{j(i)}$

$$
v_{\psi_{i} f, j(i)}(x)=\psi_{i}(x) u(x)-R_{i}(x),
$$

where

$$
R_{i}(x):=\int_{E_{j(i)}} G_{E_{j(i)}}(x, y)\left(\Delta^{2}\left(\psi_{i}(y) u(y)\right)-\psi_{i}(y) \Delta^{2} u(y)\right) d y
$$



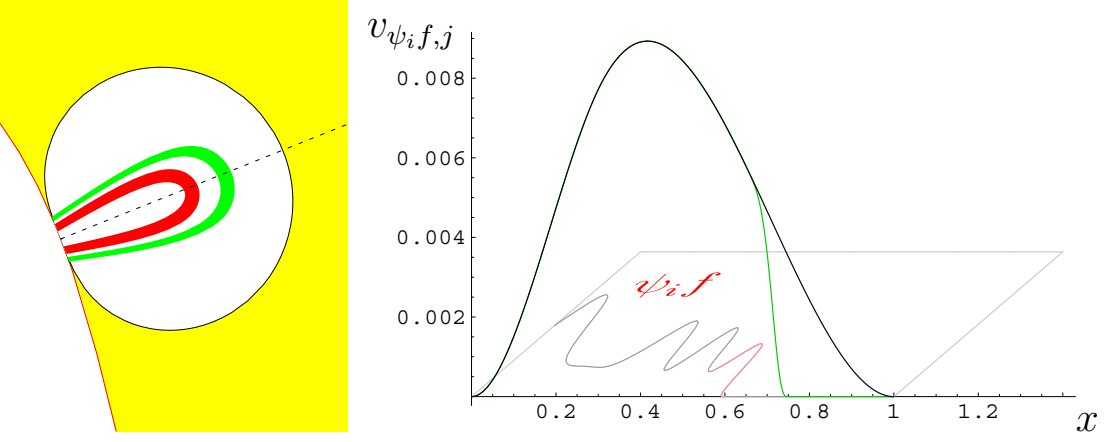

Figure 4.3 On the left one now also finds in green the set $\left\{x: \nabla \chi_{i}(x) \neq 0\right\}$. On the right in green (lighter) the function $\tilde{u}_{i}=$ $\chi_{i} v_{\psi_{i} f, j(i)}$.

On the other hand we get from (4.4)

$$
\begin{aligned}
\Delta^{2} \tilde{u}_{i} & =\Delta^{2}\left(\chi_{i} v_{\psi_{i} f, j(i)}\right) \\
& =\chi_{i} \Delta^{2} v_{\psi_{i} f, j(i)}+\left(\Delta^{2}\left(\chi_{i} v_{\psi_{i} f, j(i)}\right)-\chi_{i} \Delta^{2} v_{\psi_{i} f, j(i)}\right) \\
& =\chi_{i} \psi_{i} f+\left(\Delta^{2}\left(\chi_{i} \psi_{i} u-\chi_{i} R_{i}\right)-\chi_{i} \Delta^{2}\left(\psi_{i} u-R_{i}\right)\right) .
\end{aligned}
$$

By $\operatorname{supp}\left(\psi_{i}\right) \subset\left\{x \in \bar{\Omega}: \chi_{i}(x)=1\right\}$ we get $\Delta^{2}\left(\chi_{i} \psi_{i} u\right)=\chi_{i} \Delta^{2}\left(\psi_{i} u\right)$. Hence it holds that

$$
\Delta^{2} \tilde{u}_{i}=\chi_{i} \psi_{i} f-\Delta^{2}\left(\chi_{i} R_{i}\right)+\chi_{i} \Delta^{2} R_{i}
$$

Notice that this last relation holds in all of $\Omega$. Hence the function $\tilde{u}$ satisfies in $\Omega$

$$
\Delta^{2} \tilde{u}=f-\sum_{i \in I} \Delta^{2}\left(\chi_{i} R_{i}\right)+\sum_{i \in I} \chi_{i} \Delta^{2} R_{i} .
$$

It follows that $u-\tilde{u}$ satisfies

$$
\left\{\begin{aligned}
\Delta^{2}(u-\tilde{u}) & =\sum_{i \in I} \Delta^{2}\left(\chi_{i} R_{i}\right)-\sum_{i \in I} \chi_{i} \Delta^{2} R_{i} & & \text { in } \Omega, \\
u-\tilde{u} & =0 & & \text { on } \partial \Omega, \\
\frac{\partial}{\partial \nu}(u-\tilde{u}) & =0 & & \text { on } \partial \Omega .
\end{aligned}\right.
$$

Here we used that $\tilde{u}_{i}=\frac{\partial}{\partial \nu} \tilde{u}_{i}=0$ on $\partial \Omega$ for every $i \in I$. 
Writing

$$
\begin{aligned}
& u(x)=\tilde{u}(x)+\sum_{i \in I} \int_{\Omega} G_{\Omega}(x, y)\left(\Delta^{2}\left(\chi_{i} R_{i}\right)-\chi_{i} \Delta^{2} R_{i}\right)(y) d y \\
= & \tilde{u}(x)+\sum_{\substack{i \in I,\left|\alpha^{\prime}+\beta^{\prime}\right|=4,\left|\beta^{\prime}\right| \leq 3}} n_{\alpha^{\prime}, \beta^{\prime}} \int_{\Omega} G_{\Omega}(x, y) D^{\alpha^{\prime}} \chi_{i}(y) D^{\beta^{\prime}} R_{i}(y) d y \\
= & \tilde{u}(x)+\sum_{\substack{i \in I,|\beta|,\left|\beta^{\prime}\right| \leq 3,\left|\alpha^{\prime}+\beta^{\prime}\right|=4,|\alpha+\beta|=4}} n_{\alpha, \beta, \alpha^{\prime}, \beta^{\prime}} \int_{\Omega} G_{\Omega}(x, y) D^{\alpha^{\prime}} \chi_{i}(y) D^{\beta^{\prime}} v_{D^{\alpha} \psi_{i} D^{\beta} u, j(i)}(y) d y,
\end{aligned}
$$

and using the estimate in (4.2) we find

$$
\begin{aligned}
& u(x) \leq c_{2}\left\|f^{+}\right\|_{L^{1}\left(B\left(x, \varepsilon_{R}\right) \cap \Omega\right)} \\
& +\sum_{\substack{i \in I,|\beta|,\left|\beta^{\prime}\right| \leq 3 \\
\left|\alpha^{\prime}+\beta^{\prime}\right|=4,|\alpha+\beta|=4}} n_{\alpha, \beta, \alpha^{\prime}, \beta^{\prime}}\left\|\int_{\Omega} G_{\Omega}(\cdot, y) D^{\alpha^{\prime}} \chi_{i}(y) D^{\beta^{\prime}} v_{D^{\alpha} \psi_{i} D^{\beta} u, j(i)}(y) d y\right\|_{\infty} .
\end{aligned}
$$

In the following we will estimate the second term in the right hand side of (4.6). We fix $i \in I, \alpha, \beta, \alpha^{\prime}, \beta^{\prime} \in \mathbb{N}^{2}$ with $\left|\alpha^{\prime}+\beta^{\prime}\right|=|\alpha+\beta|=4$ and $\left|\beta^{\prime}\right|,|\beta| \leq 3$.

We first notice that it is sufficient to prove (4.1) for $q>2$ and near 2 . Indeed the result for general $q>2$ will then follow from the observation that the following inequality holds

$$
\|u\|_{W^{-1, q}(\Omega)} \leq|\Omega|^{\frac{1}{q}-\frac{1}{\tilde{q}}}\|u\|_{W^{-1, \tilde{q}(\Omega)}} \text { for any } \tilde{q}>q>2 .
$$

Let us fix $q>2$ with $q-2$ small. The Sobolev Imbedding Theorem yields that for some $c_{3}=c_{3}\left(\frac{1}{2-q}, \rho_{\Omega}^{-1}, R_{\Omega}\right)$

$$
\begin{gathered}
\left\|\int_{\Omega} G_{\Omega}(\cdot, y) D^{\alpha^{\prime}} \chi_{i}(y) D^{\beta^{\prime}} v_{D^{\alpha} \psi_{i} D^{\beta} u, j}(y) d y\right\|_{\infty} \leq \\
\leq c_{3}\left\|\int_{\Omega} G_{\Omega}(\cdot, y) D^{\alpha^{\prime}} \chi_{i}(y) D^{\beta^{\prime}} v_{D^{\alpha} \psi_{i} D^{\beta} u, j}(y) d y\right\|_{W_{0}^{1, q}(\Omega)}=\ldots
\end{gathered}
$$

Here and in the following we write simply $j$ instead of $j(i)$. 
We proceed using the regularity result for the "three-quarter weak solution" of problem (1.1) (see Definition D.15). Indeed by Theorem D.16 the solution operator from $\left(W^{3, q^{\prime}}(\Omega) \cap W_{0}^{2, q^{\prime}}(\Omega)\right)^{\prime}$ to the space $W_{0}^{1, q}(\Omega)$ is an isomorphism. Hence we get for some $c_{4}=c_{4}\left(\frac{1}{2-q}, M, \rho_{\Omega}^{-1}, R_{\Omega}\right)$

$$
\begin{aligned}
& \ldots \leq c_{4}\left\|D^{\alpha^{\prime}} \chi_{i}(\cdot) D^{\beta^{\prime}} v_{D^{\alpha} \psi_{i} D^{\beta} u, j}(\cdot)\right\|_{\left(W^{3, q^{\prime}}(\Omega) \cap W_{0}^{2, q^{\prime}}(\Omega)\right)^{\prime}} \\
&=c_{4} \sup \left\{\left\langle D^{\alpha^{\prime}} \chi_{i} D^{\beta^{\prime}} v_{D^{\alpha} \psi_{i} D^{\beta} u, j}, \varphi\right\rangle \mid \varphi \in W^{3, q^{\prime}}(\Omega) \cap W_{0}^{2, q^{\prime}}(\Omega)\right. \\
&\text { with } \left.\|\varphi\|_{W^{3, q^{\prime}}(\Omega)} \leq 1\right\}=\ldots
\end{aligned}
$$

Notice that the constant in Theorem D.16 depends on $q$ and $q^{\prime}$. However, since we consider $q$ near 2 we can choose a constant that depends only on the distance of $q$ to 2 .

Next, we consider a restriction from $\left(W^{3, q^{\prime}}(\Omega) \cap W_{0}^{2, q^{\prime}}(\Omega)\right)^{\prime}$ to $\left(W^{3, q^{\prime}}\left(E_{j}\right) \cap W_{0}^{2, q^{\prime}}\left(E_{j}\right)\right)^{\prime}$. One uses that the cut-off function $\chi_{i}$ has support in $\left(E_{j} \cap \Omega\right) \cup\left(\partial \Omega \cap \partial E_{j}\right)^{\circ, \partial \Omega}$. Proceeding formally we take a cut-off function $h_{i} \in C_{c}^{\infty}\left(\Omega \cup\left(\partial \Omega \cap \partial E_{j}\right)^{\circ, \partial \Omega}\right)$ such that:

i. $\operatorname{supp}\left(\chi_{i}\right) \subset\left\{x \in \bar{\Omega}: h_{i}(x)=1\right\}$;

ii. $\operatorname{supp}\left(h_{i}\right) \subset\left(E_{j} \cap \Omega\right) \cup\left(\partial E_{j} \cap \partial \Omega\right)^{\circ, \partial \Omega}$;

iii. $0 \leq h_{i} \leq 1$;

iv. $\left\|\nabla^{\alpha} h_{i}\right\|_{\infty} \leq c_{\alpha} R^{-|\alpha|}$ for every $\alpha \in \mathbb{N}^{2}$ with $|\alpha| \leq 4$.

Since $\operatorname{supp}\left(\chi_{i}\right) \subset\left(E_{j} \cap \Omega\right) \cup\left(\partial E_{j} \cap \partial \Omega\right)^{\circ, \partial \Omega}$ such a cut-off function exists. The function $h_{i} \varphi$ lies in $W^{3, q^{\prime}}\left(E_{j}\right) \cap W_{0}^{2, q^{\prime}}\left(E_{j}\right)$ for every $\varphi \in$ $W^{3, q^{\prime}}(\Omega) \cap W_{0}^{2, q^{\prime}}(\Omega)$ and moreover it holds

$$
\begin{aligned}
\left\langle D^{\alpha^{\prime}} \chi_{i} D^{\beta^{\prime}} v_{D^{\alpha} \psi_{i} D^{\beta} u, j}, \varphi\right\rangle_{\Omega} & =\left\langle D^{\alpha^{\prime}} \chi_{i} D^{\beta^{\prime}} v_{D^{\alpha} \psi_{i} D^{\beta} u, j}, h_{i} \varphi\right\rangle_{\Omega} \\
& =\left\langle D^{\alpha^{\prime}} \chi_{i} D^{\beta^{\prime}} v_{D^{\alpha} \psi_{i} D^{\beta} u, j}, h_{i} \varphi\right\rangle_{E j} .
\end{aligned}
$$


Since there exists $c_{5}>0$ such that $\left\|h_{i} \varphi\right\|_{W^{3, q^{\prime}}(\Omega)} \leq c_{5} R^{-3}\|\varphi\|_{W^{3, q^{\prime}}(\Omega)}$ we get

$$
\begin{array}{r}
\ldots=c_{4} \sup \left\{\left\langle D^{\alpha^{\prime}} \chi_{i} D^{\beta^{\prime}} v_{D^{\alpha} \psi_{i} D^{\beta} u, j}, h_{i} \varphi\right\rangle_{E_{j}} \mid \varphi \in W^{3, q^{\prime}}(\Omega) \cap W_{0}^{2, q^{\prime}}(\Omega)\right. \\
\text { with } \left.\left\|h_{i} \varphi\right\|_{W^{3, q^{\prime}}(\Omega)} \leq c_{5} R^{-3}\right\} \\
\leq c_{4} \sup \left\{\left\langle D^{\alpha^{\prime}} \chi_{i} D^{\beta^{\prime}} v_{D^{\alpha} \psi_{i} D^{\beta} u, j}, \tilde{\varphi}\right\rangle_{E_{j}} \mid \tilde{\varphi} \in W^{3, q^{\prime}}\left(E_{j}\right) \cap W_{0}^{2, q^{\prime}}\left(E_{j}\right)\right. \\
\text { with } \left.\|\tilde{\varphi}\|_{W^{3, q^{\prime}}\left(E_{j}\right)} \leq c_{5} R^{-3}\right\} \\
\leq c_{6} \sup \left\{\left\langle D^{\beta^{\prime}} v_{D^{\alpha} \psi_{i} D^{\beta} u, j}, \tilde{\varphi}\right\rangle_{E_{j}} \mid \tilde{\varphi} \in W^{3, q^{\prime}}\left(E_{j}\right) \cap W_{0}^{2, q^{\prime}}\left(E_{j}\right)\right. \\
\text { with } \left.\|\tilde{\varphi}\|_{W^{3, q^{\prime}}\left(E_{j}\right)} \leq 1\right\}=\ldots
\end{array}
$$

Here $c_{6}=c_{6}\left(\frac{1}{2-q}, M, \rho_{\Omega}^{-1}, R_{\Omega}\right)$ since $R$ depends on $M$.

We now proceed by integrating by parts. Since $v_{D^{\alpha} \psi_{i} D^{\beta} u, j}$ and $\tilde{\varphi}$ and their first derivatives are zero on $\partial E_{j}$ there is no contribution from the boundary. We find

$$
\begin{aligned}
& \ldots=c_{6} \sup \left\{\left\langle v_{D^{\alpha} \psi_{i} D^{\beta} u, j}, D^{\beta^{\prime}} \tilde{\varphi}\right\rangle_{E_{j}} \mid \tilde{\varphi} \in W^{3, q^{\prime}}\left(E_{j}\right) \cap W_{0}^{2, q^{\prime}}\left(E_{j}\right)\right. \\
&\text { with } \left.\|\tilde{\varphi}\|_{W^{3, q^{\prime}}\left(E_{j}\right)} \leq 1\right\} \\
& \leq c_{6} \sup \left\{\left\langle v_{D^{\alpha} \psi_{i} D^{\beta} u, j}, \varphi\right\rangle_{E_{j}} \mid \varphi \in W^{3-\left|\beta^{\prime}\right|, q^{\prime}}\left(E_{j}\right) \cap\right. \\
&\left.\cap W_{0}^{\min \left\{2,3-\left|\beta^{\prime}\right|\right\}, q^{\prime}}\left(E_{j}\right),\|\varphi\|_{W^{3-\left|\beta^{\prime}\right|, q^{\prime}}\left(E_{j}\right)} \leq 1\right\} \\
& \leq c_{6} \sup \left\{\left\langle v_{D^{\alpha} \psi_{i} D^{\beta} u, j}, \varphi\right\rangle_{E_{j}} \mid \varphi \in L^{q^{\prime}}\left(E_{j}\right),\|\varphi\|_{L^{q^{\prime}\left(E_{j}\right)}} \leq 1\right\} \\
&=c_{6}\left\|v_{D^{\alpha} \psi_{i} D^{\beta} u, j}\right\|_{L^{q}\left(E_{j}\right)}=\ldots
\end{aligned}
$$

Next, we apply the regularity result for weak solution of problem (1.1) (see Definition D.12). Notice that in order to do that one needs that $\partial E_{j} \in C^{4, \alpha}$. By the result in Theorem D.13 we get for some $c_{7}=$ $c_{7}\left(\frac{1}{2-q}, M, \rho_{\Omega}^{-1}, R_{\Omega}\right)$

$$
\begin{aligned}
\ldots & \leq c_{7}\left\|D^{\alpha} \psi_{i} D^{\beta} u\right\|_{\left(W^{4, q^{\prime}}\left(E_{j}\right) \cap W_{0}^{2, q^{\prime}}\left(E_{j}\right)\right)^{\prime}} \\
& =c_{7} \sup \left\{\left\langle D^{\alpha} \psi_{i} D^{\beta} u, \varphi\right\rangle_{E_{j}} \mid \varphi \in W^{4, q^{\prime}}\left(E_{j}\right) \cap W_{0}^{2, q^{\prime}}\left(E_{j}\right),\right. \\
& \left.\|\varphi\|_{W^{4, q^{\prime}\left(E_{j}\right)}} \leq 1\right\}
\end{aligned}
$$


Since we consider $q$ near 2 we can choose the dependance on $q$ of the form $\frac{1}{2-q}$ in the constant that appears in the estimate of Theorem D.13.

We now consider an extension from $\left(W^{4, q^{\prime}}\left(E_{j}\right) \cap W_{0}^{2, q^{\prime}}\left(E_{j}\right)\right)^{\prime}$ to $\left(W^{4, q^{\prime}}(\Omega) \cap W_{0}^{2, q^{\prime}}(\Omega)\right)^{\prime}$. Since $\psi_{i}$ has compact support in $\left(\Omega \cap E_{j}\right) \cup$ $\left(\partial \Omega \cap \partial E_{j}\right)^{\circ, \partial \Omega}$ one has

$$
\left\langle D^{\alpha} \psi_{i} D^{\beta} u, \varphi\right\rangle_{E_{j}}=\left\langle D^{\alpha} \psi_{i} D^{\beta} u, \varphi\right\rangle_{\Omega},
$$

which implies

$$
\begin{aligned}
\ldots & =c_{7} \sup \left\{\left\langle D^{\alpha} \psi_{i} D^{\beta} u, \varphi\right\rangle_{\Omega} \mid \varphi \in W^{4, q^{\prime}}\left(E_{j}\right) \cap W_{0}^{2, q^{\prime}}\left(E_{j}\right),\right. \\
& \left.\|\varphi\|_{W^{4, q^{\prime}}\left(E_{j}\right)} \leq 1\right\} \\
\leq & c_{7} \sup \left\{\left\langle D^{\alpha} \psi_{i} D^{\beta} u, \varphi\right\rangle_{\Omega} \mid \varphi \in W^{4, q^{\prime}}(\Omega) \cap W_{0}^{2, q^{\prime}}(\Omega),\right. \\
\left.\|\varphi\|_{W^{4, q^{\prime}}(\Omega)} \leq 1\right\} & \\
& \leq c_{8} \sup \left\{\left\langle D^{\beta} u, \varphi\right\rangle_{\Omega} \mid \varphi \in W^{4, q^{\prime}}(\Omega) \cap W_{0}^{2, q^{\prime}}(\Omega),\|\varphi\|_{W^{4, q^{\prime}}(\Omega)} \leq 1\right\} .
\end{aligned}
$$

Here $c_{8}=c_{8}\left(\frac{1}{2-q}, M, \rho_{\Omega}^{-1}, R_{\Omega}\right)$.

The last step is an integration by part. We do not have any contribution from the boundary since $u$ and $\varphi$ and their first derivative are zero on $\partial \Omega$. Hence one finds

$$
\begin{aligned}
\ldots & =c_{8} \sup \left\{\left\langle u, D^{\beta} \varphi\right\rangle_{\Omega} \mid \varphi \in W^{4, q^{\prime}}(\Omega) \cap W_{0}^{2, q^{\prime}}(\Omega),\|\varphi\|_{W^{4, q^{\prime}}(\Omega)} \leq 1\right\} \\
& \leq c_{8} \sup \left\{\langle u, \tilde{\varphi}\rangle_{\Omega} \mid \tilde{\varphi} \in W^{4-|\beta|, q^{\prime}}(\Omega) \cap W_{0}^{\min \{2,4-|\beta|\}, q^{\prime}}(\Omega),\right. \\
& \leq c_{8} \sup \left\{\left\langle u \|_{W^{4-|\beta|, q^{\prime}}(\Omega)} \leq 1\right\}\right. \\
& =c_{8}\|u\|_{\left(W_{0}^{1, q^{\prime}}(\Omega)\right)^{\prime}} .
\end{aligned}
$$

The claim follows for $\varepsilon_{R}=4 R$. For $\varepsilon \in\left(0, \varepsilon_{R}\right]$ one may repeat the same construction with a refinement of the partition of unity $\psi_{i}, i \in I$. 
Remark 4.2 The hypothesis $\Omega$ simply connected is required in order to use Theorem D.16. The result can be proved also for general connected domains using a generalization of Theorem D.16.

\subsection{Green function estimates}

In this section we prove Theorem 1.1 and we give optimal estimates from below for the Green function of a two-dimensional domain $\Omega$ with $\partial \Omega \in$ $C^{16}$. In this section we have to assume more regularity on the boundary of $\Omega$ in order to use [8, Th.2.6]. As before, $G_{\Omega}$ denotes the Green function associated to problem (1.1) on $\Omega$.

We first present some preliminary lemmas.

Lemma 4.3 Assume that $\Omega$ is a bounded domain in $\mathbb{R}^{2}$ with $\partial \Omega \in C^{16}$. Then

$$
G_{\Omega} \in W^{3, p}\left(\Omega^{2}\right) \text { for any } p \in[1,2) \text {. }
$$

Proof: In [8] one finds for any $\beta \in \mathbb{N}^{2}$ with $|\beta| \leq 3$

$$
\left|D^{\beta} G_{\Omega}(x, y)\right| \preceq|x-y|^{-1} \min \left\{1, \frac{d(y)}{|x-y|}\right\}^{2} .
$$

The result follows directly from (4.7).

Lemma 4.4 Let $\Omega$ be a bounded domain in $\mathbb{R}^{2}$ with $\partial \Omega \in C^{16}$. Then for every $\gamma \in(0,1)$

$$
G_{\Omega} \in C^{15, \gamma}\left(\left\{(x, y) \in \bar{\Omega}^{2}: x \neq y\right\}\right) \text { and } G_{\Omega} \in C^{1, \gamma}\left(\bar{\Omega}^{2}\right) \cap C_{0}^{1}\left(\bar{\Omega}^{2}\right) .
$$

Proof: From general regularity results for elliptic partial differential equations (see [2]) it is well known that $G_{\Omega} \in C^{15, \gamma}\left(\left\{(x, y) \in \bar{\Omega}^{2}: x \neq y\right\}\right)$ for any $\gamma \in(0,1)$. Indeed, in general, given $l \in \mathbb{N}, \beta \in[0,1)$ and a bounded domain $\mathcal{D} \in C^{l, \beta}$ then the regularity of $G_{\mathcal{D}}$ on $\left\{(x, y) \in \overline{\mathcal{D}}^{2}: x \neq y\right\}$ is as follows:

$$
\begin{aligned}
& \text { if } \beta=0: G_{\mathcal{D}} \in C^{l-1, \gamma}\left(\left\{(x, y) \in \overline{\mathcal{D}}^{2}: x \neq y\right\}\right) \text { for any } \gamma \in(0,1) \\
& \text { if } \beta \neq 0: G_{\mathcal{D}} \in C^{l, \beta}\left(\left\{(x, y) \in \overline{\mathcal{D}}^{2}: x \neq y\right\}\right) \text {. }
\end{aligned}
$$


The result that $G_{\Omega} \in C^{1, \gamma}\left(\bar{\Omega}^{2}\right)$ follows directly from Lemma 4.3 via the Sobolev imbedding Theorem ([1, Th.4.12 Part 2]). Hence $G_{\Omega} \in$ $W^{3, p}\left(\Omega^{2}\right) \cap C^{1, \gamma}\left(\bar{\Omega}^{2}\right)$ for $p \in[1,2)$ and $\gamma \in(0,1)$. Moreover the function and its first derivatives are zero on $\partial \Omega \times \Omega$ and on $\Omega \times \partial \Omega$. Hence, by continuity and Theorem IX.17 in [4] it follows that $G_{\Omega} \in C_{0}^{1}\left(\bar{\Omega}^{2}\right)$ (and also $G_{\Omega} \in W_{0}^{2, p}\left(\Omega^{2}\right)$ for $p \in[1,2)$ ).

Proof of Theorem 1.1: Following the construction in Theorem 4.1, see (4.5), one may write the solution of problem (1.1) as

$$
\begin{aligned}
u(x)= & \tilde{u}(x)+\int_{\Omega} G_{\Omega}(x, z) \sum_{i \in I}\left(\Delta^{2}\left(\chi_{i}(z) R_{i}(z)\right)-\chi_{i}(z) \Delta^{2} R_{i}(z)\right) d z \\
= & \sum_{i \in I} \chi_{i}(x) \int_{E_{j(i)}} G_{E_{j(i)}}(x, z) \psi_{i}(z) f(z) d z+ \\
& +\int_{\Omega} G_{\Omega}(x, z) \sum_{i \in I}\left(\Delta^{2}\left(\chi_{i}(z) R_{i}(z)\right)-\chi_{i}(z) \Delta^{2} R_{i}(z)\right) d z,
\end{aligned}
$$

where

$$
R_{i}(z)=\int_{E_{j(i)}} G_{E_{j(i)}}\left(z, z^{\prime}\right)\left(\Delta^{2}\left(\psi_{i}\left(z^{\prime}\right) u\left(z^{\prime}\right)\right)-\psi_{i}\left(z^{\prime}\right) \Delta^{2} u\left(z^{\prime}\right)\right) d z^{\prime},
$$

and $j(i)$ denotes the $j \in J$ such that $\operatorname{supp}\left(\psi_{i}\right) \subset E_{j}$. Considering formally $f(x)=\delta_{y}(x)$ we get

$$
\begin{aligned}
G_{\Omega}(x, y) & =\sum_{i \in I} \chi_{i}(x) G_{E_{j(i)}}(x, y) \psi_{i}(y)+ \\
+ & \int_{\Omega} G_{\Omega}(x, z) \sum_{i \in I}\left(\Delta^{2}\left(\chi_{i}(z) R_{i}(z, y)\right)-\chi_{i}(z) \Delta^{2} R_{i}(z, y)\right) d z
\end{aligned}
$$

where $R_{i}(z, y)=$

$$
\int_{E_{j(i)}} G_{E_{j(i)}}\left(z, z^{\prime}\right)\left(\Delta^{2}\left(\psi_{i}\left(z^{\prime}\right) G_{\Omega}\left(z^{\prime}, y\right)\right)-\psi_{i}\left(z^{\prime}\right) \Delta^{2} G_{\Omega}\left(z^{\prime}, y\right)\right) d z^{\prime} .
$$

We define

$$
\begin{aligned}
& G_{\Omega}^{\mathrm{sing}}(x, y):=\sum_{i \in I} \chi_{i}(x) G_{E_{j}}(x, y) \psi_{i}(y) \\
& G_{\Omega}^{\mathrm{reg}}(x, y):=G_{\Omega}(x, y)-G_{\Omega}^{\mathrm{sing}}(x, y)
\end{aligned}
$$


From the definition it follows that $G_{\Omega}^{\text {reg }} \in C^{15, \gamma}\left(\bar{\Omega}^{2}\right)$ for any $\gamma \in(0,1)$. Indeed, writing explicitly $R_{i}$ and looking at the support of the term inside the integral, we find

$$
\begin{aligned}
& G_{\Omega}^{\mathrm{reg}}(x, y)= \\
& =\sum_{i \in I} \sum_{\substack{|\alpha+\beta|=4,\left|\alpha^{\prime}+\beta^{\prime}\right|=4,|\beta|,\left|\beta^{\prime}\right| \leq 3}} n_{\alpha, \beta, \alpha^{\prime}, \beta^{\prime}} \int_{B_{i}} G_{\Omega}(x, z) D^{\alpha} \chi_{i}(z) \text {. } \\
& \cdot\left(D^{\beta} \int_{A_{i}} G_{E_{j(i)}}\left(z, z^{\prime}\right) D^{\alpha^{\prime}} \psi_{i}\left(z^{\prime}\right) D^{\beta^{\prime}} G_{\Omega}\left(z^{\prime}, y\right) d z^{\prime}\right) d z,
\end{aligned}
$$

with $n_{\alpha, \beta, \alpha^{\prime}, \beta^{\prime}}$ some positive coefficients and

$$
B_{i}=\left\{z \in \Omega: \nabla \chi_{i}(z) \neq 0\right\} \text { and } A_{i}=\left\{z \in \Omega: \nabla \psi_{i}(z) \neq 0\right\} .
$$

Since $A_{i} \cap B_{i}=\varnothing$ one always has $z \neq z^{\prime}$ in (4.10). Hence $G_{E_{j}}\left(z, z^{\prime}\right) \in$ $C^{\infty}\left(B_{j} \times A_{j}\right)$. Since the term $D^{\alpha^{\prime}} \psi_{i}\left(z^{\prime}\right) D^{\beta^{\prime}} G_{\Omega}\left(z^{\prime}, y\right)$ is integrable it follows that $G_{\Omega}^{\text {reg }}$ is as regular as we want in the interior. The regularity up to the boundary is given by the fact that $\partial \Omega \in C^{16}$.

The positivity of $G_{\Omega}^{\text {sing }}$ follows from the positivity of $G_{E_{j}}$. Furthermore by Lemma 4.4, the definition of $G_{\Omega}^{\text {sing }}$ and since $G_{\Omega}^{\text {reg }} \in C^{15, \gamma}\left(\bar{\Omega}^{2}\right)$ for any $\gamma \in(0,1)$ holds, it follows that $G_{\Omega}^{\text {sing }} \in C^{1, \gamma}\left(\bar{\Omega}^{2}\right) \cap C_{0}^{1}\left(\bar{\Omega}^{2}\right)$ and moreover that $G_{\Omega}^{\text {sing }} \in C^{15, \gamma}\left(\left\{(x, y) \in \bar{\Omega}^{2}: x \neq y\right\}\right)$ for any $\gamma \in(0,1)$. Notice that by the boundary condition satisfied by $G_{\Omega}$ and $G_{\Omega}^{\text {sing }}$ we also have that $G_{\Omega}^{\text {reg }} \in C_{0}^{1}\left(\bar{\Omega}^{2}\right)$.

Remark 4.5 The functions $G_{\Omega}^{\text {reg }}$ and $G_{\Omega}^{\text {sing }}$ defined in the proof of Theorem 1.1 are not yet symmetric. In order to get symmetric functions one may consider $G_{\Omega, \text { new }}^{\cdots}(x, y):=\frac{1}{2} G_{\Omega}^{\cdots}(x, y)+\frac{1}{2} G_{\Omega}^{\cdots}(y, x)$.

Optimal estimates from above for the Green function as well as estimates for the absolute value are known. We refer to [18], [13] and [8]. We will next prove optimal estimates from below for $G_{\Omega}$.

First we prove the following lemma.

Lemma 4.6 Let $\Omega$ be a bounded domain in $\mathbb{R}^{2}$ with $\partial \Omega \in C^{16}$. Then $G_{\Omega}$ satisfies

$$
\left\|\nabla G_{\Omega}(\cdot, y)\right\|_{L^{p}(\Omega)} \leq c_{p, \Omega}^{\prime} d(y)^{2} \text { for every } y \in \Omega \text { and } p \in[1,2) .
$$


Proof: Via [8, Th.2.6] one finds $\left\|\nabla G_{\Omega}(\cdot, y)\right\|_{L^{p}(\Omega)}^{p} \leq$

$$
\begin{aligned}
& \leq c_{\Omega} \int_{\Omega} d(y)^{p} \min \left\{1, \frac{d(y)}{|x-y|}\right\}^{p} \min \left\{1, \frac{d(x)}{|x-y|}\right\}^{p} d x \\
& \leq c_{\Omega} d(y)^{2 p} \int_{\Omega} \frac{1}{|x-y|^{p}} d x \leq c_{p, \Omega}^{\prime} d(y)^{2 p},
\end{aligned}
$$

for $p \in[1,2)$.

Now we may prove the estimate for $G_{\Omega}(x, y)$ from below that was stated in Theorem 1.5.

Proof of Theorem 1.5: Since $G_{\Omega}(x, y)=G_{\Omega}^{\text {sing }}(x, y)+G_{\Omega}^{\text {reg }}(x, y)$, with $G_{\Omega}^{\text {sing }}$ and $G_{\Omega}^{\text {reg }}$ defined in (4.8) and (4.9) respectively, and $G_{\Omega}^{\text {sing }}$ is positive it holds

$$
G_{\Omega}(x, y) \geq-\left|G_{\Omega}^{\mathrm{reg}}(x, y)\right| \text { for every } x, y \in \Omega .
$$

Hence in order to prove the result it is sufficient to get an estimate of the absolute value of $G_{\Omega}^{\text {reg }}$.

We first study the $W^{4, p}$-norm of $G_{\Omega}^{\text {reg }}(\cdot, y)$ for $p \in(1, \infty)$.

Let $A_{i}$ and $B_{i}$ as defined in (4.11). From (4.10) and elliptic regularity theory (see Theorem D.6) it follows that

$$
\left\|G_{\Omega}^{\mathrm{reg}}(\cdot, y)\right\|_{W^{4, p}(\Omega)} \leq c \sum_{i \in I} \sum_{\substack{|\alpha+\beta|=4,|\beta| \leq 3}} n_{\alpha, \beta}\left\|D^{\alpha} \chi_{i}(\cdot) D^{\beta} R_{i}(\cdot, y)\right\|_{L^{p}\left(B_{i}\right)} .
$$

We study separately the term $\left\|D^{\alpha} \chi_{i}(\cdot) D^{\beta} R_{i}(\cdot, y)\right\|_{L^{p}\left(B_{i}\right)}$. One has

$$
\begin{aligned}
& \left\|D^{\alpha} \chi_{i}(\cdot) D^{\beta} R_{i}(\cdot, y)\right\|_{L^{p}\left(B_{i}\right)} \\
\leq & c_{\Omega} \sum_{\substack{\left|\alpha^{\prime}+\beta^{\prime}\right|=4 \\
\left|\beta^{\prime}\right| \leq 3}} n_{\alpha^{\prime}, \beta^{\prime}}\left\|D^{\beta} \int_{A_{i}} G_{E_{j}}\left(\cdot, z^{\prime}\right) D^{\alpha^{\prime}} \psi_{i}\left(z^{\prime}\right) D^{\beta^{\prime}} G_{\Omega}\left(z^{\prime}, y\right) d z^{\prime}\right\|_{L^{p}\left(B_{i}\right)} .
\end{aligned}
$$

We first observe that $G_{E_{j}}$ is non singular in $B_{i} \times A_{i}$. Indeed since $\bar{A}_{i} \cap$ $\bar{B}_{i}=\varnothing$, the function $G_{E_{j}}\left(z, z^{\prime}\right)$ is in $C^{\infty}\left(B_{j} \times A_{j}\right)$ and all its derivatives are bounded by a constant depending only on $\Omega$.

The next step consists in an integration by part. There are no contribution from the boundary since in $\partial A_{i} \cap \Omega$ the function $\psi_{i}$ and its derivatives 
are zero, while in $\partial A_{i} \cap \partial \Omega$ both $G_{E_{j}}$ and $G_{\Omega}$ and their first derivatives are zero.

Let $\beta^{\prime \prime} \in \mathbb{N}^{2}$ denote a multi-index such that $\beta^{\prime \prime}<\beta^{\prime},\left|\beta^{\prime \prime}\right|=\left|\beta^{\prime}\right|-1$. We obtain

$$
\begin{aligned}
& \left\|D^{\alpha} \chi_{i}(\cdot) D^{\beta} R_{i}(\cdot, y)\right\|_{L^{p}\left(B_{i}\right)} \leq c_{\Omega} \sum_{\substack{\left|\alpha^{\prime}+\beta^{\prime}\right|=4,\left|\beta^{\prime}\right| \leq 3}} \ldots \\
& n_{\alpha^{\prime}, \beta^{\prime}}\left\|D^{\beta} \int_{A_{i}} D^{\beta^{\prime \prime}}\left(G_{E_{j}}\left(\cdot, z^{\prime}\right) D^{\alpha^{\prime}} \psi_{i}\left(z^{\prime}\right)\right) D^{\beta^{\prime}-\beta^{\prime \prime}} G_{\Omega}\left(z^{\prime}, y\right) d z^{\prime}\right\|_{L^{p}\left(B_{i}\right)} \\
& \leq c_{\Omega, p} \sum_{\substack{\left|\alpha^{\prime}+\beta^{\prime}\right|=4,\left|\beta^{\prime}\right|=2,3}} n_{\alpha^{\prime}, \beta^{\prime}} \int_{\Omega}\left|D^{\beta^{\prime}-\beta^{\prime \prime}} G_{\Omega}\left(z^{\prime}, y\right)\right| d z^{\prime} \leq c_{\Omega, p}^{\prime} d(y)^{2} .
\end{aligned}
$$

In the last step we used Lemma 4.6.

Since $G_{\Omega}^{\mathrm{reg}}(x, y) \in W^{4, p}(\Omega) \cap W_{0}^{2, p}(\Omega)$ for any $p \in(1, \infty)$, from [6, Lemma 5] it follows that

$$
\frac{\left|G_{\Omega}^{\mathrm{reg}}(x, y)\right|}{d(x)^{2}} \leq c_{\Omega}\left\|G_{\Omega}^{\mathrm{reg}}(\cdot, y)\right\|_{W^{4, p}(\Omega)} .
$$

Hence we obtain

$$
\left|G_{\Omega}^{\mathrm{reg}}(x, y)\right| \leq c_{\Omega}^{\prime \prime} d(y)^{2} d(x)^{2} .
$$

The claim follows.

Remark 4.7 In [6, Lemma 5] the authors consider a bounded domain $\Omega$ with $\partial \Omega$ smooth. One can consider a weaker assumption on the boundary. Indeed, in order to apply the Rellich-Kondrachov Theorem, [1, Th.6.3], it is sufficient that $\Omega$ is bounded and satisfies the strong Lipschitz condition, [1, Def.4.9]. Notice that if $\Omega$ satisfies the uniform $C^{l}$ regularity condition with $l \geq 2$ then $\Omega$ satisfies also the strong Lipschitz condition.

\section{Appendices}

\section{A Improved $\varepsilon$-closeness to the disk}

In [12, Prop.2.6] the authors show that $C^{2 m, \gamma}$-closeness to the disk (Definition 2.4) implies the existence of a conformal map that satisfies the 
$C^{2 m-1}$-closeness condition. This result can be improved. Indeed, Sassone recently showed in [22] that from $C^{2 m, \gamma}$-closeness to the disk one gets the existence of a conformal map that also satisfies the $C^{2 m, \gamma}$-closeness condition. We state the result in the following proposition.

Proposition A.1 ([22]) Let $\gamma \in(0,1)$ and $m \in \mathbb{N}$ be given. For all $\delta>0$ there exist $\varepsilon_{0}=\varepsilon_{0}(\delta, m, \gamma)>0$ such that for all $\varepsilon \in\left[0, \varepsilon_{0}\right]$ we have the following.

If $\Omega$ is $\varepsilon$-close in $C^{2 m, \gamma}$-sense to the disk $B$, then there exists a biholomorphic mapping $\varphi: \bar{B} \rightarrow \bar{\Omega}$, with $\varphi \in C^{2 m, \gamma}(\bar{B})$ and $\varphi^{-1} \in C^{2 m, \gamma}(\bar{\Omega})$, such that

$$
\|\varphi-I d\|_{C^{2 m, \gamma}(\bar{B})} \leq \delta .
$$

\section{B Previously known estimates for $G_{\Omega_{a}}$}

For easy reference we recall here some results from [7] and [8].

Proposition B.1 For every limaçon $\Omega_{a}$ with $a \in[0, \bar{a}]$ the following twosided estimate holds for $(x, y) \in \Omega_{a}^{2}$ :

$$
G_{\Omega_{a}}(x, y) \sim d_{\Omega_{a}}(x) d_{\Omega_{a}}(y) \min \left\{1, \frac{d_{\Omega_{a}}(x) d_{\Omega_{a}}(y)}{|x-y|^{2}}\right\} .
$$

Proposition B.2 Let $k \in \mathbb{N}^{2}$ with $1 \leq|k| \leq 3$. For every limaçon $\Omega_{a}$ with $a \in[0, \bar{a}]$ the following estimates hold for $x, y \in \Omega_{a}$ :

$$
\begin{gathered}
\text { when }|k|=3:\left|D_{x}^{k} G_{\Omega_{a}}(x, y)\right| \preceq|x-y|^{-1} \min \left\{1, \frac{d_{\Omega_{a}}(y)}{|x-y|}\right\}^{2}, \\
\text { when }|k|=2:\left|D_{x}^{k} G_{\Omega_{a}}(x, y)\right| \preceq \log \left(1+\frac{d_{\Omega_{a}}(y)^{2}}{|x-y|^{2}}\right) \\
\sim \log \left(2+\frac{d_{\Omega_{a}}(y)}{|x-y|}\right) \min \left\{1, \frac{d_{\Omega_{a}}(y)}{|x-y|}\right\}^{2}, \\
\text { when }|k|=1:\left|D_{x}^{k} G_{\Omega_{a}}(x, y)\right| \preceq d_{\Omega_{a}}(y) \min \left\{1, \frac{d_{\Omega_{a}}(x) d_{\Omega_{a}}(y)}{|x-y|^{2}}\right\} .
\end{gathered}
$$

Remark B.3 We refer to [8, Th.2.6] for estimates of the derivatives of the Green function associated to polyharmonic Dirichlet boundary value problems in domains $\Omega \subset \mathbb{R}^{n}$ with $n \geq 2$. There it is assumed that $\Omega$ is bounded and that $\partial \Omega \in C^{r}$ with $r>4 m+2$. 


\section{Technical lemmas}

In this section we give the proof of some results needed in the proof of Theorem 3.3. We assume the same hypothesis and we use the same notation as in the proof of this theorem. In particular, we recall that the domain $\Omega$ satisfies the uniform $C^{4, \alpha}$ regularity condition with constant $M$.

\section{C.1 The mapping is close to identity}

\section{C.1.1 Proof of Proposition 3.10}

In the following lemma we give the effect on the norms of the scaling defined in (3.1).

Lemma C.1 Let $\Omega$ be a subset of $\mathbb{R}^{n}$ and let $f: \bar{\Omega} \rightarrow \bar{\Omega}^{\prime}$ be a $C^{2, \gamma_{-}}$ function. Let $f^{R}$ be the $f$ scaled as in (3.1). Then it holds

$$
\begin{array}{r}
\left\|f^{R}-I d\right\|_{C^{2, \gamma}\left(R^{-1} \bar{\Omega}\right)}=\frac{1}{R}\|f-I d\|_{C^{0}(\bar{\Omega})}+\sum_{i=1}^{n}\left\|\frac{\partial}{\partial x_{i}}(f-I d)\right\|_{C^{0}(\bar{\Omega})} \\
+R \sum_{i, j=1}^{n}\left\|\frac{\partial^{2}}{\partial x_{i} x_{j}} f\right\|_{C^{0}(\bar{\Omega})}+R^{1+\gamma} \sum_{i, j=1}^{n}\left[\frac{\partial^{2}}{\partial x_{i} x_{j}} f\right]_{C^{\gamma}(\bar{\Omega})}(\text { C. } 1)
\end{array}
$$

The proof is obvious and will be skipped.

Proof of Proposition 3.10: We estimate separately the terms in the righthand side of (C.1) for $f=f_{a, R}$ and $\Omega=\Omega_{a, R}$.

1. Since $-y \leq R$ and $k_{a, R}-g_{\delta} \equiv 0$ for $|x| \in\left[R x_{a}^{*}, R x_{a}\right]$ we find

$$
\left\|f_{a, R}-I d\right\|_{C^{0}\left(\bar{\Omega}_{a, R}\right)} \leq 4 R\left\|\frac{k_{a, R}-g_{\delta}}{3 R-k_{a, R}}\right\|_{C^{0}\left[-R x_{a}^{*}, R x_{a}^{*}\right]} .
$$

2. We also have

$$
\begin{aligned}
& \sum_{i=1}^{2}\left\|\frac{\partial}{\partial x_{i}}\left(f_{a, R}-I d\right)\right\|_{C^{0}\left(\bar{\Omega}_{a, R}\right)} \\
\leq & \left\|\frac{k_{a, R}-g_{\delta}}{3 R-k_{a, R}}\right\|_{C^{0}\left[-R x_{a}^{*}, R x_{a}^{*}\right]}+4 R\left\|\frac{\partial}{\partial x} \frac{k_{a, R}-g_{\delta}}{3 R-k_{a, R}}\right\|_{C^{0}\left[-R x_{a}^{*}, R x_{a}^{*}\right]} .
\end{aligned}
$$


3. From the definition of the function $f_{a, R}$ in (3.5) we get

$$
\begin{aligned}
& \sum_{i, j=1}^{2}\left\|\frac{\partial^{2}}{\partial x_{i} x_{j}} f_{a, R}\right\|_{C^{0}\left(\bar{\Omega}_{a, R}\right)}+4 R\left\|\frac{\partial^{2}}{\partial x^{2}} \frac{k_{a, R}-g_{\delta}}{3 R-k_{a, R}}\right\|_{C^{0}\left[-R x_{a}^{*}, R x_{a}^{*}\right]} . \\
\leq & \left\|\frac{\partial}{\partial x} \frac{k_{a, R}-g_{\delta}}{3 R-k_{a, R}}\right\|_{C^{0}\left[-R x_{a}^{*}, R x_{a}^{*}\right]}+
\end{aligned}
$$

4. One finds

$$
\begin{aligned}
& \sum_{i, j=1}^{2}\left[\frac{\partial^{2}}{\partial x_{i} x_{j}} f_{a, R}\right]_{C^{\gamma}\left(\bar{\Omega}_{a, R}\right)} \\
= & {\left[(x, y) \mapsto(y-3 R) \frac{\partial^{2}}{\partial x^{2}} \frac{k_{a, R}(x)-g_{\delta}(x)}{3 R-k_{a, R}(x)}\right]_{C^{\gamma}\left(\bar{\Omega}_{a, R}\right)}+} \\
& +\left[\frac{\partial}{\partial x} \frac{k_{a, R}-g_{\delta}}{3 R-k_{a, R}}\right]_{C^{\gamma}\left[-R x_{a}^{*}, R x_{a}^{*}\right]}=\ldots
\end{aligned}
$$

Since it holds that

$$
[(x, y) \mapsto f(x) g(y)]_{C^{\alpha}[a, b]^{2}} \leq\|f\|_{C^{0}[a, b]}[g]_{C^{\alpha}[a, b]}+\|g\|_{C^{0}[a, b]}[f]_{C^{\alpha}[a, b]}
$$

one gets from (C.2) that

$$
\begin{aligned}
& \cdots \leq 3 R^{1-\gamma}\left\|\frac{\partial^{2}}{\partial x^{2}} \frac{k_{a, R}-g_{\delta}}{3 R-k_{a, R}}\right\|_{C^{0}\left[-R x_{a}^{*}, R x_{a}^{*}\right]}+ \\
& +4 R\left[\frac{\partial^{2}}{\partial x^{2}} \frac{k_{a, R}-g_{\delta}}{3 R-k_{a, R}}\right]_{C^{\gamma}\left[-R x_{a}^{*}, R x_{a}^{*}\right]}^{+2 R^{1-\gamma}}\left\|\frac{\partial^{2}}{\partial x^{2}} \frac{k_{a, R}-g_{\delta}}{3 R-k_{a, R}}\right\|_{C^{0}\left[-R x_{a}^{*}, R x_{a}^{*}\right]},
\end{aligned}
$$

and the claim follows.

\section{C.1.2 Proof of Proposition 3.11}

We divide the rather technical proof of Proposition 3.11 in several lemmas. Using the result of Proposition 3.10, to bound $\left\|f_{a, R}^{R}-I d\right\|_{C^{2, \gamma}\left(\bar{\Omega}_{a, 1}\right)}$ it is sufficient to get the estimates of the terms in the right hand side of (3.10) separately. We will do so in the next lemmas.

In the following $C_{i}=C_{i}(M)>0$, for $i=1, \ldots, 9$. The constants $N_{i}$, $i=1,2$ are defined in (3.7) and (3.8). 
Lemma C.2 For $k_{a, R}$ and $g_{\delta}$ respectively as in (2.3) and (3.4) it holds that

$$
\left\|\frac{k_{a, R}-g_{\delta}}{3 R-k_{a, R}}\right\|_{C^{0}\left[-R x_{a}^{*}, R x_{a}^{*}\right]} \leq C_{1} \delta R .
$$

Proof: By the definition of $g_{\delta}$ in (3.4), and (3.9) one has

$$
\begin{aligned}
& \left\|k_{a, R}-g_{\delta}\right\|_{C^{0}\left[-R x_{a}^{*}, R x_{a}^{*}\right]} \leq\left\|k_{a, R}-g\right\|_{C^{0}[-\delta, \delta]}+ \\
+ & \sum_{\sigma= \pm}\left\|\left.\varphi_{a, R}(\cdot) \sum_{i=0}^{2} \frac{1}{i !}\left(g-k_{a, R}\right)^{(i)}\right|_{\sigma \delta}(\cdot-\sigma \delta)^{i}\right\|_{C^{0}\left[\sigma \delta, \sigma R x_{a}^{*}\right]}+ \\
+ & \sum_{\sigma= \pm}\left\|\left.\psi_{a, \delta}(\cdot) \sum_{i=3}^{4} \frac{1}{i !}\left(g-k_{a, R}\right)^{(i)}\right|_{\sigma \delta}(\cdot-\sigma \delta)^{i}\right\|_{C^{0}[\sigma \delta, \sigma 2 \delta]} \\
\leq & N_{1} \delta^{3}+2 \sum_{i=0}^{2} \frac{1}{i !} N_{1} \delta^{3-i} R^{i}+\frac{2}{3 !} N_{1} \delta^{3}+\frac{2}{4 !} N_{2} \delta^{4} \leq C_{1} \delta R^{2} .
\end{aligned}
$$

The claim follows since $\left|k_{a, R}\right| \leq 2 R$.

Lemma C.3 Let $k_{a, R}$ and $g_{\delta}$ be given respectively as in (2.3) and (3.4). Then it holds

$$
\left\|\frac{\partial}{\partial x} \frac{k_{a, R}-g_{\delta}}{3 R-k_{a, R}}\right\|_{C^{0}\left[-R x_{a}^{*}, R x_{a}^{*}\right]} \leq C_{2} \delta .
$$

Proof: Using Lemma C.2 and (2.5) one finds directly

$$
\begin{aligned}
& \left\|\frac{\partial}{\partial x} \frac{k_{a, R}-g_{\delta}}{3 R-k_{a, R}}\right\|_{C^{0}\left[-R x_{a}^{*}, R x_{a}^{*}\right]} \leq \\
\leq & \frac{1}{R}\left\|\frac{\partial}{\partial x}\left(k_{a, R}-g_{\delta}\right)\right\|_{C^{0}\left[-R x_{a}^{*}, R x_{a}^{*}\right]}+C_{1} \delta b_{1} \leq \ldots
\end{aligned}
$$

By the definition of $g_{\delta}$ and the choice of the cut-off functions $\varphi_{a, R}$ and $\psi_{a, \delta}$ we get

$$
\begin{aligned}
\ldots & \leq \frac{N_{1}}{R} \delta^{2}+\frac{2}{R} \sum_{i=1}^{2} \frac{1}{(i-1) !} N_{1} \delta^{3-i} R^{i-1}+\frac{2}{R} \frac{D_{1,0}}{R} \sum_{i=0}^{2} \frac{1}{i !} N_{1} \delta^{3-i} R^{i}+ \\
+ & \frac{1}{2 !} \frac{2}{R} N_{1} \delta^{2}+\frac{1}{3 !} \frac{2}{R} \frac{D_{1,0}^{\prime}}{\delta} N_{1} \delta^{3}+\frac{1}{3 !} \frac{2}{R} N_{2} \delta^{3}+\frac{1}{4 !} \frac{2}{R} \frac{D_{1,0}^{\prime}}{\delta} N_{2} \delta^{4}+C_{1} b_{1} \delta \\
& \leq C_{2} \delta .
\end{aligned}
$$

Here we used (3.9) and that $\delta<R$ and $\delta<1$. 
Lemma C.4 For $k_{a, R}$ and $g_{\delta}$ respectively as in (2.3) and (3.4) it holds that

$$
\left\|\frac{\partial^{2}}{\partial x^{2}} \frac{k_{a, R}-g_{\delta}}{3 R-k_{a, R}}\right\|_{C^{0}\left[-R x_{a}^{*}, R x_{a}^{*}\right]} \leq C_{3} \frac{\delta}{R} .
$$

Proof: Since $\left(\frac{\alpha}{\beta}\right)^{\prime \prime}=\frac{1}{\beta} \alpha^{\prime \prime}-2 \frac{\beta^{\prime}}{\beta}\left(\frac{\alpha}{\beta}\right)^{\prime}-\frac{\beta^{\prime \prime}}{\beta} \frac{\alpha}{\beta}$, using Lemmas C.2 and C.3 and (2.5) one finds

$$
\begin{aligned}
\left\|\frac{\partial^{2}}{\partial x^{2}} \frac{k_{a, R}-g_{\delta}}{3 R-k_{a, R}}\right\|_{C^{0}\left[-R x_{a}^{*}, R x_{a}^{*}\right]} \leq & \frac{1}{R}\left\|\frac{\partial^{2}}{\partial x^{2}}\left(k_{a, R}-g_{\delta}\right)\right\|_{C^{0}\left[-R x_{a}^{*}, R x_{a}^{*}\right]} \\
& +\frac{2}{R} b_{1} C_{2} \delta+\frac{1}{R} \frac{b_{2}}{R} C_{1} \delta R \leq \ldots
\end{aligned}
$$

By the definition of $g_{\delta}$ in (3.4) one gets

$$
\begin{aligned}
& \cdots \leq \frac{1}{R} N_{1} \delta+\frac{2}{R} N_{1} \delta+\frac{4}{R} \frac{D_{1,0}}{R} \sum_{i=1}^{2} \frac{1}{(i-1) !} N_{1} \delta^{3-i} R^{i-1}+ \\
& \frac{2}{R} \frac{D_{2,0}}{R^{2}} \sum_{i=0}^{2} \frac{1}{i !} N_{1} \delta^{3-i} R^{i}+\frac{2}{R} N_{1} \delta+\frac{2}{R} \frac{1}{2} 2 \frac{D_{1,0}^{\prime}}{\delta} N_{1} \delta^{2}+\frac{2}{R} \frac{1}{3 !} N_{1} \delta^{3} \frac{D_{2,0}^{\prime}}{\delta^{2}}+ \\
& +\frac{2}{R} \frac{1}{2} N_{2} \delta^{2}+\frac{2}{R} \frac{2}{3 !} \frac{D_{1,0}^{\prime}}{\delta} N_{2} \delta^{3}+\frac{2}{R} \frac{1}{4 !} \frac{D_{2,0}^{\prime}}{\delta^{2}} N_{2} \delta^{4}+\frac{1}{R}\left(2 b_{1} C_{2}+b_{2} C_{1}\right) \delta \\
& \quad \leq C_{3} \frac{\delta}{R} .
\end{aligned}
$$

The constant $C_{3}$ depends on $\Omega$ through $N_{1}$ and $N_{2}$.

Remark C.5 Notice that the proof also implies that

$$
\left\|\frac{\partial^{2}}{\partial x^{2}}\left(k_{a, R}-g_{\delta}\right)\right\|_{C^{0}\left[-R x_{a}^{*}, R x_{a}^{*}\right]} \leq C_{4} \delta .
$$

Lemma C.6 For $k_{a, R}$ and $g_{\delta}$ respectively as in (2.3) and (3.4) it holds that

$$
\left[\frac{\partial^{2}}{\partial x^{2}}\left(k_{a, R}-g_{\delta}\right)\right]_{C \gamma\left[-R x_{a}^{*}, R x_{a}^{*}\right]} \leq C_{5} \delta^{1-\gamma}
$$


Proof: Writing explicitly the function $g_{\delta}$ yields

$$
\begin{aligned}
& {\left[\frac{\partial^{2}}{\partial x^{2}}\left(k_{a, R}-g_{\delta}\right)\right]_{C^{\gamma}\left[-R x_{a}^{*}, R x_{a}^{*}\right]} \leq 2 N_{1} \delta^{1-\gamma}+} \\
& +2\left[\frac{\partial^{2}}{\partial x^{2}}\left(\left.\varphi_{a, R}(\cdot) \sum_{i=0}^{2} \frac{1}{i !}\left(g-k_{a, R}\right)^{(i)}\right|_{\delta}(\cdot-\delta)^{i}\right)\right]_{C^{\gamma}\left[\delta, R x_{a}^{*}\right]} \\
& +2\left[\frac{\partial^{2}}{\partial x^{2}}\left(\left.\psi_{a, \delta}(\cdot) \sum_{i=3}^{4} \frac{1}{i !}\left(g-k_{a, R}\right)^{(i)}\right|_{\delta}(\cdot-\delta)^{i}\right)\right]_{C^{\gamma}[\delta, 2 \delta]} .
\end{aligned}
$$

It is convenient to study separately the terms on the right-hand side of (C.3). In the following $\tilde{C}_{i}=\tilde{C}_{i}(M)>0, i=1,2$.

1. By (3.9) one has

$$
\begin{aligned}
& {\left[\frac{\partial^{2}}{\partial x^{2}}\left(\left.\varphi_{a, R}(\cdot) \sum_{i=0}^{2} \frac{1}{i !}\left(g-k_{a, R}\right)^{(i)}\right|_{\delta}(\cdot-\delta)^{i}\right)\right]_{C^{\gamma}\left[\delta, R x_{a}^{*}\right]} } \\
& \leq \sum_{i=0}^{2} \frac{1}{i !} N_{1} \delta^{3-i}\left[(\cdot-\delta)^{i} \frac{\partial^{2}}{\partial x^{2}} \varphi_{a, R}\right]_{C^{\gamma}\left[\delta, R x_{a}^{*}\right]} \\
+ & 2 \sum_{i=1}^{2} \frac{1}{(i-1) !} N_{1} \delta^{3-i}\left[(\cdot-\delta)^{i-1} \frac{\partial}{\partial x} \varphi_{a, R}\right]_{C^{\gamma}\left[\delta, R x_{a}^{*}\right]}+N_{1} \delta\left[\varphi_{a, R}\right]_{C^{\gamma}\left[\delta, R x_{a}^{*}\right]} \leq
\end{aligned}
$$

Via the definition of the cut-off function $\varphi_{a, R}$ we get

$$
\begin{aligned}
& \leq \sum_{i=1}^{2} \frac{1}{(i-1) !} N_{1} \delta^{3-i} R^{i-1} R^{1-\gamma} \frac{D_{2,0}}{R^{2}}+ \\
& +\sum_{i=0}^{2} \frac{1}{i !} N_{1} \delta^{3-i} \frac{D_{2, \gamma}}{R^{2+\gamma}} R^{i}+2 N_{1} \delta R^{1-\gamma} \frac{D_{1,0}}{R} \\
& +2 \sum_{i=1}^{2} \frac{1}{(i-1) !} N_{1} \delta^{3-i} \frac{D_{1, \gamma}}{R^{1+\gamma}} R^{i-1}+N_{1} \delta \frac{D_{0, \gamma}}{R^{\gamma}} \leq \tilde{C}_{1} \delta^{1-\gamma}
\end{aligned}
$$




\section{Since}

$$
\begin{aligned}
& {\left[\frac{\partial^{2}}{\partial x^{2}}\left(\left.\psi_{a, \delta}(\cdot) \sum_{i=3}^{4} \frac{1}{i !}\left(g-k_{a, R}\right)^{(i)}\right|_{\delta}(\cdot-\delta)^{i}\right)\right]_{C^{\gamma}[\delta, 2 \delta]} } \\
\leq & \left.\sum_{i=3}^{4} \frac{1}{i !}\left(g-k_{a, R}\right)^{(i)}\right|_{\delta}\left[(\cdot-\delta)^{i} \frac{\partial^{2}}{\partial x^{2}} \psi_{a, \delta}(\cdot)\right]_{C^{\gamma}[\delta, 2 \delta]} \\
& +\left.\sum_{i=3}^{4} \frac{2}{(i-1) !}\left(g-k_{a, R}\right)^{(i)}\right|_{\delta}\left[(\cdot-\delta)^{i-1} \frac{\partial}{\partial x} \psi_{a, \delta}(\cdot)\right]_{C^{\gamma}[\delta, 2 \delta]} \\
& +\left.\sum_{i=3}^{4} \frac{1}{(i-2) !}\left(g-k_{a, R}\right)^{(i)}\right|_{\delta}\left[(\cdot-\delta)^{i-2} \psi_{a, \delta}(\cdot)\right]_{C^{\gamma}[\delta, 2 \delta]} \leq
\end{aligned}
$$

from (3.9) and the choice of $\psi_{a, \delta}$ one obtains

$$
\begin{aligned}
\leq & \left.\sum_{i=3}^{4} \frac{1}{i !}\left(g-k_{a, R}\right)^{(i)}\right|_{\delta}\left(\delta^{i} \frac{D_{2, \gamma}^{\prime}}{\delta^{2+\gamma}}+i \delta^{i-1} \delta^{1-\gamma} \frac{D_{2,0}^{\prime}}{\delta^{2}}\right) \\
& +\left.\sum_{i=3}^{4} \frac{2}{(i-1) !}\left(g-k_{a, R}\right)^{(i)}\right|_{\delta}\left(\delta^{i-1} \frac{D_{1, \gamma}^{\prime}}{\delta^{1+\gamma}}+(i-1) \delta^{i-2} \delta^{1-\gamma} \frac{D_{1,0}^{\prime}}{\delta}\right) \\
& +\left.\sum_{i=3}^{4} \frac{1}{(i-2) !}\left(g-k_{a, R}\right)^{(i)}\right|_{\delta}\left(\delta^{i-2} \frac{D_{0, \gamma}^{\prime}}{\delta^{\gamma}}+(i-2) \delta^{i-3} \delta^{1-\gamma}\right) \leq \tilde{C}_{2} \delta^{1-\gamma} .
\end{aligned}
$$

The claim follows.

Lemma C.7 Let $k_{a, R}$ and $g_{\delta}$ be given respectively in (2.3) and (3.4). Then it holds

$$
\left[\frac{\partial^{2}}{\partial x^{2}} \frac{k_{a, R}-g_{\delta}}{3 R-k_{a, R}}\right]_{C^{\gamma}\left[-R x_{a}^{*}, R x_{a}^{*}\right]} \leq \frac{C_{9}}{R} \delta^{1-\gamma}
$$


Proof: We have, writing $[\cdot]_{C^{\gamma}}:=[\cdot]_{C^{\gamma}\left[-R x_{a}^{*}, R x_{a}^{*}\right]}$, that

$$
\begin{aligned}
& {\left[\frac{\partial^{2}}{\partial x^{2}} \frac{k_{a, R}-g_{\delta}}{3 R-k_{a, R}}\right]_{C^{\gamma}} \leq\left[\frac{1}{3 R-k_{a, R}} \frac{\partial^{2}}{\partial x^{2}}\left(k_{a, R}-g_{\delta}\right)\right]_{C^{\gamma}}} \\
& +2\left[\frac{1}{3 R-k_{a, R}} \frac{\partial}{\partial x} k_{a, R} \frac{\partial}{\partial x} \frac{k_{a, R}-g_{\delta}}{3 R-k_{a, R}}\right]_{C^{\gamma}}+ \\
& +\left[\frac{k_{a, R}-g_{\delta}}{\left(3 R-k_{a, R}\right)^{2}} \frac{\partial^{2}}{\partial x^{2}} k_{a, R}\right]_{C^{\gamma}} .
\end{aligned}
$$

We study the terms in the right-hand side of (C.4) separately.

1. From (2.5), Remark C.5 and Lemma C.6 it follows that

$$
\begin{aligned}
& {\left[\frac{1}{3 R-k_{a, R}} \frac{\partial^{2}}{\partial x^{2}}\left(k_{a, R}-g_{\delta}\right)\right]_{C^{\gamma}} \leq \frac{1}{R^{2}} b_{1} 2 R^{1-\gamma} C_{4} \delta+\frac{C_{5}}{R} \delta^{1-\gamma}} \\
& \leq \frac{C_{6}}{R} \delta^{1-\gamma}
\end{aligned}
$$

2. Using (2.5) and Lemmas C.3 and C.4 one obtains

$$
\begin{aligned}
& {\left[\frac{1}{3 R-k_{a, R}} \frac{\partial}{\partial x} k_{a, R} \frac{\partial}{\partial x} \frac{k_{a, R}-g_{\delta}}{3 R-k_{a, R}}\right]_{C \gamma} } \\
\leq & b_{1} C_{2} \delta\left[\frac{1}{3 R-k_{a, R}}\right]_{C^{\gamma}}+\frac{1}{R} C_{2} \delta\left[\frac{\partial}{\partial x} k_{a, R}\right]_{C^{\gamma}} \\
& +b_{1} \frac{1}{R}\left[\frac{\partial}{\partial x} \frac{k_{a, R}-g_{\delta}}{3 R-k_{a, R}}\right]_{C^{\gamma}} \\
\leq & \frac{1}{R^{2}} b_{1} 2 R^{1-\gamma} C_{2} b_{1} \delta+\frac{b_{2}}{R} 2 R^{1-\gamma} \frac{C_{2}}{R} \delta+C_{3} \frac{\delta}{R} 2 R^{1-\gamma} \frac{b_{1}}{R} \leq \frac{C_{7}}{R} \delta^{1-\gamma} .
\end{aligned}
$$

3. Since

$$
\begin{aligned}
& {\left[\frac{k_{a, R}-g_{\delta}}{\left(3 R-k_{a, R}\right)^{2}} \frac{\partial^{2}}{\partial x^{2}} k_{a, R}\right]_{C^{\gamma}} } \\
\leq & {\left[\frac{k_{a, R}-g_{\delta}}{3 R-k_{a, R}}\right]_{C^{\gamma}} \frac{1}{R} \frac{b_{2}}{R}+\left[\frac{1}{3 R-k_{a, R}}\right]_{C^{\gamma}} \frac{b_{2}}{R} C_{1} \delta R+} \\
& +\left[\frac{\partial^{2}}{\partial x^{2}} k_{a, R}\right]_{C^{\gamma}} \frac{1}{R} C_{1} \delta R \leq \ldots,
\end{aligned}
$$


applying (2.5) and Lemmas C.2 and C.3 one finds

$\cdots \leq C_{2} \delta 2 R^{1-\gamma} \frac{b_{2}}{R^{2}}+\frac{1}{R^{2}} 2 b_{1} R^{1-\gamma} b_{2} C_{1} \delta+\frac{b_{3}}{R^{2}} 2 R^{1-\gamma} C_{1} \delta \leq \frac{C_{8}}{R} \delta^{1-\gamma}$.

The claim follows directly from (C.4) using the results of the previous points 1,2 and 3 .

The proof of Proposition 3.11 follows from Lemmas C.2, C.3, C.4 and C.7.

\section{C.2 Bounded third and fourth derivative of the mapping}

\section{C.2.1 Proof of Proposition 3.12}

Proof of Proposition 3.12: Let $f_{a, R, 1}$ and $f_{a, R, 2}$ be respectively the first and the second component of $f_{a, R}$. From the definition of $f_{a, R}$ we find: $f_{a, R, 1}(x, y)=x$ and $f_{a, R, 2}(x, y)=\frac{3 R-g_{\delta}(x)}{3 R-k_{a, R}(x)}(y-3 R)+3 R$. Hence $\left\|f_{a, R, 1}\right\|_{C^{4, \alpha}\left(\bar{\Omega}_{a, 1}\right)} \leq x_{a}+1$ and Lemma C.1 yields

$$
\begin{aligned}
\left\|f_{a, R, 2}^{R}\right\|_{C^{4, \alpha}\left(\bar{\Omega}_{a, 1}\right)} \leq & \sum_{\substack{|\beta|=0, \beta \in \mathbb{N}^{2}}}^{4} R^{|\beta|-1}\left\|D^{\beta} f_{a, R, 2}\right\|_{C^{0}\left(\bar{\Omega}_{a, R}\right)} \\
& +R^{3+\alpha} \sum_{\substack{|\beta|=4, \beta \in \mathbb{N}^{2}}}\left[D^{\beta} f_{a, R, 2}\right]_{C^{\alpha}\left(\bar{\Omega}_{a, R}\right)} \leq \ldots
\end{aligned}
$$

By observing that

$$
\frac{1}{R}\left\|f_{a, R, 2}\right\|_{C^{0}\left(\bar{\Omega}_{a, R}\right)} \leq 3+4\left\|\frac{3 R-g_{\delta}}{3 R-k_{a, R}}\right\|_{C^{0}\left(-R x_{a}^{*}, R x_{a}^{*}\right)},
$$

using that $f_{a, R, 2}$ is linear in $y$ one finds

$$
\begin{aligned}
\ldots \leq & 3+5 \sum_{i=0}^{3} R^{i}\left\|\frac{\partial^{i}}{\partial x^{i}} \frac{3 R-g_{\delta}}{3 R-k_{a, R}}\right\|_{C^{0}\left(-R x_{a}^{*}, R x_{a}^{*}\right)}+ \\
& +6 R^{4}\left\|\frac{\partial^{4}}{\partial x^{4}} \frac{3 R-g_{\delta}}{3 R-k_{a, R}}\right\|_{C^{0}\left(-R x_{a}^{*}, R x_{a}^{*}\right)} \\
& +R^{3+\alpha}\left[(x, y) \mapsto(y-3 R) \frac{\partial^{4}}{\partial x^{4}} \frac{3 R-g_{\delta}}{3 R-k_{a, R}}\right]_{C^{\alpha}\left(\bar{\Omega}_{a, R}\right)} .
\end{aligned}
$$


The claim follows from Lemmas C.2, C.3 and C.4 since $\frac{3 R-g_{\delta}}{3 R-k_{a, R}}=$ $1+\frac{k_{a, R}-g_{\delta}}{3 R-k_{a, R}}$.

\section{C.2.2 Proof of Proposition 3.13}

We also divide the technical proof of Proposition 3.13 in several lemmas.

In the following $C_{i}=C_{i}(M)>0$, for $i=12, \ldots, 18$. The constants $N_{i}, i=1,2$ are defined in (3.7) and (3.8).

Lemma C.8 For $k_{a, R}$ and $g_{\delta}$ respectively as in (2.3) and (3.4) it holds that

$$
\left\|\frac{\partial^{3}}{\partial x^{3}}\left(k_{a, R}-g_{\delta}\right)\right\|_{C^{0}\left[-R x_{a}^{*}, R x_{a}^{*}\right]} \leq C_{12} .
$$

Proof: By the definition of $g_{\delta}$ we have

$$
\begin{aligned}
& \left\|\frac{\partial^{3}}{\partial x^{3}}\left(k_{a, R}-g_{\delta}\right)\right\|_{C^{0}\left[-R x_{a}^{*}, R x_{a}^{*}\right]} \leq\left\|\frac{\partial^{3}}{\partial x^{3}}\left(k_{a, R}-g\right)\right\|_{C^{0}[-\delta, \delta]}+ \\
& +2\left\|\frac{\partial^{3}}{\partial x^{3}}\left(\left.\varphi_{a, R}(\cdot) \sum_{i=0}^{2} \frac{1}{i !}\left(g-k_{a, R}\right)^{(i)}\right|_{\delta}(\cdot-\delta)^{i}\right)\right\|_{C^{0}\left[\delta, R x_{a}^{*}\right]} \\
& +2\left\|\frac{\partial^{3}}{\partial x^{3}}\left(\left.\psi_{a, \delta}(\cdot) \sum_{i=3}^{4} \frac{1}{i !}\left(g-k_{a, R}\right)^{(i)}\right|_{\delta}(\cdot-\delta)^{i}\right)\right\|_{C^{0}[\delta, 2 \delta]} \cdot
\end{aligned}
$$

It is convenient to study the terms on the right-hand side of (C.5) separately. In the following $\bar{C}_{i}=\bar{C}_{i}(M)>0$ for $i=1,2$.

1. It follows directly from (3.9) that $\left\|\frac{\partial^{3}}{\partial x^{3}}\left(k_{a, R}-g\right)\right\|_{C^{0}[-\delta, \delta]} \leq N_{1}$.

2. Via (3.9) and the definition of the cut-off function $\varphi_{a, R}$ we get

$$
\begin{aligned}
& \left\|\frac{\partial^{3}}{\partial x^{3}}\left(\left.\varphi_{a, R}(\cdot) \sum_{i=0}^{2} \frac{1}{i !}\left(g-k_{a, R}\right)^{(i)}\right|_{\delta}(\cdot-\delta)^{i}\right)\right\|_{C^{0}\left[\delta, R x_{a}^{*}\right]} \\
\leq & \sum_{i=0}^{2} \frac{1}{i !} N_{1} \delta^{3-i} \frac{D_{3,0}}{R^{3}} R^{i}+3 \sum_{i=1}^{2} \frac{1}{(i-1) !} N_{1} \delta^{3-i} \frac{D_{2,0}}{R^{2}} R^{i-1}+ \\
& +3 N_{1} \delta \frac{D_{1,0}}{R} \leq \frac{\bar{C}_{1}}{R} \delta<\bar{C}_{1} .
\end{aligned}
$$


3. One finds

$$
\begin{gathered}
\left\|\frac{\partial^{3}}{\partial x^{3}}\left(\left.\psi_{a, \delta}(\cdot) \sum_{i=3}^{4} \frac{1}{i !}\left(g-k_{a, R}\right)^{(i)}\right|_{\delta}(\cdot-\delta)^{i}\right)\right\|_{C^{0}[\delta, 2 \delta]} \leq \\
\leq\left.\sum_{i=3}^{4} \frac{1}{i !}\left(g-k_{a, R}\right)^{(i)}\right|_{\delta} \frac{D_{3,0}^{\prime}}{\delta^{3}} \delta^{i}+\left.3 \sum_{i=3}^{4} \frac{1}{(i-1) !}\left(g-k_{a, R}\right)^{(i)}\right|_{\delta} \frac{D_{2,0}^{\prime}}{\delta^{2}} \delta^{i-1}+ \\
+\left.3 \sum_{i=3}^{4} \frac{1}{(i-2) !}\left(g-k_{a, R}\right)^{(i)}\right|_{\delta} \frac{D_{1,0}^{\prime}}{\delta} \delta^{i-2}+\left.\sum_{i=3}^{4}\left(g-k_{a, R}\right)^{(i)}\right|_{\delta} \delta^{i-3} \leq \bar{C}_{2} .
\end{gathered}
$$

The claim follows.

Lemma C.9 For $k_{a, R}$ and $g_{\delta}$ respectively as in (2.3) and (3.4) it holds that

$$
\left\|\frac{\partial^{3}}{\partial x^{3}} \frac{k_{a, R}-g_{\delta}}{3 R-k_{a, R}}\right\|_{C^{0}\left(-R x_{a}^{*}, R x_{a}^{*}\right)} \leq \frac{C_{13}}{R} .
$$

Proof: Since

$$
\left(\frac{\alpha}{\beta}\right)^{\prime \prime \prime}=\frac{\alpha^{\prime \prime \prime}}{\beta}-3 \frac{\beta^{\prime}}{\beta}\left(\frac{\alpha}{\beta}\right)^{\prime \prime}-3 \frac{\beta^{\prime \prime}}{\beta}\left(\frac{\alpha}{\beta}\right)^{\prime}-\frac{\beta^{\prime \prime \prime}}{\beta} \frac{\alpha}{\beta},
$$

using Lemma C.8, (2.5) and Lemmas C.4, C.3, C.2 we get

$$
\begin{aligned}
& \left\|\frac{\partial^{3}}{\partial x^{3}} \frac{k_{a, R}-g_{\delta}}{3 R-k_{a, R}}\right\|_{C^{0}\left(-R x_{a}^{*}, R x_{a}^{*}\right)} \\
& \leq \frac{1}{R} C_{12}+3 \frac{b_{1}}{R} \frac{C_{3} \delta}{R}+3 \frac{b_{2}}{R^{2}} C_{2} \delta+\frac{b_{3}}{R^{3}} C_{1} \delta R \leq \frac{C_{13}}{R} .
\end{aligned}
$$

Lemma C.10 For $k_{a, R}$ and $g_{\delta}$ respectively as in (2.3) and (3.4) it holds that

$$
\left\|\frac{\partial^{4}}{\partial x^{4}}\left(k_{a, R}-g_{\delta}\right)\right\|_{C^{0}\left[-R x_{a}^{*}, R x_{a}^{*}\right]} \leq \frac{C_{14}}{\delta} .
$$


Proof: From the definition of $g_{\delta}$ it follows

$$
\begin{aligned}
& \left\|\frac{\partial^{4}}{\partial x^{4}}\left(k_{a, R}-g_{\delta}\right)\right\|_{C^{0}\left[-R x_{a}^{*}, R x_{a}^{*}\right]} \leq\left\|\frac{\partial^{4}}{\partial x^{4}}\left(k_{a, R}-g\right)\right\|_{C^{0}[-\delta, \delta]}+ \\
& +2\left\|\frac{\partial^{4}}{\partial x^{4}}\left(\left.\varphi_{a, R}(\cdot) \sum_{i=0}^{2} \frac{1}{i !}\left(g-k_{a, R}\right)^{(i)}\right|_{\delta}(\cdot-\delta)^{i}\right)\right\|_{C^{0}\left[\delta, R x_{a}^{*}\right]}+ \\
& +2\left\|\frac{\partial^{4}}{\partial x^{4}}\left(\left.\psi_{a, \delta}(\cdot) \sum_{i=3}^{4} \frac{1}{i !}\left(g-k_{a, R}\right)^{(i)}\right|_{\delta}(\cdot-\delta)^{i}\right)\right\|_{C^{0}[\delta, 2 \delta]} \cdot
\end{aligned}
$$

It is convenient to study the terms on the right-hand side of (C.6) separately. Here $\tilde{C}_{i}=\tilde{C}_{i}(M)>0$ for $i=1,2$.

1. From (3.9) it follows directly that $\left\|\frac{\partial^{4}}{\partial x^{4}}\left(k_{a, R}-g\right)\right\|_{C^{0}[-\delta, \delta]} \leq N_{2}$.

2. By (3.9) and the definition of the cut-off function $\varphi_{a, R}$ we get that

$$
\begin{aligned}
& \left\|\frac{\partial^{4}}{\partial x^{4}}\left(\left.\varphi_{a, R}(\cdot) \sum_{i=0}^{2} \frac{1}{i !}\left(g-k_{a, R}\right)^{(i)}\right|_{\delta}(\cdot-\delta)^{i}\right)\right\|_{C^{0}\left[\delta, R x_{a}^{*}\right]} \\
\leq & \sum_{i=0}^{2} \frac{1}{i !} N_{1} \delta^{3-i} \frac{D_{4,0}}{R^{4}} R^{i}+4 \sum_{i=1}^{2} \frac{1}{(i-1) !} N_{1} \delta^{3-i} \frac{D_{3,0}}{R^{3}} R^{i-1}+6 N_{1} \delta \frac{D_{2,0}}{R^{2}} \\
\leq & \frac{\tilde{C}_{1}}{R^{2}} \delta<\frac{\tilde{C}_{1}}{R} .
\end{aligned}
$$

3. From (3.9) and the choice of $\psi_{a, \delta}$ one obtains

$$
\begin{aligned}
& \left\|\frac{\partial^{4}}{\partial x^{4}}\left(\left.\psi_{a, \delta}(\cdot) \sum_{i=3}^{4} \frac{1}{i !}\left(g-k_{a, R}\right)^{(i)}\right|_{\delta}(\cdot-\delta)^{i}\right)\right\|_{C^{0}[\delta, 2 \delta]} \\
\leq & \left.\sum_{i=3}^{4} \frac{1}{i !}\left(g-k_{a, R}\right)^{(i)}\right|_{\delta} \frac{D_{4,0}^{\prime}}{\delta^{4}} \delta^{i}+\left.4 \sum_{i=3}^{4} \frac{1}{(i-1) !}\left(g-k_{a, R}\right)^{(i)}\right|_{\delta} \frac{D_{3,0}^{\prime}}{\delta^{3}} \delta^{i-1} \\
& +\left.6 \sum_{i=3}^{4} \frac{1}{(i-2) !}\left(g-k_{a, R}\right)^{(i)}\right|_{\delta} \frac{D_{2,0}^{\prime}}{\delta^{2}} \delta^{i-2}+\left.4 \sum_{i=3}^{4}\left(g-k_{a, R}\right)^{(i)}\right|_{\delta} \frac{D_{1,0}^{\prime}}{\delta} \delta^{i-3} \\
& +\left.\left(g-k_{a, R}\right)^{(4)}\right|_{\delta} \leq \frac{\tilde{C}_{2}}{\delta} .
\end{aligned}
$$

The claim follows. 
Lemma C.11 Let $k_{a, R}$ and $g_{\delta}$ be given respectively in (2.3) and (3.4). Then it holds that

$$
\left\|\frac{\partial^{4}}{\partial x^{4}} \frac{k_{a, R}-g_{\delta}}{3 R-k_{a, R}}\right\|_{C^{0}\left(-R x_{a}^{*}, R x_{a}^{*}\right)} \leq \frac{C_{15}}{\delta R} .
$$

Proof: From

$$
\left(\frac{\alpha}{\beta}\right)^{(i v)}=\frac{\alpha^{(i v)}}{\beta}-4 \frac{\beta^{\prime}}{\beta}\left(\frac{\alpha}{\beta}\right)^{\prime \prime \prime}-6 \frac{\beta^{\prime \prime}}{\beta}\left(\frac{\alpha}{\beta}\right)^{\prime \prime}-4 \frac{\beta^{\prime \prime \prime}}{\beta}\left(\frac{\alpha}{\beta}\right)^{\prime}-\frac{\beta^{(i v)}}{\beta} \frac{\alpha}{\beta},
$$

using Lemma C.10, (2.5) and Lemmas C.9, C.4, C.3, C.2 we get

$$
\begin{aligned}
\left\|\frac{\partial^{4}}{\partial x^{4}} \frac{k_{a, R}-g_{\delta}}{3 R-k_{a, R}}\right\|_{C^{0}\left(-R x_{a}^{*}, R x_{a}^{*}\right)} \leq & \frac{1}{R} \frac{C_{14}}{\delta}+4 \frac{b_{1}}{R} \frac{C_{13}}{R}+6 \frac{b_{2}}{R^{2}} C_{3} \frac{\delta}{R}+4 \frac{b_{3}}{R^{3}} C_{2} \delta \\
& +\frac{b_{4}}{R^{4}} C_{1} \delta R \leq \frac{C_{15}}{\delta R} .
\end{aligned}
$$

Lemma C.12 For $k_{a, R}$ and $g_{\delta}$ respectively as in (2.3) and (3.4) it holds that

$$
\left[\frac{\partial^{4}}{\partial x^{4}}\left(k_{a, R}-g_{\delta}\right)\right]_{C^{\alpha}\left[-R x_{a}^{*}, R x_{a}^{*}\right]} \leq \frac{C_{16}}{\delta^{1+\alpha}} .
$$

Proof: From the definition of $g_{\delta}$ one finds

$$
\begin{aligned}
& {\left[\frac{\partial^{4}}{\partial x^{4}}\left(k_{a, R}-g_{\delta}\right)\right]_{C^{\alpha}\left[-R x_{a}^{*}, R x_{a}^{*}\right]} \leq\left[\frac{\partial^{4}}{\partial x^{4}}\left(k_{a, R}-g\right)\right]_{C^{\alpha}[-\delta, \delta]}+} \\
& +2\left[\frac{\partial^{4}}{\partial x^{4}}\left(\left.\varphi_{a, R}(\cdot) \sum_{i=0}^{2} \frac{1}{i !}\left(g-k_{a, R}\right)^{(i)}\right|_{\delta}(\cdot-\delta)^{i}\right)\right]_{C^{\alpha}\left[\delta, R x_{a}^{*}\right]} \\
& +2\left[\frac{\partial^{4}}{\partial x^{4}}\left(\left.\psi_{a, \delta}(\cdot) \sum_{i=3}^{4} \frac{1}{i !}\left(g-k_{a, R}\right)^{(i)}\right|_{\delta}(\cdot-\delta)^{i}\right)\right]_{C^{\alpha}[\delta, 2 \delta]} .
\end{aligned}
$$

It is convenient to study the terms on the right-hand side of (C.8) separately. In the following $\tilde{C}_{i}=\tilde{C}_{i}(M)>0$ for $i=1,2,3$. 
1. Since $\Omega$ is a $C^{4, \alpha}$ domain with constant $M$ we have

$$
\left[\frac{\partial^{4}}{\partial x^{4}}\left(k_{a, R}-g\right)\right]_{C^{\alpha}[-\delta, \delta]} \leq\left[\frac{\partial^{4}}{\partial x^{4}} k_{a, R}\right]_{C^{\alpha}[-\delta, \delta]}+M \leq \tilde{C}_{1} .
$$

Notice that the constant $\tilde{C}_{1}$ depends only on $M$.

2. One has

$$
\begin{aligned}
& {\left[\frac{\partial^{4}}{\partial x^{4}}\left(\left.\varphi_{a, R}(\cdot) \sum_{i=0}^{2} \frac{1}{i !}\left(g-k_{a, R}\right)^{(i)}\right|_{\delta}(\cdot-\delta)^{i}\right)\right]_{C^{\alpha}\left[\delta, R x_{a}^{*}\right]} } \\
\leq & \left.\sum_{i=0}^{2} \frac{1}{i !}\left(g-k_{a, R}\right)^{(i)}\right|_{\delta}\left[(\cdot-\delta)^{i} \frac{\partial^{4}}{\partial x^{4}} \varphi_{a, R}(\cdot)\right]_{C^{\alpha}\left[\delta, R x_{a}^{*}\right]} \\
& +\left.4 \sum_{i=1}^{2} \frac{1}{(i-1) !}\left(g-k_{a, R}\right)^{(i)}\right|_{\delta}\left[(\cdot-\delta)^{i-1} \frac{\partial^{3}}{\partial x^{3}} \varphi_{a, R}(\cdot)\right]_{C^{\alpha}\left[\delta, R x_{a}^{*}\right]} \\
& +\left.6\left(g-k_{a, R}\right)^{(2)}\right|_{\delta}\left[\frac{\partial^{2}}{\partial x^{2}} \varphi_{a, R}\right]_{C^{\alpha}\left[\delta, R x_{a}^{*}\right]} \leq \ldots
\end{aligned}
$$

Via (3.9) and the definition of the cut-off function $\varphi_{a, R}$ we get

$$
\begin{aligned}
\ldots \leq & \sum_{i=0}^{2} \frac{1}{i !} N_{1} \delta^{3-i} \frac{D_{4, \alpha}}{R^{4+\alpha}} R^{i}+\sum_{i=1}^{2} \frac{1}{(i-1) !} N_{1} \delta^{3-i} \frac{D_{4,0}}{R^{4}} R^{i-1} R^{1-\alpha} \\
& +4 \sum_{i=1}^{2} \frac{1}{(i-1) !} N_{1} \delta^{3-i} \frac{D_{3, \alpha}}{R^{3+\alpha}} R^{i-1}+4 N_{1} \delta \frac{D_{3,0}}{R^{3}} R^{1-\alpha}+6 N_{1} \delta \frac{D_{2, \alpha}}{R^{2+\alpha}} \\
\leq & \frac{\tilde{C}_{2}}{R^{2+\alpha}} \delta
\end{aligned}
$$

\section{Since}

$$
\begin{aligned}
& {\left[\frac{\partial^{4}}{\partial x^{4}}\left(\left.\psi_{a, \delta}(\cdot) \sum_{i=3}^{4} \frac{1}{i !}\left(g-k_{a, R}\right)^{(i)}\right|_{\delta}(\cdot-\delta)^{i}\right)\right]_{C^{\alpha}[\delta, 2 \delta]} } \\
\leq & \left.\sum_{i=3}^{4} \frac{1}{i !}\left(g-k_{a, R}\right)^{(i)}\right|_{\delta}\left[(\cdot-\delta)^{i} \frac{\partial^{4}}{\partial x^{4}} \psi_{a, \delta}(\cdot)\right]_{C^{\alpha}[\delta, 2 \delta]}
\end{aligned}
$$




$$
\begin{aligned}
& +\left.\sum_{i=3}^{4} \frac{4}{(i-1) !}\left(g-k_{a, R}\right)^{(i)}\right|_{\delta}\left[(\cdot-\delta)^{i-1} \frac{\partial^{3}}{\partial x^{3}} \psi_{a, \delta}(\cdot)\right]_{C^{\alpha}[\delta, 2 \delta]} \\
& +\left.\sum_{i=3}^{4} \frac{6}{(i-2) !}\left(g-k_{a, R}\right)^{(i)}\right|_{\delta}\left[(\cdot-\delta)^{i-2} \frac{\partial^{2}}{\partial x^{2}} \psi_{a, \delta}(\cdot)\right]_{C^{\alpha}[\delta, 2 \delta]} \\
& +\left.4 \sum_{i=3}^{4}\left(g-k_{a, R}\right)^{(i)}\right|_{\delta}\left[(\cdot-\delta)^{i-3} \frac{\partial}{\partial x} \psi_{a, \delta}(\cdot)\right]_{C^{\alpha}[\delta, 2 \delta]} \\
& +\left.\left(g-k_{a, R}\right)^{(4)}\right|_{\delta}\left[\psi_{a, \delta}\right]_{C^{\alpha}[\delta, 2 \delta]} \leq \ldots,
\end{aligned}
$$

from (3.9) and the choice of $\psi_{a, \delta}$ one obtains

$$
\begin{aligned}
\ldots \leq & \left.\sum_{i=3}^{4} \frac{1}{i !}\left(g-k_{a, R}\right)^{(i)}\right|_{\delta}\left(\delta^{i} \frac{D_{4, \alpha}^{\prime}}{\delta^{4+\alpha}}+i \delta^{i-1} \delta^{1-\alpha} \frac{D_{4,0}^{\prime}}{\delta^{4}}\right) \\
& +\left.\sum_{i=3}^{4} \frac{4}{(i-1) !}\left(g-k_{a, R}\right)^{(i)}\right|_{\delta}\left(\delta^{i-1} \frac{D_{3, \alpha}^{\prime}}{\delta^{3+\alpha}}+(i-1) \delta^{i-2} \delta^{1-\alpha} \frac{D_{3,0}^{\prime}}{\delta^{3}}\right)+ \\
& +\left.\sum_{i=3}^{4} \frac{6}{(i-2) !}\left(g-k_{a, R}\right)^{(i)}\right|_{\delta}\left(\delta^{i-2} \frac{D_{2, \alpha}^{\prime}}{\delta^{2+\alpha}}+(i-2) \delta^{i-3} \delta^{1-\alpha} \frac{D_{2,0}^{\prime}}{\delta^{2}}\right) \\
& +\left.4 \sum_{i=3}^{4}\left(g-k_{a, R}\right)^{(i)}\right|_{\delta}\left(\delta^{i-3} \frac{D_{1, \alpha}^{\prime}}{\delta^{1+\alpha}}+(i-3) \delta^{i-4} \delta^{1-\alpha} \frac{D_{1,0}^{\prime}}{\delta}\right) \\
& +\left.\left(g-k_{a, R}\right)^{(4)}\right|_{\delta} \frac{D_{0, \alpha}^{\prime}}{\delta^{\alpha}} \leq \frac{\tilde{C}_{3}}{\delta^{1+\alpha}} .
\end{aligned}
$$

The claim follows.

Lemma C.13 For $k_{a, R}$ and $g_{\delta}$ respectively as in (2.3) and (3.4) it holds that

$$
\left[\frac{\partial^{4}}{\partial x^{4}} \frac{k_{a, R}-g_{\delta}}{3 R-k_{a, R}}\right]_{C^{\alpha}\left[-R x_{a}^{*}, R x_{a}^{*}\right]} \leq \frac{C_{17}}{R \delta^{1+\alpha}} .
$$

Proof: By (C.7) through Lemma C.10, (2.5) and Lemmas C.12, C.9, C.4, C.3, C.2 one obtains for $C^{\alpha}=C^{\alpha}\left(-R x_{a}^{*}, R x_{a}^{*}\right)$

$$
\left[\frac{\partial^{4}}{\partial x^{4}} \frac{k_{a, R}-g_{\delta}}{3 R-k_{a, R}}\right]_{C^{\alpha}} \leq\left[\frac{1}{3 R-k_{a, R}}\right]_{C^{\alpha}} \frac{C_{14}}{\delta}+\frac{1}{R} \frac{C_{16}}{\delta^{1+\alpha}}+
$$




$$
\begin{aligned}
& +4\left[\frac{1}{3 R-k_{a, R}}\right]_{C^{\alpha}} b_{1} \frac{C_{13}}{R}+4\left[\frac{\partial}{\partial x} k_{a, R}\right]_{C^{\alpha}} \frac{1}{R} \frac{C_{13}}{R}+ \\
& +4\left[\frac{\partial^{3}}{\partial x^{3}} \frac{k_{a, R}-g_{\delta}}{3 R-k_{a, R}}\right]_{C^{\alpha}} \frac{b_{1}}{R}+6\left[\frac{1}{3 R-k_{a, R}}\right]_{C^{\alpha}} \frac{b_{2}}{R} C_{3} \frac{\delta}{R}+ \\
& +6\left[\frac{\partial^{2}}{\partial x^{2}} k_{a, R}\right]_{C^{\alpha}} \frac{1}{R} C_{3} \frac{\delta}{R}+6\left[\frac{\partial^{2}}{\partial x^{2}} \frac{k_{a, R}-g_{\delta}}{3 R-k_{a, R}}\right]_{C^{\alpha}} \frac{b_{2}}{R^{2}}+ \\
& +4\left[\frac{1}{3 R-k_{a, R}}\right]_{C^{\alpha}} \frac{b_{3}}{R^{2}} C_{2} \delta+4\left[\frac{\partial^{3}}{\partial x^{3}} k_{a, R}\right]_{C^{\alpha}} \frac{1}{R} C_{2} \delta+ \\
& +4\left[\frac{\partial}{\partial x} \frac{k_{a, R}-g_{\delta}}{3 R-k_{a, R}}\right]_{C^{\alpha}} \frac{b_{3}}{R^{3}}+\left[\frac{b_{4}}{3 R-k_{a, R}}\right]_{C^{\alpha}} \frac{b^{3}}{R^{3}}+ \\
& +\left[\frac{\partial^{4}}{\partial x^{4}} k_{a, R}\right]_{C^{\alpha}} \frac{1}{R} C_{1} \delta R+\left[\frac{k_{a, R}-g_{\delta}}{3 R-k_{a, R}}\right]_{C^{\alpha}} \frac{b_{4}}{R^{4}} \\
& \leq \\
& +\frac{b_{1}}{R^{2}} 2 R^{1-\alpha} \frac{C_{14}}{\delta}+\frac{1}{R} \frac{C_{16}}{\delta^{1+\alpha}}+4 \frac{b_{1}}{R^{2}} 2 R^{1-\alpha} b_{1} \frac{C_{13}}{R}+4 \frac{b_{2}}{R^{2}} 2 R^{1-\alpha} \frac{C_{13}}{R}+ \\
& +4 \frac{C_{15}}{\delta R} 2 R^{1-\alpha} \frac{b_{1}}{R}+6 \frac{b_{1}}{R^{2}} 2 R^{1-\alpha} \frac{b_{2}}{R} C_{3} \frac{\delta}{R}+6 \frac{b_{3}}{R^{2}} 2 R^{1-\alpha} \frac{1}{R} C_{3} \frac{\delta}{R}+ \\
& +6 \frac{C_{13}}{R} 2 R^{1-\alpha} \frac{b_{2}}{R^{2}}+4 \frac{b_{1}}{R^{2}} 2 R^{1-\alpha} \frac{b_{3}}{R^{2}} C_{2} \delta+4 \frac{b_{4}}{R^{3}} 2 R^{1-\alpha} \frac{1}{R} C_{2} \delta+ \\
& +\frac{b_{5}}{R^{4}} 2 R^{1-\alpha} \frac{1}{R} C_{1} \delta R+4 C_{3} \frac{\delta}{R} 2 R^{1-\alpha} \frac{b_{3}}{R^{3}}+\frac{b_{1}}{R^{2}} 2 R^{1-\alpha} \frac{b_{4}}{R^{3}} C_{1} \delta R+ \\
& +C_{2} \delta 2 R^{1-\alpha} \frac{b_{4}}{R^{4}} \leq C_{16} \frac{1}{R \delta^{1+\alpha}} \\
& +
\end{aligned}
$$

and the claim follows.

Lemma C.14 For $k_{a, R}$ and $g_{\delta}$ respectively as in (2.3) and (3.4) it holds that

$$
\left[(x, y) \mapsto(y-3 R) \frac{\partial^{4}}{\partial x^{4}} \frac{k_{a, R}-g_{\delta}}{3 R-k_{a, R}}\right]_{C^{\alpha}\left(\bar{\Omega}_{a, R}\right)} \leq \frac{C_{18}}{\delta^{1+\alpha}}
$$

Proof: The inequality

$$
[(x, y) \mapsto f(x) g(y)]_{C^{\alpha}[a, b]^{2}} \leq[f]_{C^{\alpha}[a, b]}\|g\|_{C^{0}[a, b]}+\|f\|_{C^{0}[a, b]}[g]_{C^{\alpha}[a, b]}
$$

implies that

$$
\left[(x, y) \mapsto(y-3 R) \frac{\partial^{4}}{\partial x^{4}} \frac{k_{a, R}-g_{\delta}}{3 R-k_{a, R}}\right]_{C^{\alpha}\left(\bar{\Omega}_{a, R}\right)} \leq
$$




$$
\begin{aligned}
\leq & 3 R^{1-\alpha}\left\|\frac{\partial^{4}}{\partial x^{4}} \frac{k_{a, R}-g_{\delta}}{3 R-k_{a, R}}\right\|_{C^{0}\left(-R x_{a}^{*}, R x_{a}^{*}\right)}+ \\
& +4 R\left[\frac{\partial^{4}}{\partial x^{4}} \frac{k_{a, R}-g_{\delta}}{3 R-k_{a, R}}\right]_{C^{\alpha}\left(-R x_{a}^{*}, R x_{a}^{*}\right)} \leq \ldots
\end{aligned}
$$

By Lemma C.11 and Lemma C.13 we get

$$
\cdots \leq 3 R^{1-\alpha} \frac{C_{15}}{\delta R}+4 R \frac{C_{17}}{R \delta^{1+\alpha}}<\frac{C_{18}}{\delta^{1+\alpha}} .
$$

The boundedness of $f_{a, R}$ in $C^{4, \alpha}$-norm follows directly from Proposition 3.12 and Lemmas C.9, C.11 and C.14.

\section{C.3 Partition of unity}

In this section we present a general result about partition of unity that we will use in the proof of Theorem 4.1.

\section{Lemma C.15 (Partition of unity with boundary)}

Let $\Omega \subset \mathbb{R}^{n}$ be a bounded domain and let $\left\{D_{j}\right\}_{j \in J} \subset \Omega$ be a finite open covering of $\Omega$ such that $\partial \Omega \subset \bigcup_{j \in J}\left(\partial D_{j} \cap \partial \Omega\right)^{\circ, \partial \Omega}$. For every $\delta>0$ there exist finitely many smooth functions $\psi_{i} \in C^{\infty}(\bar{\Omega}), i \in I$, such that:

1. $\psi_{i} \geq 0$ for all $i \in I$ and $\sum_{i \in I} \psi_{i}(x)=1$ for all $x \in \bar{\Omega}$;

2. for every $i \in I$ there exists $j=j(i)$ such that $\operatorname{supp}\left(\psi_{i}\right) \subset D_{j} \cup$ $\left(\partial D_{j} \cup \partial \Omega\right)^{\circ, \partial \Omega}$,

3. $\operatorname{diam}\left(\operatorname{supp}\left(\psi_{i}\right)\right) \leq \delta$ for all $i \in I$.

\section{Elliptic regularity and interpolation}

Elliptic regularity results for linear equations can be found in numerous places. However, if one goes beyond second order and if one needs to know how the constants depend on the domain there is no easy reference. For that reason we will collect such type of results in the present section. For the explicit dependence of these constants we will go back to the original source of Agmon, Douglis and Nirenberg ([2]). 
This section is organized as follows. First we recall some classical results and the Calderon-Zygmund inequality for $n=2$. Then we consider a strong and a weak formulation of problem (1.1). Finally we study three intermediate versions (between strong and weak) of problem (1.1).

Throughout this section the following condition will appear.

Condition D.1 The number $\alpha$ lies in $(0,1)$ and $\Omega$ is a bounded simply connected domain (open subset) in $\mathbb{R}^{2}$ satisfying the uniform $C^{4, \alpha}$ regularity condition with constant $M$.

\section{D.1 Classical results}

In this section we recall some results from [11]. For sake of brevity we do not give the most general statements.

Theorem D.2 [11, Th.9.13] Let $\Omega$ be a bounded domain in $\mathbb{R}^{n}$ satisfying the uniform $C^{1,1}$ regularity condition with constant $M$. Then it holds

$$
\|u\|_{W^{2,2}(\Omega)} \leq C\left(\|u\|_{L^{2}(\Omega)}+\|\Delta u\|_{L^{2}(\Omega)}\right)
$$

for every $u \in W^{2,2}(\Omega) \cap W_{0}^{1,2}(\Omega)$ and with $C=C\left(n, M, \rho_{\Omega}^{-1}, R_{\Omega}\right)$.

Remark D.3 The dependence of the constant can be deduced from the proof in [11, Th.9.13].

We will use the Calderon-Zygmund inequality for $n=2$. This inequality is usually proved by contradiction. Since we are interested in the dependence of the constant on the domain, we give here a direct proof.

Lemma D.4 Let $\Omega$ be a bounded domain in $\mathbb{R}^{2}$. Then there is $C=C\left(R_{\Omega}\right)$ such that

$$
\|u\|_{L^{2}(\Omega)} \leq C\|\Delta u\|_{L^{2}(\Omega)} \text { for every } u \in W^{2,2}(\Omega) \cap W_{0}^{1,2}(\Omega) .
$$

Proof: Let $u \in W^{2,2}(\Omega) \cap W_{0}^{1,2}(\Omega)$. For $n=2$ Aleksandrov's maximum principle ([11, Th.9.1]) implies that $\sup _{\Omega}|u| \leq C\|\Delta u\|_{L^{2}(\Omega)}$ for some $C=C\left(R_{\Omega}\right)$. Hence we find $\|u\|_{L^{2}(\Omega)} \leq C\|\Delta u\|_{L^{2}(\Omega)}|\Omega|^{\frac{1}{2}}$. 
Corollary D.5 Suppose $\Omega$ is a bounded domain in $\mathbb{R}^{2}$ satisfying the uniform $C^{1,1}$ regularity condition with constant $M$. Then there is $C=$ $C\left(M, \rho_{\Omega}^{-1}, R_{\Omega}\right)$ such that

$$
\|u\|_{W^{2,2}(\Omega)} \leq C\|\Delta u\|_{L^{2}(\Omega)} \text { for every } u \in W^{2,2}(\Omega) \cap W_{0}^{1,2}(\Omega) .
$$

Proof: The claim follows directly from Theorem D.2 and Lemma D.4.

\section{D.2 Regularity for strong solutions}

The classical regularity result that we like to recall in an explicit statement is the following.

Theorem D.6 Assume Condition D.1. For every $f \in L^{p}(\Omega)$ with $p \in$ $(1, \infty)$ there exists a unique solution $u \in W^{4, p}(\Omega) \cap W_{0}^{2, p}(\Omega)$ of (1.1).

Moreover the solution satisfies

$$
\frac{1}{2}\|f\|_{L^{p}(\Omega)} \leq\|u\|_{W^{4, p}(\Omega)} \leq C_{s}\|f\|_{L^{p}(\Omega)},
$$

with $C_{s}=C_{s}\left(p, p^{\prime}, M, \rho_{\Omega}^{-1}, R_{\Omega}\right)$ where $C_{s}$ satisfies the convention of Notation 1.9.

Before proving Theorem D.6 we present some estimates.

Lemma D.7 Let $\Omega$ be a bounded domain in $\mathbb{R}^{2}$ satisfying the uniform $C^{1,1}$ regularity condition with constant $M$ and let $p \in(1, \infty)$. Then there is $C=C\left(p, p^{\prime}, M, \rho_{\Omega}^{-1}, R_{\Omega}\right)$ such that:

$$
\|u\|_{L^{p}(\Omega)} \leq C\left\|\Delta^{2} u\right\|_{L^{p}(\Omega)} \text { for every } u \in W^{4, p}(\Omega) \cap W_{0}^{2, p}(\Omega) .
$$

Proof: Since $n=2$ we find by Sobolev inequalities that

$$
\|u\|_{L^{p}(\Omega)} \leq C_{1}\|u\|_{W^{2,2}(\Omega)} \text { and }\|u\|_{L^{p^{\prime}(\Omega)}} \leq C_{2}\|u\|_{W^{2,2}(\Omega)} .
$$

Notice that $C_{1}=C_{1}\left(p, M, \rho_{\Omega}^{-1}, R_{\Omega}\right)$ and $C_{2}=C_{2}\left(p^{\prime}, M, \rho_{\Omega}^{-1}, R_{\Omega}\right)$. Hence we have by Corollary D.5, by integrating by parts and by Hölder that

$$
\begin{aligned}
\|u\|_{L^{p^{\prime}}(\Omega)}\|u\|_{L^{p}(\Omega)} & \leq C_{1} C_{2}\|u\|_{W^{2,2}(\Omega)}^{2} \leq C_{3} \int_{\Omega}|\Delta u|^{2} d x= \\
& =C_{3} \int_{\Omega} u \Delta^{2} u d x \leq C_{3}\left\|\Delta^{2} u\right\|_{L^{p}(\Omega)}\|u\|_{L^{p^{\prime}}(\Omega)},
\end{aligned}
$$


with $C_{3}=C_{3}\left(p, p^{\prime}, M, \rho_{\Omega}^{-1}, R_{\Omega}\right)$. The claim follows.

Lemma D.8 Assume Condition D.1. Then for $1<p<\infty$ there exists $C=C\left(p, p^{\prime}, M, \rho_{\Omega}^{-1}, R_{\Omega}\right)$ such that

$$
\|u\|_{W^{4, p}(\Omega)} \leq C\left\|\Delta^{2} u\right\|_{L^{p}(\Omega)} \text { for every } u \in W^{4, p}(\Omega) \cap W_{0}^{2, p}(\Omega) .
$$

Remark D.9 Usually Lemma D.8 is proved by contradiction and this does not explain what the constant depends on. However by using Lemma D.7 we find the explicit quantities.

Proof: The result follows from [2, Th.15.2] and Lemma D.7. The proof of [2, Th.15.2] shows that the dependence of the constant is as given in the statement.

\section{Proof of Theorem D.6:}

- Uniqueness follows by a standard integration by parts. Indeed, if $\Delta^{2} u=0$ then

$$
\int_{\Omega}|\Delta u|^{2} d x=\int_{\Omega} u \Delta^{2} u d x=0
$$

and with the boundary condition one finds $u \equiv 0$.

- Estimate: By definition of the norm in $W^{4, p}(\Omega)$ one finds that $\frac{1}{2}\left\|\Delta^{2} u\right\|_{L^{p}(\Omega)} \leq\|u\|_{W^{4, p}(\Omega)}$. The other side of inequality (D.1) follows from Lemma D.8.

- Existence: For $f \in C^{\alpha}(\Omega)$ the existence of a solution $u \in C^{4, \alpha}(\Omega) \cap$ $C_{0}^{1}(\bar{\Omega})$ is given by [2, Th.12.7]. Such a solution satisfies (D.1), see [2, Th.9.3]. By an approximation the existence in $W^{4, p}(\Omega) \cap W_{0}^{2, p}(\Omega)$ follows.

Remark D.10 The hypothesis $\partial \Omega \in C^{4, \alpha}$ is needed in order to use Theorem 12.7 in [2]. For the rest of the paper it would be sufficient to assume $\partial \Omega \in C^{4}$.

For $1<p<\infty$ we formally define the operator $T_{4, p}$ by

$$
\begin{aligned}
D\left(T_{4, p}\right) & :=W^{4, p}(\Omega) \cap W_{0}^{2, p}(\Omega), \\
T_{4, p} u & :=\Delta^{2} u \text { for } u \in D\left(T_{4, p}\right) .
\end{aligned}
$$


The operator $T_{4, p}$ is the inverse of the solution operator.

The following result is a consequence of Theorem D.6.

Corollary D.11 Let $1<p<\infty$. Assuming Condition D.1 the operator $T_{4, p}$ defined in (D.2) gives an isomorphism from $W^{4, p}(\Omega) \cap W_{0}^{2, p}(\Omega)$ onto $L^{p}(\Omega)$. Moreover one has

$$
\frac{1}{C_{s}} \leq\left\|T_{4, p}\right\|_{\left(W^{4, p}(\Omega) \cap W_{0}^{2, p}(\Omega) \rightarrow L^{p}(\Omega)\right)} \leq 2
$$

where $C_{s}$ is the constant appearing in Theorem D.6.

\section{D.3 Regularity for weak solutions}

In the following section we give the explicit definition of what we will call a weak solution for problem (1.1) and we recall the classical regularity result in this setting.

Definition D.12 Let $p \in(1, \infty)$ and $F \in\left(W^{4, p^{\prime}}(\Omega) \cap W_{0}^{2, p^{\prime}}(\Omega)\right)^{\prime}$. We call $u \in L^{p}(\Omega)$ a weak solution of problem (1.1) with right hand side $F$ if the following holds

$$
\int_{\Omega} u(x) \Delta^{2} v(x) d x=F(v) \text { for every } v \in W^{4, p^{\prime}}(\Omega) \cap W_{0}^{2, p^{\prime}}(\Omega) .
$$

Theorem D.13 Assume Condition D.1 and let $p \in(1, \infty)$. Then for every $F \in\left(W^{4, p^{\prime}}(\Omega) \cap W_{0}^{2, p^{\prime}}(\Omega)\right)^{\prime}$ there exists a unique u weak solution of problem (1.1) with right hand side F. Moreover $u$ satisfies

$$
\frac{1}{2}\|F\|_{\left(W^{4, p^{\prime}}(\Omega) \cap W_{0}^{2, p^{\prime}}(\Omega)\right)^{\prime}} \leq\|u\|_{L^{p}(\Omega)} \leq C_{w}\|F\|_{\left(W^{4, p^{\prime}}(\Omega) \cap W_{0}^{2, p^{\prime}}(\Omega)\right)^{\prime}},
$$

with $C_{w}=C_{w}\left(p, p^{\prime}, M, \rho_{\Omega}^{-1}, R_{\Omega}\right)$.

Proof: Let $i_{p}$ be the canonical isometry $L^{p}(\Omega) \rightarrow\left(L^{p^{\prime}}(\Omega)\right)^{\prime}$, that is, $i_{p}(u)(v)=\int_{\Omega} u(x) v(x) d x$ for every $v \in L^{p^{\prime}}(\Omega)$. 
Existence of $u$ follows by a duality argument. Indeed, by Corollary D.11 we may define

$$
U(f):=F\left(T_{4, p^{\prime}}^{-1}(f)\right) \text { for every } f \in L^{p^{\prime}}(\Omega) .
$$

The solution $u$ is given by $u:=i_{p}^{-1}(U)$. Uniqueness and the estimate follow from Corollary D.11.

For $1<p<\infty$ let us formally define the operator $T_{0, p}$ by

$$
\begin{aligned}
D\left(T_{0, p}\right) & :=L^{p}(\Omega), \\
\left(T_{0, p}(u)\right)(v) & :=i_{p}(u)\left(T_{4, p^{\prime}}(v)\right) \text { for every } v \in W^{4, p^{\prime}}(\Omega) \cap W_{0}^{2, p^{\prime}}(\Omega) .
\end{aligned}
$$

By Theorem D.13 it follows:

Corollary D.14 Let $1<p<\infty$ and assume Condition D.1 is satisfied. Then the operator $T_{0, p}$ defined in (D.3) gives an isomorphism from $L^{p}(\Omega)$ onto $\left(W^{4, p^{\prime}}(\Omega) \cap W_{0}^{2, p^{\prime}}(\Omega)\right)^{\prime}$. Moreover one has

$$
\frac{1}{C_{w}} \leq\left\|T_{0, p}\right\|_{\left(L^{p}(\Omega) \rightarrow\left(W^{4, p^{\prime}}(\Omega) \cap W_{0}^{2, p^{\prime}}(\Omega)\right)^{\prime}\right)} \leq 2,
$$

where $C_{w}$ is the constant appearing in Theorem D.13.

\section{D.4 Regularity between weak and strong}

In the following section we consider via interpolation solutions between the 'strong' and the 'weak' ones defined in the previous sections.

We first give the three intermediate notions of solution.

Definition D.15 Let $p \in(1, \infty)$.

1. Let $F \in\left(W_{0}^{1, p^{\prime}}(\Omega)\right)^{\prime}$. We say that $u \in W^{3, p}(\Omega) \cap W_{0}^{2, p}(\Omega)$ is a "one-quarter weak solution" of problem (1.1) with right hand side $F$ if it satisfies

$$
-\int_{\Omega}(\nabla \Delta u(x)) .(\nabla v(x)) d x=F(v) \text { for every } v \in W_{0}^{1, p^{\prime}}(\Omega) .
$$


2. Let $F \in\left(W_{0}^{2, p^{\prime}}(\Omega)\right)^{\prime}$. We say that $u \in W_{0}^{2, p}(\Omega)$ is a "one-half weak solution" of problem (1.1) with right hand side $F$ if it satisfies

$$
\int_{\Omega}(\Delta u(x))(\Delta v(x)) d x=F(v) \text { for every } v \in W_{0}^{2, p^{\prime}}(\Omega) .
$$

3. Let $F \in\left(W^{3, p^{\prime}}(\Omega) \cap W_{0}^{2, p^{\prime}}(\Omega)\right)^{\prime}$. We say that $u \in W_{0}^{1, p}(\Omega)$ is a "three-quarter weak solution" of problem (1.1) with right hand side $F$ if it satisfies for every $v \in W^{3, p^{\prime}}(\Omega) \cap W_{0}^{2, p^{\prime}}(\Omega)$

$$
-\int_{\Omega}(\nabla u(x)) \cdot(\nabla \Delta v(x)) d x=F(v) .
$$

Theorem D.16 Assume Condition D.1 and let $1<p<\infty$.

1. Then for every $F \in\left(W_{0}^{1, p^{\prime}}(\Omega)\right)^{\prime}$ there exists a unique u "onequarter weak solution" of problem (1.1) with right hand side F.

Moreover u satisfies

$$
\frac{1}{C_{1}}\|F\|_{\left(W_{0}^{1, p^{\prime}}(\Omega)\right)^{\prime}} \leq\|u\|_{W^{3, p}(\Omega)} \leq C_{1}\|F\|_{\left(W_{0}^{1, p^{\prime}}(\Omega)\right)^{\prime}},
$$

with $C_{1}=C_{1}\left(p, p^{\prime}, M, \rho_{\Omega}^{-1}, R_{\Omega}\right)$.

2. Then for every $F \in\left(W_{0}^{2, p^{\prime}}(\Omega)\right)^{\prime}$ there exists a unique u "one-half weak solution" of problem (1.1) with right hand side $F$.

Moreover u satisfies

$$
\frac{1}{C_{2}}\|F\|_{\left(W_{0}^{2, p^{\prime}}(\Omega)\right)^{\prime}} \leq\|u\|_{W^{2, p}(\Omega)} \leq C_{2}\|F\|_{\left(W_{0}^{2, p^{\prime}}(\Omega)\right)^{\prime}},
$$

with $C_{2}=C_{2}\left(p, p^{\prime}, M, \rho_{\Omega}^{-1}, R_{\Omega}\right)$.

3. Then for every $F \in\left(W^{3, p^{\prime}}(\Omega) \cap W_{0}^{2, p^{\prime}}(\Omega)\right)^{\prime}$ there exists a unique $u$ "three-quarter weak solution" of problem (1.1) with right hand side F. 
Moreover u satisfies

$$
\begin{aligned}
& \frac{1}{C_{3}}\|F\|_{\left(W^{3, p^{\prime}}(\Omega) \cap W_{0}^{2, p^{\prime}}(\Omega)\right)^{\prime}} \leq\|u\|_{W^{1, p}(\Omega)} \leq C_{3}\|F\|_{\left(W^{3, p^{\prime}}(\Omega) \cap W_{0}^{2, p^{\prime}}(\Omega)\right)^{\prime}} \\
& \text { with } C_{3}=C_{3}\left(p, p^{\prime}, M, \rho_{\Omega}^{-1}, R_{\Omega}\right) .
\end{aligned}
$$

Remark D.17 Theorem D.16 part 2 has been studied in [23, Chap.7].

Our aim in giving the proof of Theorem D.16 is to show how the constants in the estimates depend on the domain. We proceed through interpolation: $[\cdot, \cdot]_{\theta}$ denotes the complex interpolation with parameter $\theta \in(0,1)$.

For sake of conciseness we use the following notation:

$$
\begin{aligned}
& A_{0, p}:=L^{p}(\Omega) \quad A_{4, p}:=W^{4, p}(\Omega) \cap W_{0}^{2, p}(\Omega), \\
& B_{0, p}:=\left(A_{4, p^{\prime}}\right)^{\prime} \quad B_{4, p}:=L^{p}(\Omega)\left(\cong\left(A_{0, p^{\prime}}\right)^{\prime}\right),
\end{aligned}
$$

and for $\theta \in(0,1)$

$$
A_{4 \theta, p}:=\left[A_{0, p}, A_{4, p}\right]_{\theta} \text { and } B_{4 \theta, p}:=\left[B_{0, p}, B_{4, p}\right]_{\theta} .
$$

With this notation we have $T_{0, p}: A_{0, p} \rightarrow B_{0, p}$ and $T_{4, p}: A_{4, p} \rightarrow B_{4, p}$, where $T_{0, p}$ is defined in (D.3) and $T_{4, p}$ is defined in (D.2).

Lemma D.18 Assume Condition D. 1 and let $1<p<\infty$. The operator $T_{4, p}$ is a restriction of $T_{0, p}$ to $A_{4, p}$ in the sense that for every $u \in A_{4, p}$

$$
T_{0, p}(u) \in\left(B_{4, p^{\prime}}\right)^{\prime} \text { and } T_{0, p}(u)=i_{p}\left(T_{4, p}(u)\right) .
$$

Proof: Let $u \in A_{4, p}$. For every $v \in A_{4, p^{\prime}}$ we have

$$
\left(T_{0, p}(u)\right)(v)=\int_{\Omega} u \Delta^{2} v d x=\int_{\Omega} v \Delta^{2} u d x=\int_{\Omega} v T_{4, p}(u) d x .
$$

The claim follows.

As a consequence of Lemma D.18 in the following lemma we find via interpolation a family of isomorphisms which are extensions of $T_{4, p}$ and restrictions of $T_{0, p}$. 
Lemma D.19 Assume Condition D.1 and let $\theta \in(0,1)$. Consider the operator $T_{4 \theta, p}$ such that $D\left(T_{4 \theta, p}\right):=A_{4 \theta, p}$ and $T_{4 \theta, p}(u):=T_{0, p}(u)$ for $u \in D\left(T_{4 \theta, p}\right)$ and $1<p<\infty$.

Then $T_{4 \theta, p}$ is an isomorphism from $A_{4 \theta, p}$ onto $B_{4 \theta, p}$ and moreover

$$
\frac{1}{\max \left\{C_{s}, C_{w}\right\}} \leq\left\|T_{4 \theta, p}\right\|_{\left(A_{4 \theta, p} \rightarrow B_{4 \theta, p}\right)} \leq 2,
$$

where $C_{s}$ and $C_{w}$ are the constants appearing in Theorems D.6 and D.13 respectively.

Proof: The claim follows from Corollaries D.11 and D.14 since the complex interpolation functor is exact and of type $\theta$ ([25, Th.1.9.3a]).

Remark D.20 Notice that (D.4) implies that for every $u \in A_{4 \theta, p}$ it holds

$$
\frac{1}{2}\left\|T_{4 \theta, p}(u)\right\|_{B_{4 \theta, p}} \leq\|u\|_{A_{4 \theta, p}} \leq \max \left\{C_{s}, C_{w}\right\}\left\|T_{4 \theta, p}(u)\right\|_{B_{4 \theta, p}} .
$$

In the following we consider the operators $T_{1, p}, T_{2, p}$ and $T_{3, p}$; i.e. the operators $T_{4 \theta_{i}, p}$ defined in Lemma D.19 with $\theta_{i}=\frac{1}{4} i$ and $i=1,2,3$. Notice that the solution operator for the "three-quarter weak solution" of problem (1.1) is the inverse of $T_{1, p}$. Analogously the solution operator for the "one-half weak solution" of problem (1.1) is the inverse of $T_{2, p}$ and the solution operator for the "one-quarter weak solution" of problem (1.1) is the inverse of $T_{3, p}$.

For these operators we have that

$$
A_{i, p}=W^{i, p}(\Omega) \cap W_{0}^{\min \{i, 2\}, p}(\Omega) \text { with equivalent norms, }
$$

where $A_{i, p}=D\left(T_{i, p}\right)$. Identity (D.5) can be found in Triebel for $C^{\infty}$ domains. We first show that in order (D.5) to hold it is sufficient that $\partial \Omega \in C^{4, \alpha}$. Furthermore we give the dependence on the domain of the constants $D_{1, p, i}$ and $D_{2, p, i}$ that appear in

$$
\begin{aligned}
& D_{1, p, i}\|u\|_{W^{i, p}(\Omega) \cap W_{0}^{\min \{i, 2\}, p}(\Omega)} \leq\|u\|_{A_{i, p}} \leq D_{2, p, i}\|u\|_{W^{i, p}(\Omega) \cap W_{0}^{\min \{i, 2\}, p}(\Omega)}, \\
& \text { for } u \in W^{i, p}(\Omega) \cap W_{0}^{\min \{i, 2\}, p}(\Omega) .
\end{aligned}
$$

We first recall a classical result from [25]. 
Proposition D.21 [25, Th.4.3.3] Let $B$ denote the unit ball in $\mathbb{R}^{n}$. Then for $i=1,2,3$ and $1<p<\infty$ one has

$$
\left[L^{p}(B), W^{4, p}(B) \cap W_{0}^{2, p}(B)\right]_{\frac{1}{4} i}=W^{i, p}(B) \cap W_{0}^{\min \{i, 2\}, p}(B)
$$

as Banach spaces (equivalent norms). Hence there exist constants $C_{1, p, i}$ and $C_{2, p, i}$ such that for every $u \in W^{i, p}(B) \cap W_{0}^{\min \{i, 2\}, p}(B)$ one has

$$
C_{1, p, i}\|u\|_{W^{i, p}(B)} \leq\|u\|_{\left[L^{p}(B), W^{4, p}(B) \cap W_{0}^{2, p}(B)\right]_{\frac{1}{4} i}} \leq C_{2, p, i}\|u\|_{W^{i, p}(B)} .
$$

Theorem D.22 Let assume Condition D.1. Then for $1<p<\infty$ and $i=1,2,3$ it holds

$$
\left[A_{0, p}, A_{4, p}\right]_{\frac{1}{4} i}=W^{i, p}(\Omega) \cap W_{0}^{\min \{i, 2\}, p}(\Omega) .
$$

Hence there exist constants $D_{1, p, i}$ and $D_{2, p, i}$ such that for every $u \in$ $W^{i, p}(\Omega) \cap W_{0}^{\min \{i, 2\}, p}(\Omega)$ one has

$$
D_{1, p, i}\|u\|_{W^{i, p}(\Omega)} \leq\|u\|_{\left[A_{0, p}, A_{4, p}\right]_{\frac{1}{4} i}} \leq D_{2, p, i}\|u\|_{W^{i, p}(\Omega)},
$$

with $D_{j, p, i}=D_{j, p, i}\left(p, M, \rho_{\Omega}^{-1}, R_{\Omega}\right)$ for $j=1,2$.

Proof: Let $S$ denote a $C^{4, \alpha}$ transformation from $\bar{\Omega}$ onto $\bar{B}$.

Considering the operator

$$
E_{p}: L^{p}(\Omega) \rightarrow L^{p}(B) \text { such that } E_{p}(f):=f \circ S^{-1},
$$

one finds that the following properties hold:

- $E_{p}$ is an isomorphism;

- for $i=1, \ldots, 4$ the restriction of $E_{p}$ to $W^{i, p}(\Omega) \cap W_{0}^{\min \{i, 2\}, p}(\Omega)$ is an isomorphism onto $W^{i, p}(B) \cap W_{0}^{\min \{i, 2\}, p}(B)$;

- there are constants $\bar{C}_{1, p}$ and $\bar{C}_{2, p}$ such that

$$
\bar{C}_{1, p}\left\|E_{p}(u)\right\|_{W^{i, p}(B)} \leq\|u\|_{W^{i, p}(\Omega)} \leq \bar{C}_{2, p}\left\|E_{p}(u)\right\|_{W^{i, p}(B)},
$$

for every $i=0,1, \ldots, 4$ and $u \in W^{i, p}(\Omega) \cap W_{0}^{\min \{i, 2\}, p}(\Omega)$. Furthermore the constants $\bar{C}_{1, p}$ and $\bar{C}_{2, p}$ depend only on $p, R_{\Omega}, \rho_{\Omega}^{-1}$ and the $M$ of Condition D.1. 
For $\theta \in(0,1)$ the operator $E_{p}$ induces isomorphisms

$$
E_{p, \theta}: A_{4 \theta, p} \rightarrow\left[L^{p}(B), W^{4, p}(B) \cap W_{0}^{2, p}(B)\right]_{\theta},
$$

and since the complex interpolation functor is exact ([25, Th.1.9.3a]) one has

$$
\begin{aligned}
& \bar{C}_{1, p}\left\|E_{p, \theta}(u)\right\|_{\left[L^{p}(B), W^{4, p}(B) \cap W_{0}^{2, p}(B)\right]_{\theta}} \\
\leq & \|u\|_{A_{4 \theta, p}} \leq \bar{C}_{2, p}\left\|E_{p, \theta}(u)\right\|_{\left[L^{p}(B), W^{4, p}(B) \cap W_{0}^{2, p}(B)\right]_{\theta}},
\end{aligned}
$$

(See Theorem 1.2.4 in [25]).

Hence, by (D.7) and Proposition D.21, we have that

$$
\begin{aligned}
& A_{i, p}=\left[L^{p}(\Omega), W^{4, p}(\Omega) \cap W_{0}^{2, p}(\Omega)\right]_{\frac{1}{4} i} \\
& =\left(E_{p, \frac{1}{4} i}\right)^{-1}\left(\left[L^{p}(B), W^{4, p}(B) \cap W_{0}^{2, p}(B)\right]_{\frac{1}{4} i}\right) \\
& =\left(E_{p, \frac{1}{4} i}\right)^{-1}\left(W^{i, p}(B) \cap W_{0}^{\min \{i, 2\}, p}(B)\right)=W^{i, p}(\Omega) \cap W_{0}^{\min \{i, 2\}, p}(\Omega) .
\end{aligned}
$$

Furthermore we explicitly find the constants that give the equivalence of the norms. Indeed from (D.6), (D.7) and Proposition D.21 it follows

$$
\begin{aligned}
\|u\|_{A_{i, p}} & \leq \bar{C}_{2, p}\left\|E_{p, \frac{1}{4} i}(u)\right\|_{\left[L^{p}(B), W^{4, p}(B) \cap W_{0}^{2, p}(B)\right]_{\frac{1}{4} i}} \\
& \leq \bar{C}_{2, p} C_{2, p, i}\left\|E_{p, \frac{1}{4} i}(u)\right\|_{W^{i, p}(B)} \leq \frac{\bar{C}_{2, p}}{\bar{C}_{1, p}} C_{2, p, i}\|u\|_{W^{i, p}(\Omega)},
\end{aligned}
$$

and

$$
\begin{aligned}
& \|u\|_{W^{i, p}(\Omega)} \leq \bar{C}_{2, p}\left\|E_{p, \frac{1}{4} i}(u)\right\|_{W^{i, p}(B)} \\
& \leq \frac{\bar{C}_{2, p}}{C_{1, p, i}}\left\|E_{p, \frac{1}{4} i}(u)\right\|_{\left[L^{p}(B), W^{4, p}(B) \cap W_{0}^{2, p}(B)\right]_{\frac{1}{4} i}} \leq \frac{\bar{C}_{2, p}}{\bar{C}_{1, p}} \frac{1}{C_{1, p, i}}\|u\|_{A_{i, p}} .
\end{aligned}
$$

Remark D.23 The existence of the $C^{4, \alpha}$ transformation from $\bar{\Omega}$ onto $\bar{B}$ depends upon the regularity of $\Omega$ and the fact that $\Omega$ is simply connected. This technical assumption can be removed. 
Corollary D.24 Let assume Condition D.1. Then for $1<p<\infty$ and $i=1,2,3$ it holds

$$
\left[B_{0, p}, B_{4, p}\right]_{\frac{1}{4} i}=\left(W^{4-i, p^{\prime}}(\Omega) \cap W_{0}^{\min \{4-i, 2\}, p^{\prime}}(\Omega)\right)^{\prime} .
$$

Moreover there exist constants $\bar{D}_{j, p, i}=\bar{D}_{j, p, i}\left(p^{\prime}, M, \rho_{\Omega}^{-1}, R_{\Omega}\right)$ for $j=1,2$ such that

$$
\begin{aligned}
& \bar{D}_{1, p, i}\|u\|_{\left(W^{4-i, p^{\prime}}(\Omega) \cap W_{0}^{\min \{4-i, 2\}, p^{\prime}}(\Omega)\right)^{\prime}} \\
\leq & \|u\|_{\left[B_{0, p}, B_{4, p}\right]_{\frac{1}{4}}} \leq \bar{D}_{2, p, i}\|u\|_{\left(W^{4-i, p^{\prime}}(\Omega) \cap W_{0}^{\min \{4-i, 2\}, p^{\prime}}(\Omega)\right)^{\prime}},
\end{aligned}
$$

holds for every $u \in\left(W^{4-i, p^{\prime}}(\Omega) \cap W_{0}^{\min \{4-i, 2\}, p^{\prime}}(\Omega)\right)^{\prime}$.

Proof: The result follows from Theorem D.22 through duality results for complex interpolation spaces ([25, Th.1.11.3]).

Corollary D.25 Assume Condition D.1 and let $1<p<\infty$.

Then for $i=1,2,3$ there exist isomorphisms

$$
T_{i, p}: W^{i, p}(\Omega) \cap W_{0}^{\min \{i, 2\}, p}(\Omega) \rightarrow\left(W^{4-i, p^{\prime}}(\Omega) \cap W_{0}^{\min \{4-i, 2\}, p^{\prime}}(\Omega)\right)^{\prime},
$$

which are restrictions of $T_{0, p}$ and extensions of $T_{4, p}$.

Moreover there exists constants $C_{i}=C_{i}\left(p, p^{\prime}, M, \rho_{\Omega}^{-1}, R_{\Omega}\right)$ such that for every $u \in W^{i, p}(\Omega) \cap W_{0}^{\min \{i, 2\}, p}(\Omega)$ it holds

$$
\begin{aligned}
& \frac{1}{C_{i}}\left\|T_{i, p}(u)\right\|_{\left(W^{4-i, p^{\prime}}(\Omega) \cap W_{0}^{\min \{4-i, 2\}, p^{\prime}}(\Omega)\right)^{\prime}} \\
\leq & \|u\|_{W^{i, p}(\Omega)} \leq C_{i}\left\|T_{i, p}(u)\right\|_{\left(W^{4-i, p^{\prime}}(\Omega) \cap W_{0}^{\min \{4-i, 2\}, p^{\prime}}(\Omega)\right)^{\prime} .}
\end{aligned}
$$

Proof: The result follows from Lemma D.19, Theorem D.22 and Corollary D.24.

Theorem D.16 follows directly from the previous corollary. 


\section{References}

[1] R.A. Adams, J.J.F. Fournier, Sobolev spaces, Second Edition, Academic Press, 2003.

[2] S. Agmon, A. Douglis, L. Nirenberg, Estimates Near the Boundary for Solutions of Elliptic Partial Differential Equations Satisfying General Boundary Conditions, Comm. Pure App. Math. Vol.XII (1959), 623-727.

[3] T. Boggio, Sulle funzioni di Green d'ordine m, Rend. Circ. Mat. Palermo 20 (1905), 97-135.

[4] H. Brezis, Analyse Fonctionnelle, Masson, Paris, 1983.

[5] R. Courant, Dirichlet's Principle, Conformal Mapping, and Minimal Surfaces, Interscience, New York, 1970.

[6] Ph. Clément, G. Sweers, Uniform anti-maximum principle for polyharmonic boundary value problems, Proc. Amer. Math. Soc., 129 n.2 (2000), 467-474.

[7] A. Dall'Acqua, G. Sweers, The clamped plate equation for the Limaçon, Annali di Matematica Pura ed Applicata 184 (2005), 361374.

[8] A. Dall'Acqua, G. Sweers, Estimates for Green Function and the Poisson Kernels of higher order Dirichlet boundary value problems, J. Differential Equations 205 (2004), 466-487.

[9] R. Denk, M. Hieber, J. Prüss, $\mathcal{R}$-boundedness, Fourier multipliers and problems of elliptic and parabolic type, Mem. Amer. Math. Soc. 166 (2003), no. 788.

[10] R.J. Duffin, On a question of Hadamard concerning superbiharmonic functions, J. Math. Physics 27 (1949), 253-258.

[11] D. Gilbarg, N. Trudinger, Elliptic partial Differential Equations of Second Order, Springer-Verlag, 1977.

[12] H.-Ch. Grunau, G. Sweers, Positivity for perturbation of polyharmonic operators, Math. Nachr. 179 (1996), 89-102. 
[13] H.-Ch. Grunau, G. Sweers, Positivity for equations involving polyharmonic operators with Dirichlet boundary conditions, Math. Ann. 307 (1997), 589-626.

[14] H.-Ch. Grunau, G. Sweers, Classical solutions for some higher order semilinear elliptic equations under weak growth conditions, Nonlinear Analysis 28 n.5 (1997), 799-807.

[15] H.-Ch. Grunau, G. Sweers, The maximum principle and positive principal eigenfunctions for polyharmonic equations, in Reaction Diffusion Systems, Marcel Dekker Inc., New York 1997, 163-182.

[16] H.-Ch. Grunau, G. Sweers, The role of positive boundary data in the generalized clamped plate equation, ZAMP 49 (1998), 420-435.

[17] J. Hadamard, Memoire sur le probleme d'analyse relatif a l'equilibrie des plaques elastiques encastrees, in: Euvres de Jacques Hadamard, Tomes II, Éditions du Centre National de la Recherche Scientifique, Paris 1968, 515-641, reprint of: Mèmoire couronne par l'Acadèmie des Sciences (Prix Vaillant), Mèm. Sav. Etrang. 33 (1907).

[18] J.P. Krasovskiı̌, Isolation of Singularities of the Green Function, Math. USSR - Izvestija 1 No.5 (1967), 935-966.

[19] Z. Nehari, On the biharmonic Green's functions, Studies in mathematics and mechanics presented to Richard von Mises, Academic Press Inc., New York (1954), 111-117.

[20] M. Nicolescu, Les fonctions polyharmoniques, Hermann, Paris, 1936.

[21] Ch. Pommerenke, Boundary behavior of conformal maps, SpringerVerlag, 1992.

[22] E. Sassone, Positivity for polyharmonic problems on domains close to a disk, to appear in Annali di Matematica Pura ed Applicata.

[23] Ch.G. Simader, On Dirichlet's boundary value problem, Lecture Notes in Mathematics 268, Springer, (1972). 
[24] G. Sweers, Positivity for a strongly coupled elliptic system by Green function estimates, J. Geometric Analysis, 4 (1994), 121-142.

[25] H. Triebel, Interpolation Theory, Function Spaces, Differential Operators, North Holland publishing Company, 1978.

Anna Dall'Acqua

Mathematisches Institut

Ludwig-Maximilians-Universität München

Theresienstraße 39

80333 München, Germany

dallacqu@mathematik. uni-muenchen. de

Christian Meister

Alemannenstraße 20

88161 Lindenberg, Germany

chm. ecd@web. de

Guido Sweers

Delft Institute of Applied Mathematics

Delft University of Technology

PObox 5031

2600 GA Delft, The Netherlands

g.h.sweersatudelft.nl 